\title{
Three essays on the labour market implications of youth training programs and institutional changes in professional sports
}

\author{
by \\ Mihailo Radoman \\ Doctor of Philosophy \\ in \\ Economics \\ Carleton University \\ Ottawa,Ontario,Canada \\ (c) 2015 \\ Mihailo Radoman
}

A thesis submitted to the Faculty of Graduate and Postdoctoral Affairs in partial fulfillmen of the requirements for the degree of 


\section{Abstract}

The firs chapter of the thesis examines the impact of youth training programs

on career and spell duration of professional athletes. A unique data set of post-war English trained soccer players is used to study the impact of the youth training program they attended on their career and spell duration. The results indicate that the duration patterns of players are dependent on the youth academy they attended. The spell analysis outlines the nature of the competitive environment between smaller and larger clubs. Finally, the results of career and spell duration analyses addressed unobserved heterogeneity, allowed for nonlinearity of covariates using the cubic spline methodology, and were tested for endogeneity bias using a split sample test.

The second chapter analyzes certain labour market implications of institutional changes in professional sports. The study examines the impact of the Bosman ruling on the competitive nature of new entrants to the English Premier League. Relevant labour economics literature would predict that post-Bosman entrants will be more productive and consequently have a higher probability of securing a first-tea spot in top European leagues. To test these predictions, proprietary data was collected on all players that entered the English Premier League in four-year windows around the Bosman ruling. Regression Discontinuity Design displayed evident discontinuity in certain 
proxies for player productivity around the ruling. In addition, strong and robust empirical support for the motivating predictions is established through the application of survival analysis.

The third chapter utilizes the data sets from the firs two chapters and analyzes internal promotion versus external hiring in the English Premier League. Robust results are established in support of more reputable youth programs providing more first-tea opportunities through internal sources, consistently even in the aftermath of a major institutional change. Foreign sourced players become more prevalent in the league after the Bosman ruling, and their probability of selection is positively correlated with club stature. A separate survival analysis validates prior results in terms of youth training reputation of certain clubs, and establishes a presence of heterogeneity at youth club level that signals differences in player career prospects generated by their youth training. 


\section{Acknowledgments}

There are many people that deserve recognition and my sincere gratitude in making this thesis possible, so I must apologize to the ones that are not mentioned here. I have to start with my supervisor, Dr. Marcel Voia, without whom this project would be only a distant dream. His timely motivation and guidance throughout the process are greatly appreciated, and I owe a large debt to him in giving me invaluable educational and personal advice at times when I stumbled and questioned myself on this path.

I am also deeply indebted to Dr. Leo Michelis and Dr. Ingrid Bryan from Ryerson University who were most inspirational and influentia in my pursuit of a postgraduate education in economics. I am very thankful to my thesis committee members, Dr. Christopher Worswick from Carleton University and Dr. Louis-Philippe Morin from the University of Ottawa, who provided me with valuable input in improving the quality of my papers. I would also like to thank Dr. Patrick Coe from Carleton University who attended most of my workshops and provided valuable suggestions, and Marge Brooks for helping me with numerous issues along the way.

Last, but definitel not least, I would like to thank my loving family for supporting me on this journey. My parents provided me with the opportunity and all the necessary support to pursue my post-secondary education, and I only wish my father, Velimir, was still alive to share this moment with me. My wife, Vanja Radoman, deserves the most credit in holding down the "home-front" and allowing me to take the necessary time to complete this thesis. Her love and understanding made this dream 
a reality. All three of my daughters were born during my doctoral studies at Carleton University and they crystallized my goals and objectives in life, and served as the greatest inspiration in completing this thesis. Jovana, Lana, and Nina, I hope that this will one day inspire and motivate you in attaining greater things in life, much like your existence and love motivated me. 


\section{Table of Contents}

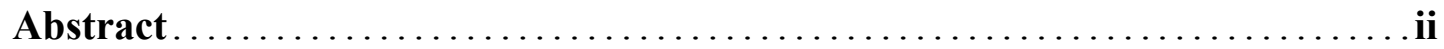

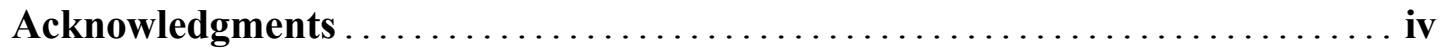

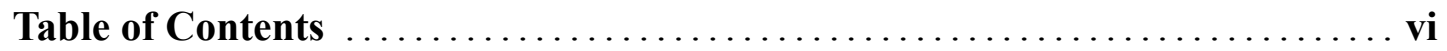

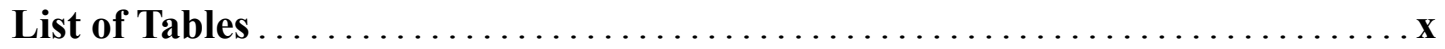

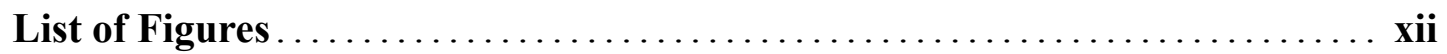

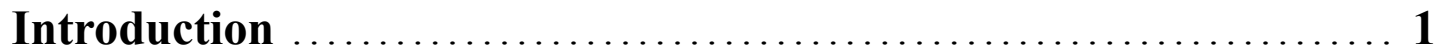

1 Youth Training Programs and their Impact on Career and Spell Duration of Professional Soccer Players ................. 5

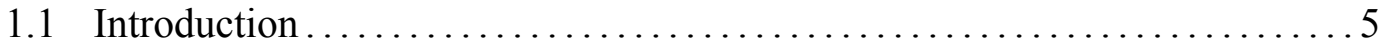

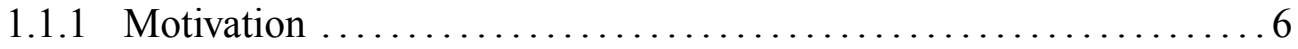

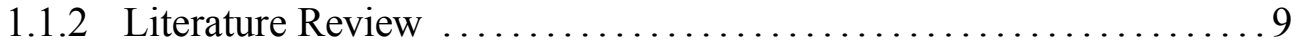

1.1.3 Short Summary of the Chapter and its Contribution $\ldots \ldots \ldots \ldots 10$

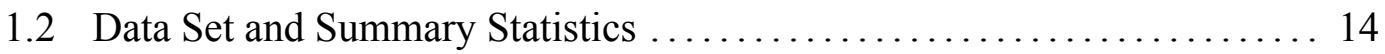

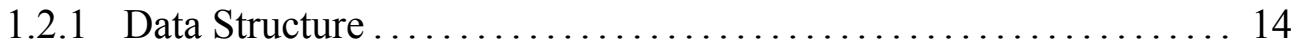




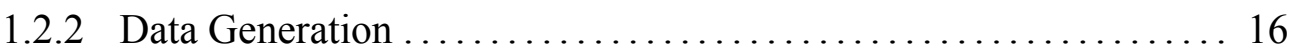

1.2.3 Broader Applicability ............................ 18

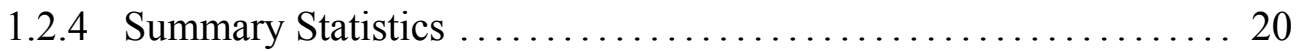

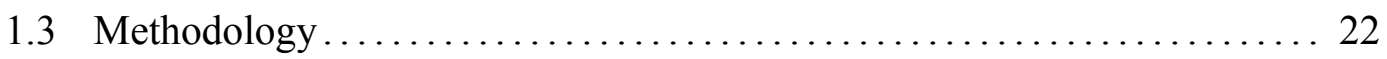

1.3.1 Functional Form of Covariates $\ldots \ldots \ldots \ldots \ldots \ldots \ldots \ldots \ldots \ldots$

1.3.2 State Dependence versus Unobserved Heterogeneity ......... 26

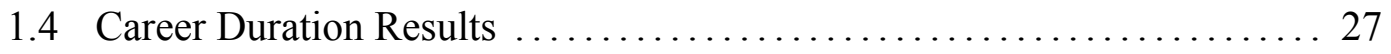

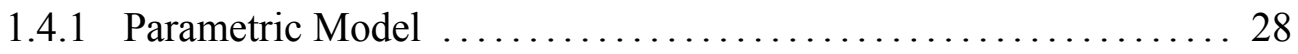

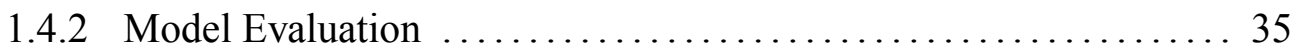

1.5 Home-Team Spell Duration Results ....................... 37

1.5.1 Semiparametric Model ........................ 38

1.6 Endogeneity Testing ............................... 42

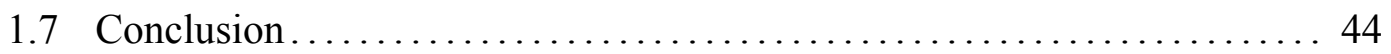

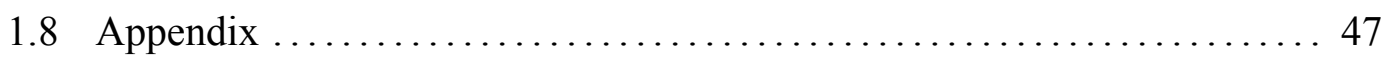

1.8.1 Web Sites Used in Data Collection .................. 47

1.8.2 Parametric Models............................ 47

1.8.3 Semiparametric Models ....................... 48

1.8.4 Vuong Test for Comparing Two Non-Nested Models ......... 50

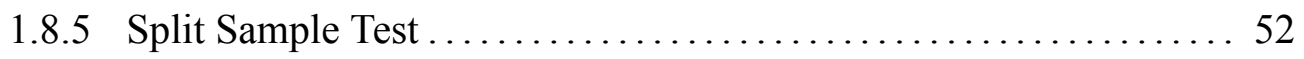

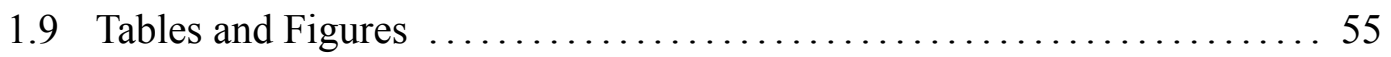

2 Labor market implications of institutional changes in European soccer: The Bosman ruling and its effect on productivity and career duration of players ................ 69 


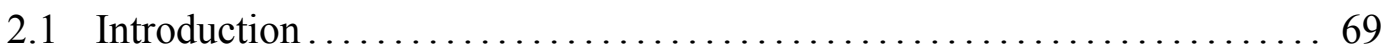

2.2 Motivating Theories and Review of Literature $\ldots \ldots \ldots \ldots \ldots \ldots \ldots$

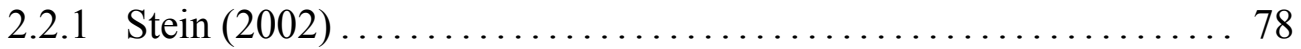

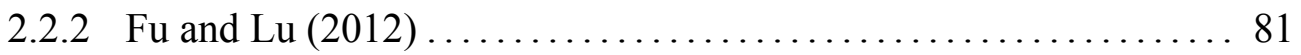

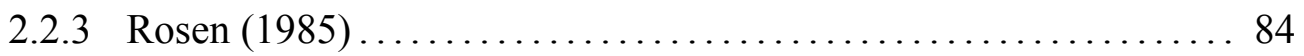

2.2.4 Other Relevant Discussions $\ldots \ldots \ldots \ldots \ldots \ldots \ldots \ldots \ldots \ldots \ldots$

2.3 Data Set and Summary Statistics $\ldots \ldots \ldots \ldots \ldots \ldots \ldots \ldots \ldots$

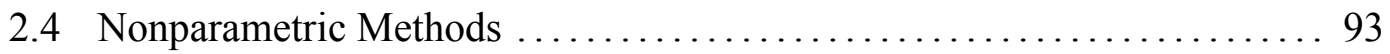

2.4.1 Regression Discontinuity (RD) Design $\ldots \ldots \ldots \ldots \ldots \ldots \ldots$

2.4.2 Nonparametric Survival Analysis .................... 97

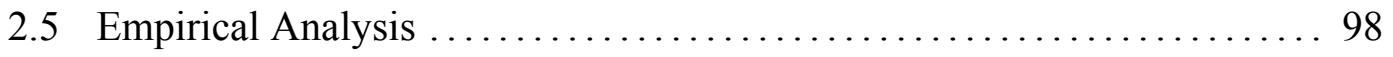

2.5.1 Results for Pre-Bosman entrants .................. 102

2.5.2 Results for Post-Bosman entrants ................ 106

2.5.3 Comparative Results .......................... 109

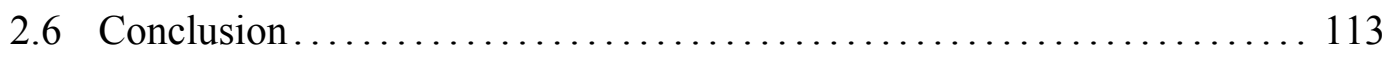

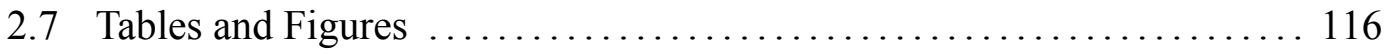

\section{Internal Promotion in Competitive Sports: Evidence from} the English Premier League $\ldots \ldots \ldots \ldots \ldots \ldots \ldots \ldots \ldots \ldots \ldots \ldots \ldots$

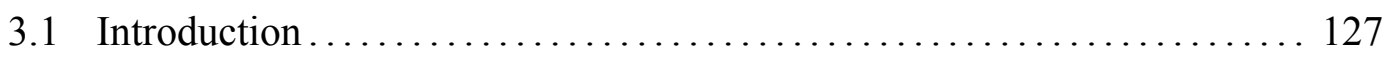

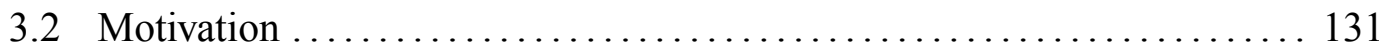

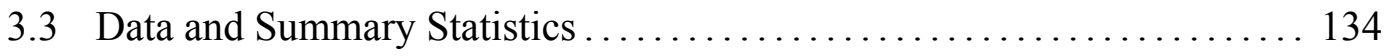

3.4 Methodology ...................................... 140

3.4.1 Probit Estimation .............................. 140 
3.4.2 Multinomial Logit Estimation ....................... 144

3.4.3 Survival Analysis ............................... 146

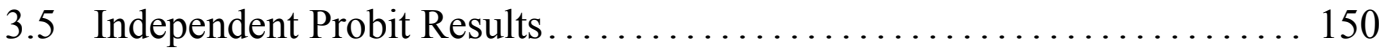

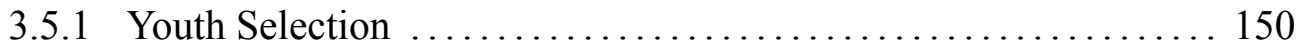

3.5.2 Foreign Selection ............................... 154

3.6 Multinomial Logit Results .............................. 157

3.7 Survival Analysis Results ................................ 159

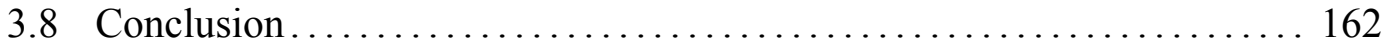

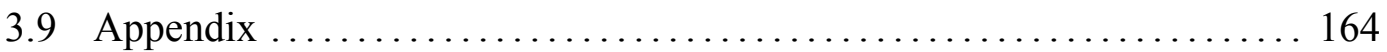

3.9.1 Heteroskedastic Probit Model ............................ 164

3.9.2 Note on the Hausman Test of IIA . .................... 165

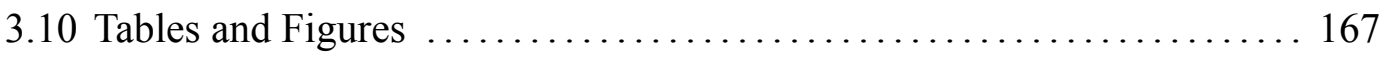

4 Concluding Remarks ................................ 174

References ............................................ 176 


\section{List of Tables}

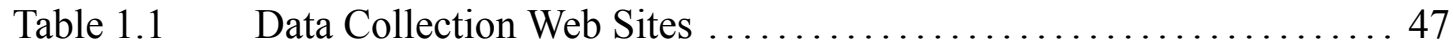

Table $1.2 \quad$ Duration Models Considered ........................... 48

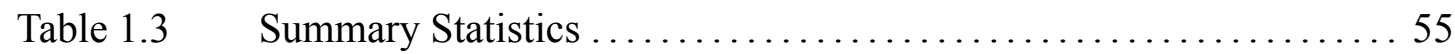

Table 1.4 Hazard Summary for Players' Career and HT Spell Duration ...... 55

Table $1.5 \quad$ AIC and BIC Scores for Career Duration $\ldots \ldots \ldots \ldots \ldots \ldots \ldots \ldots$

Table 1.6 Gompertz Model Career Duration Results .................. 56

Table $1.7 \quad$ Cox Model Career Duration Results ..................... 57

Table $1.8 \quad$ Cox Model HT Spell Duration Results $\ldots \ldots \ldots \ldots \ldots \ldots \ldots \ldots \ldots$

Table $1.9 \quad$ Split Sample Tests ................................. 59

Table $2.1 \quad$ Player Entries by Bosman and Source $\ldots \ldots \ldots \ldots \ldots \ldots \ldots \ldots$

Table $2.2 \quad$ Sample Summary Statistics $\ldots \ldots \ldots \ldots \ldots \ldots \ldots \ldots \ldots \ldots \ldots \ldots \ldots \ldots \ldots \ldots$

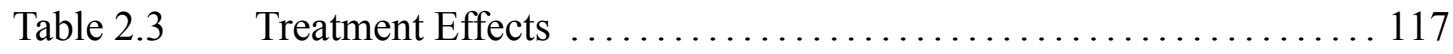

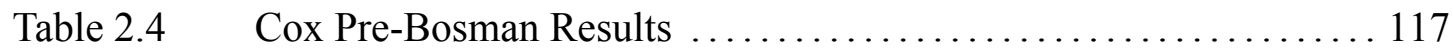

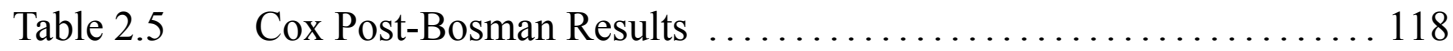

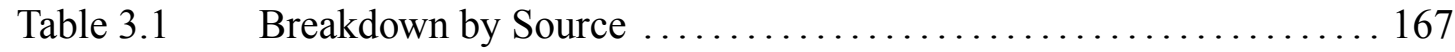

Table 3.2 Youth Breakdown by International Experience $\ldots \ldots \ldots \ldots \ldots \ldots 7$

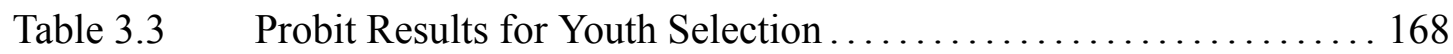

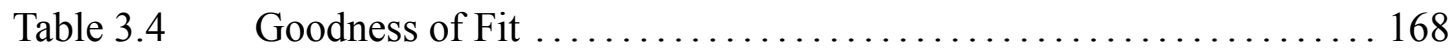

Table $3.5 \quad$ Link Test for Misspecificatio - Youth $\ldots \ldots \ldots \ldots \ldots \ldots \ldots$

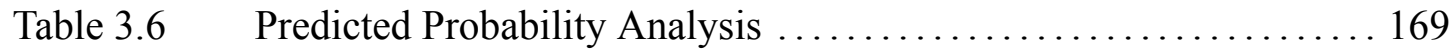


Table $3.7 \quad$ Probit Results for Foreign Selection $\ldots \ldots \ldots \ldots \ldots \ldots \ldots \ldots \ldots \ldots$

Table $3.8 \quad$ Multinomial Logit Results $\ldots \ldots \ldots \ldots \ldots \ldots \ldots \ldots \ldots \ldots \ldots \ldots$

Table 3.9 Cox Semiparametric Survival Results $\ldots \ldots \ldots \ldots \ldots \ldots \ldots \ldots 17$ 


\section{List of Figures}

Figure 1.1 (A) Career Duration Empirical Survivor Functiom, (B) Career Duration

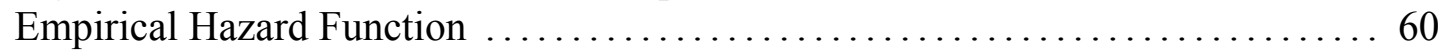

Figure 1.2 (A) HT Spell Duration Empirical Survivor Function, (B) HT Spell Duration Empirical Hazard Function $\ldots \ldots \ldots \ldots \ldots \ldots \ldots \ldots \ldots \ldots \ldots \ldots \ldots \ldots$

Figure 1.3 (A) Empirical Hazards by Youthteam for Career Duration, (B) Empirical Hazards by Youthteam for HT Spell Duration ................. 62

Figure 1.4 (A) Empirical Hazards by Position for Career Duration, (B) Empirical Hazards by Position for HT Spell Duration $\ldots \ldots \ldots \ldots \ldots \ldots \ldots \ldots \ldots \ldots \ldots \ldots$

Figure 1.5 (A) Gompertz Career Duration Baseline Hazards, (B) Gompertz Career

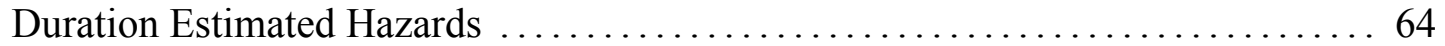

Figure 1.6 (A) Cox Semiparametric Baseline Hazards for Career Duration, (B) Cox Semiparametric Estimated Hazards for Career Duration ............... 65

Figure 1.7 (A) Cox Career Duration Goodness of Fit Test, (B) Cox vs. Gompertz

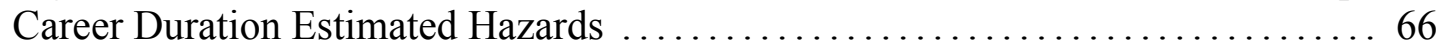

Figure $1.8 \quad$ (A) Cox Semiparametric Baseline Hazards for HT Spell Duration, (B) Cox Semiparametric Estimated Hazards for HT Spell Duration . . . . . . . . . . 67

Figure 1.9 Cox HT Spell Duration Goodness of Fit Test............... 68

Figure $2.1 \quad$ Graph of bin means for Apps $\ldots \ldots \ldots \ldots \ldots \ldots \ldots \ldots \ldots \ldots$

Figure $2.2 \quad$ Graph of bin means for MPG $\ldots \ldots \ldots \ldots \ldots \ldots \ldots \ldots \ldots \ldots$

Figure 2.3 (A) Mpg for 1 year bandwidth, (B) Mpg for 2 year bandwidth, (C) Mpg for 3 year bandwidth, (D) Mpg for 4 year bandwidth .............. 120

Figure $2.4 \quad$ (A) Apps for 1 year bandwidth, (B) Apps for 2 year bandwidth, (C) Apps for 3 year bandwidth, (D) Apps for 4 year bandwidth .............. 121

Figure 2.5 Empirical Hazards for Both Groups $\ldots \ldots \ldots \ldots \ldots \ldots \ldots \ldots \ldots$ 
Figure $2.6 \quad$ Pre-Bosman Baseline Hazards $\ldots \ldots \ldots \ldots \ldots \ldots \ldots \ldots \ldots \ldots \ldots$

Figure $2.7 \quad$ Pre-Bosman Estimated Hazards ...................... 123

Figure $2.8 \quad$ Pre-Bosman Goodness of Fit....................... 123

Figure 2.9 (A) Post-Bosman Baseline Hazards, (B) Post-Bosman Estimated

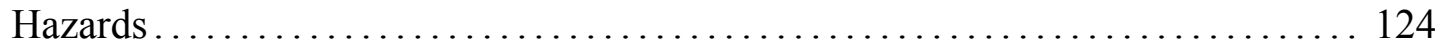

Figure 2.10 Post-Bosman Goodness of Fit Test $\ldots \ldots \ldots \ldots \ldots \ldots \ldots \ldots \ldots$

Figure 2.11 Estimated Hazards for Both Groups $\ldots \ldots \ldots \ldots \ldots \ldots \ldots \ldots \ldots$

Figure 2.12 Kolmogorov-Smirnov Test for Stochastic Dominance ......... 126

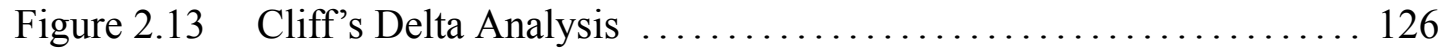

Figure $3.1 \quad$ (A) Empirical Survivor Functions by Youthteam, (B) Empirical Hazard

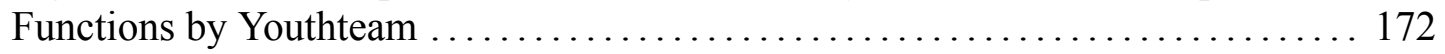

Figure 3.2 (A) Cox Baseline Hazards for Youth Players, (B) Cox Estimated Career Duration Hazards for Youth Players . . . . . . . . . . . . . . . . . . . . . . 173 


\section{Introduction}

The study of sports economics is a growing research area with a lot of poten-

tial. The sports industry sector is an increasing contributor to GDP in many countries worldwide, particularly in the EU with a share in the national economies which is comparable to agriculture, forestry and fisherie combined. Moreover, its share is expected to rise in the future. Overall the sports sector accounts for $2 \%$ of the EU global GDP, while the total employment generated by sports activities is 7.3 million-equivalent to $3.5 \%$ of the total EU employment (2014 European Commission report). Major sporting events (i.e. World Cup in soccer) significantl improve the local infrastructure of host countries and drive tourism revenues. Sports participation is also an important factor in the well being and overall health of people, which presents a non-measurable social benefi that cannot be overlooked. From an academic perspective, sports provide unique opportunities to test economic theories, especially in the fiel of Labour Economics.

This thesis is comprised of three separate chapters in the fiel of sports economics, with a particular focus on European soccer and the English Premier League. All three chapters are empirical works, with Chapters 2 and 3 extending the research question in line with relevant labour economics theory. Two uniquely collected data sets, among other things, serve as a major contribution to this thesis and literature in general. 
The paper presented in Chapter 1 is the firs of its kind in economic literature directly addressing the impact of different youth training (education) programs on the career success of professional athletes. The empirical analysis in this chapter relies on a proprietary data set of post-war (WWII) English soccer players trained at youth level by one of the clubs considered. Duration models in the spirit of Abbrig and Van den Berg (2003) are employed to estimate local treatment effects of different training programs on player survival (career and spell duration) in the top European leagues. The finding of this research study identify the effects of different education/training programs, among other observed and unobserved factors, in explaining the labor market differences of individual soccer players measured by the length of their professional career. Certain clubs, with a well established reputation in developing youth talent, outperform others in terms of producing and evaluating the ability of their youth players to succeed in top European leagues. The spell duration results suggest that some smaller clubs outperform larger clubs in terms of producing and holding on to homegrown talent, which would be a necessary condition for them to remain competitive in light of their lagging financia resources that limit their transfer market activity.

The paper presented in Chapter 2 is motivated by the positive-spillover hypothesis and certain contest theory literature, and analyses the impact of the Bosman ruling on the competitive nature of new entrants to the English Premier League. Another 
proprietary data set is collected in 4-year windows around the Bosman ruling (Dec., 1995) for new entrants to the English Premier league. Regression Discontinuity Design displays evident discontinuity in proxies for player productivity around the ruling; the results indicate there is a sharp positive jump in productivity measures for players entering after the ruling. Furthermore, an additional survival analysis is performed for the two groups of players that entered the market on either side of the ruling; the results suggest that post-Bosman entrants have a higher probability of survival or earning a first-tea spot at a top-division club. In line with the relevant labour economics theory, moving to a more homogenous competitive environment increases total quality/productivity of new entrants, via increases in talent level and/or effort exerted by competing players, leading to higher probability of survival in top European leagues.

Chapter 3 of the thesis utilizes the data sets from the firs two chapters in a different research context. This chapter is also the first to my knowledge, in economics literature that analyzes the labour theory of internal promotion vs. external recruitment in a professional sports environment. The data set and results from the firs chapter are used to create a unique categorical index that ranks different youth programs in the English Premier League in one of four tiers, and this index serves to identify the degree of youth orientation or "bottom-heaviness" for each of these clubs. Following this step, the data set from the second chapter is augmented and utilized to examine the hiring patterns of clubs when bringing in new and unproven Premier League 
players into their first-team Binomial and multinomial choice models are employed to analyze the probability of selecting players from the youth stream (internal hiring) into the first team for all clubs in the league in 4-year windows around the Bosman ruling; this allowed the analysis period to capture the most important recent institutional change in European sports. Robust results are established that support the notion that more reputable youth programs (bottom-heavy) provide more first-tea opportunities through internal sources, which is in line with the motivating theory and results from other industries. A separate survival analysis validates the results from the firs chapter in terms of youth training reputation of certain clubs, and establishes a presence of heterogeneity at youth club level that signals differences in player career prospects generated by their youth training and lends further support to the results of the paper presented in Chapter 1.

The tables and figures as well as the relevant appendices, are listed at the end of each chapter. Chapter 4 provides the concluding remarks for the thesis, while the overall bibliography for all three chapters is presented at the end of the thesis. 


\section{Chapter 1 \\ Youth Training Programs and their Impact on Career and Spell Duration of Professional Soccer Players}

\subsection{Introduction}

Linking education or specialized training with career success has been the subject of many economic studies in the past. Most articles focused on conventional types of education and schooling in assessing its return on investment. The sports industry has been growing rapidly worldwide, but the impact of education or training on professional success of players has been largely unexplored in economic literature. Youth System is a sports term that refers to a youth investment program within a particular team/league that develops and nurtures young talent. Most sports in North America rely on the high school and collegiate system to develop young athletes, with the exception of hockey in Canada and baseball in the USA that offer many junior and minor leagues for youth development. On the other hand, most team sports in Europe concentrate and invest in youth systems and almost every country has youth level leagues in multiple disciplines for young athletes to compete and further develop. 


\subsubsection{Motivation}

Youth academy soccer is an essential component in the development cycle of any aspiring young player. Those individuals selected at youth level are considered to have the necessary potential to become professional soccer players. Most of these youth players are trained and nurtured at academies of licensed soccer clubs, with the hope of signing a professional contract with the club when they turn 17 . This is the grassroots level of soccer that teaches young players all of the basics of becoming a soccer player. The basics provided at youth level are intended to equip the players with the necessary tools that help them establish foundations upon which to build on for the rest of their careers. Clubs throughout Europe have invested a lot of time and money ${ }^{1}$ in their youth academies, especially in recent decades, as the benefit of running a successful youth academy have been realized. Clubs have numerous scouts and youth coaches that assess potential players at each step of their development process. Investing in youth academy training is one of the factors that enters a club's objective function. $^{2}$

The Bosman ruling (December 15, 1995) was a major institutional change in European sports, effecting professional teams and youth academies as well, in a sense that EU players were now treated as domestic in other EU countries. The ruling

\footnotetext{
1 One recent example is the Chelsea Football Club (England) who has invested millions of pounds into a new academy center.

2 A club's objective function could be driven by win-maximization and/or profit-maximizatio forces. A successful youth training program will be a part of an objective function of any club, regardless of its weighting on winning and profit
} 
signaled a shift in the competitive structure and modernization of the game as we know it today.

Football academies were set up in England in 1998, prior to which most clubs had "centers for excellence" for talented players ${ }^{3}$. In the modern academies, boys are signed anywhere from ages 8 to 16 . These boys are offered yearly "retain" or "release" contracts until the age of 12 , at which time these contracts are extended to two years. At 16, boys become full-time scholars and they become paid apprentices. The English Football (FA) has rules that 8-11 year olds have to live 60 minutes traveling time away from the training ground, 12-16 year olds 90 minutes away, ${ }^{4}$ and at 16 the 90 minute rule is abolished and players can be signed from anywhere in the EU. ${ }^{5}$ Training intensity also increases with age; minimum of 3 hours per week for 8-11 year olds, minimum 5 hours for 12-16 year olds, after which the intensity increases significantl to almost professional standards. According to the 2012 European Club Association (ECA) report on youth academies in Europe, the majority of clubs surveyed have around 220 players in the youth academy and work with 3-4 different

3 Youth training and competition among clubs for top talent has always existed, but it evolved over time parallel to changes at the professional level. The changes in 1998 in England really focused on increasing the competitiveness of youth leagues and moving away from regional leagues.

4 These rules expose clubs to uneven pools of talent available to them. For example, some of the London clubs have 15-20 million people living 90 minutes away, while a club like Southampton is much more restricted considering half of its radius is in the English Channel. Yet, Southampton is one of the most well-know youth academies in the world, which indicates that the training quality in the academies is a key factor that cannot be overlooked.

5 Prior to the Bosman ruling, once the 90 minute rule was abolished at age 16, players could be sourced from anywhere in the UK. Therefore, the Bosman ruling expanded the talent pool available to clubs at youth level, and increased investment in terms of size and scope of their scouting network. This certainly benefite the wealthier clubs to a greater extent, while smaller clubs could not devote similar resources in scouting foreign talent. 
age groups, $75 \%$ of the players come from the region less than one hour away, $60 \%$ of the academies have foreign players but only $3 \%$ of the players in the oldest age group come from another country. In addition, $50 \%$ of the clubs surveyed spend less than $6 \%$ of their budget on their youth academy, there are generally 6 academy players that are playing on the first-team and on average $8.6 \%$ of the youth players (from the oldest age group) signed their firs professional contract with the club that trained them.

Developing home-grown talent could be a viable long-term strategy for certain clubs that want to be successful, financiall sound, and competitive in the long-run. Each soccer club faces an economic dilemma in search of talented players, dynamically optimizing its investments between youth academies and the transfer market subject to a budget constraint. Producing high-performing players through the youth system can ease the financia strains of a soccer club by avoiding large spending in the transfer market and unknowns associated with out-of-club players. Barcelona Football Club (Spain) is a perfect example of success brought on via the success of their youth academy (La Masia). They have won all major honors in domestic and international soccer competitions in recent years, and the majority of their firs team players are products of their youth academy. The success of their youth program has put less strain on their transfer market expenditures and allowed for more fl xibility in their financia structure. ${ }^{6}$ Not only does a successful youth academy ensure a con-

6 They were one of the only teams in the world without an officia sponsor on their shirts until 2010, showing the financia strength of the club. 
tinuous $\mathrm{fl} \mathrm{w}$ of home grown players into the firs team, it can also serve as a profi driver when selling a player's rights in the transfer market. In addition to the finan cial benefit of running a successful youth program, there are intangible benefit in terms of bonds and loyalty created between the youth player and his home club (particularly with the fans) that could last for the player's entire career. This could be extremely beneficia to all soccer clubs, in particular to smaller clubs because it allows them to stay competitive with larger and financiall superior clubs, at least for a certain period of time. From a youth player's perspective, knowing that his home team provides first-tea chances to its trainees adds incentive to increase his effort because their dreams of making it professional appear more realistic and attainable at these clubs, inducing them to try harder and impress their coaches.

\subsubsection{Literature Review}

This chapter, to the best of my knowledge, is the firs of its kind in economic literature directly addressing the impact of youth training (education) programs on the career success of professional athletes. The spirit of the paper is based on Abbrig and Van den Berg (2003) who link the treatment effects literature to duration analysis and use as examples effects of training programs on unemployment durations. In this chapter however, while looking at the treatment effects on the treated we investigate also the heterogeneous effects associated within the treated group. 
Frick, Pietzner, and Prinz (2007) attempt to answer several questions simultaneously, mainly focusing on the effect of changes in the German soccer institutional environment on individual player career duration. They estimate a Cox Proportional Hazard model based on data collected for all players appearing in the German First Division (Bundesliga) during a 40 year period. Their finding indicate that institutional changes have a strong effect on individual players' careers, career duration of goalkeepers is longer than for the other positions considered, and that players from certain regions (i.e., Eastern Europe) might suffer from discrimination. Goddard and Wilson (2009) estimate a competing risk model and fin evidence of racial discrimination in English football. Ohkusa (2001) examined the quit behavior of Japanese baseball players, while Atkinson and Tschirhart (1986) studied the determinants of career length for NFL players. Others, like Groothuis and Hill (2004) have analyzed the exit discrimination of black players in the NBA.

\subsubsection{Short Summary of the Chapter and its Contribution}

The empirical analysis in this chapter relies on a uniquely collected data set of postwar (WWII) English soccer players trained at youth level and appearing at least once for one of the clubs considered. ${ }^{7}$ The aim of this research study is to estimate the effects of different education/training programs, among other player-specifi observed

7 The data does not identify a lower proportion of youth players appearing for larger clubs (in fact, Manchester United youth products are the largest sub-sample in the data) considered in this study. Therefore, the analysis in this paper is comparing median players, from the ones that make it pro, from each academy to the extent possible. 
and unobserved factors, in explaining the labor market differences of individual soccer players measured by the length of their professional career. In the absence of more appropriate measures, the economic returns to players are estimated by players' career and spell durations. Assuming that players earn income ${ }^{8}$ in each year of their professional career, their income generated will be greater the longer their career duration is. ${ }^{9}$ In addition to general differences in the quality of youth programs, it is important for clubs to determine at an early stage which players have the physical and mental attributes to succeed at the top level. This analysis isolates the home-grown players that a club perceives as future high performers and evaluates differences between clubs based on the ability to assess their youth product in terms of career potential at the top level of European soccer. Essentially, we are measuring the differences in the internal mechanism of the 16 clubs in assessing their home-grown talent at the fina stage of youth development (when a player turns 18 and a decision has to be made whether to sign or release him). From an economics perspective, clubs in this study will sign home-grown players if they assess they have the necessary talent and physical attributes to play professionally in the future. Thus,

8 In the modern era, especially since the Bosman ruling, players have multiple sources of revenue besides their wage that may affect their professional careers and duration. For example, players now earn income from selling/licensing their image rights, sponsorship contracts, etc. However, most players, other than superstars, still rely on their wages as the major source of revenues.

9 While there are differences in wages earned by players, these differences are reduced by including only top level players from teams that were the most frequent English top-tier participants during the study period. Goddard and Wilson (2009) and Dobson and Goddard (2001), among others, provide evidence that player wages are highly correlated with divisional status. This sample homogeneity will reduce the impact of differential rents and team stature on the duration of soccer players and will aid the identificatio of the quality of a given youth training program. 
they can realize a return on their investment of training a player in two ways: either playing him in their first-team or selling his rights in the transfer market.

Fans in soccer are generally fonder of home-grown players in their local teams, and their inclusion in the firs team can have a positive impact on attendance generating economic rents for clubs and players. The specifi data on player spell durations allows for assessing the sub-group differences in a player's tenure at his parent club. Generating quality players through youth training is important, but it is just as important to establish which clubs have the ability and willingness to hold on to their youth products the longest, at least if win maximization enters a club's objective function; This analysis can ascertain whether certain clubs are more successful than others in creating loyalty or bonds with the players they brought up through their youth system, conditional on the player's ability to play at the top level for more than one club. ${ }^{10}$ In other words, the spell duration analysis can shed some light on the return of investment achieved by the clubs through their youth academies. The implications of this analysis might be different for larger versus smaller clubs. The competitive first-tea environment at larger clubs makes it much more difficul for youth players to establish themselves and have long spells with their parent club. Therefore, the results for larger clubs cannot be interpreted in the same fashion as the results for smaller clubs in the study because larger clubs have more leverage in the trans-

10 This assumption that players have a choice of top-level club is reasonable because quality players will survive in top leagues or be picked up by other top-level teams regardless of the academy they originate from, and lower quality players will resort to playing the majority of their career at lower level leagues. 
fer market to acquire quality players. One would expect that the less reputable clubs in this study would be more reliant on home-grown players because of their inability to compete with larger clubs in the transfer market. On the other hand, smaller clubs might not be able to keep their most talented youths because they can't compete fi nancially with top clubs with superior resources available for player salaries. On an average player basis one would expect that youth training provides an option for smaller clubs in this study through holding on to their talented home-grown players.

Using individual specifi characteristics and productivity measure proxies, such as season appearances, allows for the identificatio of conditional duration patterns for an individual player. Consequently, reduced form duration models are estimated to model a player's career duration, as well as his spell duration with the club who trained him at youth level. A benchmark model, that includes individual specifi characteristics, is estimated first Subsequently, productivity variables are included in the model, while the complete model will account for nonlinear functional form of relevant continuous covariates and unobserved heterogeneity, if applicable.

The rest of the chapter is organized as follows: Section 2 discusses the data set and provides some summary statistics; Section 3 discusses the methodology employed; Sections 4 and 5 discusses the results of the empirical analysis for career and home team spell duration; Section 6 discusses endogeneity issues and Section 7 concludes the chapter. 


\subsection{Data Set and Summary Statistics}

Despite the abundance of soccer data available today, information pertaining to players at youth level is not readily available, at least not publicly. Therefore, testing for general effects of attending different youth academies is restricted by data availability.

\subsubsection{Data Structure}

The data collected for this chapter allows for the testing of the local heterogeneous effects of attending different youth academies on a player's career duration in top European leagues. ${ }^{11}$ Specialized academies recruit youth players anywhere from 8 to 18 years of age. ${ }^{12}$ The data set in this study consists of those players that were trained at youth level, signed professionally at 18, and made an appearance for one of the clubs considered. ${ }^{13}$ The selection is assembled using youth trainees from 16 English clubs ${ }^{14}$ in the post-War period commencing in 1946 and ending with the

11 The top leagues considered for this study were from the following countries: England, Spain, Italy, Germany, and France.

12 During the period of investigation there were restrictions in place that didn't allow youth players to attend an academy if they lived more than an hour away from it. However, there were exceptional cases where some teams got around this and would move entire families for the more talented players. As these incidents where rather few than many, clubs were somewhat restrained to training local talent and this reduces the bias of larger clubs attracting the best talent from around the country and smaller teams having to rely on mediocre talent pools.

13 The fact that data are not available for youth trainees that didn't sign professionally for any club or made an appearance for one of the 16 teams does not allow for testing the overall success (general effects) of competing youth academies.

14 The 16 clubs included in the study are: Arsenal, Aston Villa, Chelsea, Coventry City, Everton, Leeds United, Liverpool, Manchester United, Manchester City, Newcastle, Nottingham Forrest, Sheffiel Wednesday, Southampton, Tottenham, West Bromwich, West Ham. 
2010/2011 season. These clubs were selected based on data availability and their extended presence in the top tier of English soccer over the period of study. The data, as presented, will be less likely to induce selection bias in the results, as the selected clubs are among the most frequent participants in the top tier of English soccer during the period of interest. ${ }^{15}$ The selection of the most "successful" (in terms of top-tier participation) clubs reduces the bias associated with successful clubs being able to influenc youth level participation in their local jurisdictions. In addition, a player's relative abundance of human capital ${ }^{16}$ and natural talent should assure a presence in the top leagues, regardless of whether his home team, or any other team that he plays for, gets relegated from the top league. The clubs examined in this chapter were all facing the same rules and regulations and youth selection criteria. Generally speaking, there is no evidence that the athletic attributes of the pre-selected youths were different throughout the country, and international (particularly non-EU) players do not represent a significan portion of the sample to distort the results. This assumption is more grounded considering the players included are youth trainees that sign professional contracts at the age of 18 , while appearing for the firs team in the process, and represent the cream of the crop from each academy examined. ${ }^{17}$

15 The top tier of English soccer was called Division 1 until the Premier League was established in the $1992 / 1993$ season.

16 In soccer, human capital can be ascertained by a level of tactical knowledge and understanding that allows players to embrace tactical changes imposed by new managers or new systems of play, in addition to natural talent.

17 Less than 1 percent of all youth trainees actually end up making a living from playing soccer, and this percentage is even lower for top-league participants. The data contain only a specifi subsample of these academies, which allows us to estimate a local effect only. It is reasonable to assume that the sample selection introduces an upward bias in terms of duration. 
As such, the sample itself reduces the effect of unobservables due to differences in player natural talent and athletic capabilities.

The data collected include detailed player level information and statistics that can be structured into two groups:

1. Variables with initial conditions and personal characteristics: player's name, youth/training program attended (Youthteam), age at firs appearance in a top league (ageentry), year in which the player firs appeared in a top league (yearentry), nationality of the player, and position played for the majority of his career. ${ }^{18}$

2. Productivity measure proxies: number of top league seasons played at each club in his career, total number of seasons played in top leagues, per season average firs team top league appearances in a player's career (avgapps), total career appearances in the top league, average goals scored per season (avggoals), international appearances for national team (intcaps), and international goals scored.

\subsubsection{Data Generation}

The data was gathered though a variety of Internet sources, the main source being

18 Six positions were considered for the purposes of this paper: Forwards, Midfielders Wingers, Central Defenders, Full Backs, and Goalies. 
http://www.neilbrown.newcastlefans.com/, a site providing A-Z player data for post-war English clubs. Every player that made at least one appearance for an English club during this period is accounted for, some with more detailed information than others. The players' statistics are drawn from Berry Hugman's series of books (19842005), “The Premier and Football League Player's Records". The source data were of particular importance because they distinguish if a player was trained at youth level ${ }^{19}$ by that particular club. This information was used as a proxy for receiving some $^{20}$ education/training prior to starting a professional career and define the initial conditions of the player-level data, allowing for accurate tracking of the careers of 1121 professional players during the study period.

Most of the clubs analyzed did not have a constant presence in the top English league and experienced some movement within the English league system through relegation and promotion. ${ }^{21}$ Numerous other Internet sources, outlined in the Appendix, were engaged to gather the relevant statistics from the top European leagues for each player in the data set. Most players dropped from the risk pool were due to the inability of segmenting their top level club-specifi spell data in the event the spell

19 Youth trainees, junior players and apprentices are all treated as players that received training by their parent clubs prior to signing a professional contract. These terms were used interchangeably prior to 1998 , when youth academy structure commenced. The data contains information on participation at the last stage of youth development, as an apprentice signed at 16 .

20 We do not have information of the length and intensity of youth training, which could potentially be an important factor for a player's career duration, and particularly the bond/association with the club that trained him.

21 The bottom two or three teams of every league, except the bottom league, got relegated to the lower league and the top 2 or 3 teams of every league, except the top league, got promoted to the higher league on a yearly basis. A similar system is in place for other Western European countries. 
carried over to lower level leagues in any particular country in which the player appeared. The data track a player's spell duration for each club that he played for in the top leagues during his entire career, regardless of exit and re-entry as only the relevant seasons and statistics in the top tier are accounted for. Any fina departure 22 from the leagues examined was considered as an exit from the top tier competitive soccer market. ${ }^{23}$

\subsubsection{Broader Applicability}

The unique structure of the data also permits multiple other questions to be broadly examined, including:

- The impact of a particular choice of position on the duration of a player's career. Players and coaches make choices early on in a player's career on the position to be assumed by that player, and that choice might impact the player's career duration because each position is demanding in its own way. More technically gifted players are typically assigned midfiel and forward roles on any given team. Certain positions, like full-backs and wingers are reliant on speed and stamina, so one would not expect players

\footnotetext{
22 The players examined exit the top league due to a permanent transfer to the lower level leagues or retirement. A player's health is a key factor in his career length, and the data accounts for it by excluding all players that exit or are forced to retire due to injury, although this was not a significan portion of the data to begin with.

23 The aim of this study is to assess the differences of youth programs attended on a player's career and spell duration at the top professional level. This is why the data doesn't track players if they drop out of the top league, even though we are aware that they could potentially still be playing professionally in the lower leagues and earning income.
} 
at these positions to play past a certain age when those required attributes start declining. In addition, certain positions in soccer are subject to more scrutiny than others because their performance is tied to easily identifiabl statistics, like the number of appearances, goals scored, etc. For example, the productivity of forwards is generally measured by the number of goals they score. The same measure of productivity cannot be applied to the other positions in soccer. In addition, a player's adaptability to other positions can extend a player's career by assuming other roles required by management and ensuring a firs team presence, ${ }^{24}$ but this is outside of the scope of this study.

- The competitive structure of English soccer has changed over the period of study. For example: (i) In 1973, the Football league announced that three teams (instead of two) would be relegated from the top two divisions; (ii) In 1981, a change was initiated so that three points were awarded for a win instead of two; (iii) In 1992, the Football Association created the FA Premier League replacing the Football League First Division allowing for 22 clubs to compete instead of $20{ }^{25}$ (iv) In 1996 , the Bosman ruling impacted all European competitions by increasing the number of foreign players allowed per team and allowing for free movement without any restrictions on clubs for players with passports from EU countries. These institutional changes could

24 This study considers the position a player assumed for most of his career, which does not include a measure for the adaptability factor of players.

25 Later reduced back down to 20 teams in the Premier League. 
have opposing effects on a player's survival in the top league; one can assume that increasing the number of teams in the top division will provide a player with a higher chance of retaining a spot in the top league, while increasing the number of relegated teams can have a negative effect on a player's chances of staying in the top competition. We hope to indirectly extrapolate the effect of some of these changes in the competitive structure of English soccer by testing the effect of the year of entry on a player's career duration.

\subsubsection{Summary Statistics}

Basic summary statistics for the continuous variables are presented in Table $1.3 .^{26}$ There are 13 players who were trained by a club but did not make a first-tea appearance for that club in the top leagues. ${ }^{27}$ The mean HT (HT refers to home team that trained a particular player at youth level) spell duration is not much lower than mean career duration, which suggests a certain link or loyalty exists between players and the clubs that provide youth training for them. Maximum career duration is 21 years and maximum HT spell duration is 19 years. The youngest player to make a first-tea appearance for a club in this study was 15 , while the oldest was 29 . Table 1.4 outlines the detailed information for all player exits at each observed time period for career duration and home team spell duration. Exits are a little more frequent in

26 We consider all productivity measures as being continuous variables as they have ranges that exceed 100 .

27 This could be because the club was in the lower league at the time a player was there, or that the player was signed professionally by another club examined straight out of his youth program. 
the early years for the spell duration versus career duration. Graphs of the empirical survivor and hazard functions for career and HT spell duration are presented in Figures 1.1 and 1.2, respectively. The empirical hazard graphs show a strong positive duration dependence for the players career and HT spell duration, indicated by the upward sloping curves, which is consistent with downward sloping survivor functions. This is not surprising considering the age and physical limitations of individual players, as well as intense competition in the marketplace. The main observable difference between the career and HT spell duration empirical hazards is in the hazard levels of the two curves; The HT spell duration hazards are higher than career duration hazards in general. In addition, the career duration empirical hazard displays some variability in the upward sloping latter stages of the analysis (between duration times 17 and 20), whereas the empirical hazard for HT spell duration slopes upward sharply without notable variability after 15 years.

Visual and formal Wilcoxon and Log-rank tests $^{28}$ indicate that subgroup differences are present for youth team attended and position played. Figure 1.3 (A,B) illustrates the sub-group differences at club level for career and HT spell duration, respectively. ${ }^{29}$ Figure $1.4(\mathrm{~A}, \mathrm{~B})$ presents additional visual analysis that shows there are subgroup differences at the position level for career and spell duration; estimated hazard rate for goaltenders is lower than for the other positions for the majority of

\footnotetext{
28 These tests are non-parametric statistical hypothesis tests used when comparing two or more related samples, matched samples, or repeated measurements on a single sample to assess whether their population mean ranks differ.

29 The figure display 5 out of the 16 clubs that are part of this study, with no particular reason for the choice of clubs displayed. The graph with all 16 clubs is too condensed for interpretation.
} 
the duration time analyzed, and that forwards have the highest hazard rate among the positions considered. Similar type of analysis resulted in the selection of Sheffiel Wednesday as the appropriate youth team benchmark in career and HT spell duration analysis, since its estimated hazard rates appeared to be stable and on the bottom end of all of the clubs considered. These results indicate that the different youth teams have a unique effect on the hazard rate, and that the objective of this study can be explained by the data.

\subsection{Methodology}

Our analysis is based on duration models that are used to estimate the hazard rate (the instantaneous probability of exit a given state), ${ }^{30}$ which is define as:

$$
h(t)=\lim _{\triangle t \rightarrow 0} \frac{\operatorname{Pr}(t \leq T<t+\Delta t \mid T \geq t)}{\triangle t} .
$$

A general specificatio for a reduced form hazard model when we condition on observable individual characteristics $X$ is represented as:

$$
h(t)=h_{0}(t) \phi(X \beta)
$$

where $h_{0}(t)$ is called the baseline hazard and is a function of $t$ only with all covariates set to zero and $\phi(X \beta)$ can be interpreted as a scaling factor, typically specifie in an exponential form. In our case the duration is measured in years. Given that we are estimating careers duration (which may exceed 20 years), we consider esti-

\footnotetext{
30 Time is measured by seasons/years played, and exit rate is determined by any permanent departure
} from the top-level leagues considered in this paper. 
mating the interval to an exit, which requires a continuous treatment of time. ${ }^{31}$ To account for potential model specificatio errors, the reduced form hazard specifica tions in this analysis are modeled both parametrically and semiparametrically (see the Appendix for details about the methods used), while conditioning on individual specifi covariates, including dummies for the player's youth team, position played, and nationality. Nonparametric regression techniques for testing nonlinear functional forms of continuous covariates are applied to improve model specification The effect of unobservables is reduced by accounting for individual-specifi unobserved heterogeneity, where applicable.

\subsubsection{Functional Form of Covariates}

Standard tests for nonproportionality of hazards are quite powerful, but they can also detect a variety of other specificatio errors, including the misspecificatio of the functional form for the covariates. This is particularly important for the Cox semiparametric model, but it shouldn't be overlooked for the parametric models as well. Testing for nonlinear functional forms of continuous covariates is a possible solution to improve model specification This test is usually done by including fractional polynomials of the covariate, or by nonparametric methods such as splines. Keele (2010) notes that very few articles in the literature test for nonlinear functional form of continuous variables (linear form is taken as given), with no results of nonlinear-

31 We did consider discrete time models, but they did not give better predictions. 
ity tests using the nonparametric spline technique. Keele $(2010)^{32}$ notes that the close relationship between functional form and the PH test lends extra urgency to modeling of any nonlinearity, because mis-specificatio of the functional form can lead to inappropriately rejecting the $\mathrm{PH}$ assumption.

Productivity measures might have different implications in the career duration model and in the HT spell duration model. While we still expect a positive correlation between productivity and duration, HT spell duration can have opposite correlation with productivity depending on the team a player originates from. If a player originates from a smaller and historically less successful team in our sample, increased productivity might result in shorter spell duration (negative correlation) with his home team as he will attract the attention of larger and more successful clubs that try to engage his rights in the transfer market. For the larger and more successful clubs in our sample, we would expect a strong positive correlation between productivity and spell duration. This reasoning suggests that there might be a critical point (inflection for productivity of players in small teams, where the correlation with spell duration changes from positive to negative as the player outgrows his current team. On the other hand, productivity can also be a factor differentiating career duration of players originating from larger and smaller clubs. A certain level of productivity in a smaller club might spell an end of a player's duration in the top league

32 Keele's paper uses simulations and well-known empirical examples from the literature to demonstrate the importance of testing for nonlinear functional forms. He demonstrates that, if this issue is ignored, the analyst might be mis-led by incorrectly relying on results from the nonproportionality test. 
and make him more suitable for lower league competitions, while the same level of productivity at a larger club might result in a player's movement to smaller clubs in the top league that extends a player's career duration. Considering the above, it is not surprising that the most suitable career and HT spell duration models incorporate nonlinear effects for proxy productivity covariates (i.e. appearances per season).

We account for nonlinearity in the productivity measure using restricted cubic spline functions (Royston and Sauerbrei 2007) define as follows:

$$
\begin{aligned}
s(x) & =\beta_{00}+\beta_{10} x+\sum_{j=1}^{m} \beta_{j}\left\{\left(x-k_{j}\right)_{+}^{3}-\lambda_{j}\left(x-k_{\min }\right)_{+}^{3}-\left(1-\lambda_{j}\right)\left(x-k_{\max }\right)_{+}^{3}\right\} \\
& =\phi_{0}+\phi_{1} x+\phi_{2} v_{1}(x)+\ldots+\phi_{m+1} v_{m}(x)
\end{aligned}
$$

where $k_{\min }$ and $k_{\max }$ represent two boundary $\operatorname{knots},{ }^{33} \phi_{0}=\beta_{00}, \phi_{1}=\beta_{10}$ and $\phi_{j+1}=\beta_{j}, v_{j}(x)=\left(x-k_{j}\right)_{+}^{3}-\lambda_{j}\left(x-k_{\min }\right)_{+}^{3}-\left(1-\lambda_{j}\right)\left(x-k_{\max }\right)_{+}^{3}$ for $j=1, \ldots, m$, and the plus function $(x-k)_{+}$is define as $x-k$ if $x \geq k$ and 0 otherwise.

In particular for each continuous covariate, the most complex permitted regression spline model is chosen (determined by the degrees of freedom assigned to the function) that has $m+1$ degrees of freedom (df), where $m$ is the maximum number of knots to be considered and $m=0$ represents a linear function. The most complex model, $M m$, is compared to the null model (omitting $x$ ) using a chi squared test with $m+1 \mathrm{df}$. If the test is not significan at $(\alpha)$ level, the procedure stops and the regressor $x$ is eliminated. Otherwise, the fi of $M m$ is compared to $M 0$, and if the difference is not signif cant at the $(\alpha)$ level, $M 0$ is chosen by default and the algorithm

33 The cubic regression spline is restricted to be linear beyond these boundary knots. 
stops. The procedure continuous in this fashion until either a test is not significan or all the tests are significant in which case $M m$ is the fina choice.

\subsubsection{State Dependence versus Unobserved Heterogeneity}

There are two possible sources for observing duration dependence: state dependence or true state dependence (TSD) and unobserved heterogeneity or spurious state dependence (SSD). The notion of SSD amounts to observations being conditionally different (heterogeneous) in terms of their hazard because individual specifi unobserved characteristics. Models that don't incorporate unobserved heterogeneity assume that all observations with the same values for all covariates are identical. The presence of SSD in models that don't account for it will result in model misspecification Unobserved heterogeneity can take two forms: at the individual observation level and shared by a group of observations with similar characteristics. This chapter models individual-level unobserved heterogeneity multiplicatively (in parametric models) relying on random effects models. ${ }^{34}$ Conditional on choosing the proper covariates and SSD distribution, the random effects models (frailty models) generate estimates positively correlated to the empirical hazard and correctly predicting its slope. This type of modelling is particularly useful in this chapter, given the possible biases associated with the sample of players gathered from these youth academies. The preferred model, when controlling for heterogeneity, should be the one

34 Appendix B outlines the frailty models considered and the assumed distributions for unobserved heterogeneity (Gamma vs. Inverse Gaussian). The terms "unobserved heterogeneity" and "frailty" are used interchangeably in the paper. 
with least variation in the unobservables. Gutierrez (2002) suggests that the interpretation of results is sensitive to the frailty distribution selected. Under TSD, prior experience plays a role in determining the probability of remaining in a given state. Positive TSD implies that the longer an individual is in a given state, the more likely he is to stay there, which is equivalent to negative duration dependence as the hazard rate falls or remains fla over time. Negative TSD implies that the probability of exiting a given state increases with time spent in that state, which is equivalent to positive duration dependence. Applying these notions to career length of individual soccer players would suggest that individuals with longer survival times increase their exit probability due to physical limitations imposed by age (negative TSD), but professional experience gained at a young age can result in positive TSD until a player reaches a certain age. The empirical hazard for career duration is relatively fla until season 11 of a player's career, after which it starts sloping upwards sharply. This suggests that prior experience is important (positive TSD) until year 11, and that the age factor dominates (negative TSD) after that time.

\subsection{Career Duration Results}

This section discusses the results from the analysis of individual player career duration and the determinants of their survival in the top leagues of Western European countries. All model specification include player-specifi variables as regressors that are divided into two groups: (i) player's initial conditions including personal 
characteristics, youth team that trained him, position played, and nationality, and (ii) player's productivity measures. ${ }^{35}$ The main objective in all specification is to assess the local effects of youth training on the duration of a player's professional career in the top leagues. Since all of the models presented in the study are proportional hazard (PH) models, the results are displayed as hazard ratios instead of the actual covariate coefficients Hazard ratios are very useful for interpretation purposes.

\subsubsection{Parametric Model}

The Generalized Gamma model was estimated to test whether one of the nested models is appropriate, and the results indicate that none of the nested parametric models are satisfactory. Notwithstanding these results, AIC and Baynesian Information Criteria (BIC) were used to adjudicate between nested and non-nested parametric models. The Gompertz parametric specification which is not nested in the Generalized Gamma model, had the most desirable scores. The AIC and BIC scores for all parametric models considered are presented in table $1.5 .^{36}$

Altogether there are f ve different Gompertz specification considered for career duration, M1: initial conditions only; M2: initial conditions and productivity measure variables, M3: initial conditions and productivity measures, with the addition of nonlinear functional forms, M4: initial conditions and productivity measures

\footnotetext{
35 Considering there are no direct measures of productivity, using data on appearances can serve as an exogenous proxy chosen by a club's manager based on his assessment of a player's productivity/contribution to the team.

36 AIC and BIC scores are provided for M2 and M3 specification only. The other specification considered render the same results.
} 
with heterogeneity and M5: full model including initial conditions, productivity measures, nonlinear functional forms, and heterogeneity.

\section{Main Results}

The results of these Gompertz specification are presented in Table 1.6. The specificatio with initial conditions (M1) serves as the benchmark model in this analysis. The youth team variables of primary interest become more significan as the model specificatio improves in terms of log-likelihood. For example, the M3 specificatio results in all youth team variable coefficient being significan at the given levels (with all but one significan at the 0.05 level). This findin indicates that the differences arising as a result of emerging professionally from one of the youth clubs examined serves as an important determinant in the career duration of professional soccer players. Taking one of the most renowned English clubs in history, Manchester United (Man U), the results suggest that attending and emerging professionally from their youth program would decrease the exit probability of players by anywhere from $31 \%$ to $43 \%$, depending on the specification compared to the players attending the Sheffiel Wednesday youth program (benchmark youth team in this study). Perhaps surprisingly, some of the historical powerhouses of English soccer (Man U, Chelsea, Everton, and Liverpool) lag behind some of the clubs (i.e. Leeds U and West Ham) with a lesser historical reputation in the top tier of English soccer. One would expect that players arising from one of the more reputable teams, and appearing for the firs team in the process, should be able to fin employment at the top 
level (in terms of career length) with more success than their counterparts emerging from less reputable teams. The results indicate that this is not the case and there is not a favorable difference in terms of quality of median players emerging from academies of larger and historically more successful clubs. This suggests that significan differences exist in terms of how clubs run/invest in their academies, and participation at a given academy can have an impact on a player's professional career. If this was not the case, smaller clubs would not invest in their youth academies, but resort to poaching rejected talent from larger clubs. We can see that clubs like Arsenal, West Ham, and Leeds United adhere to the sound reputation of their youth programs based on the results from this analysis. Players that attended Leeds United youth training and appeared in the firs team, as opposed to Sheffiel Wednesday, decrease their exit probability from the top leagues by anywhere from $49 \%$ to $64 \%$. Therefore, quality of youth training varies by club and a player's choice of club (if there is a choice) at youth level can have a significan effect on his career length as a professional. Furthermore, the results indicate that clubs like Arsenal, west Ham, and Leeds United have historically outperformed the other clubs in this study in terms of evaluating the ability and potential of their youth products succeeding at the top-level of European soccer. Essentially, the finding suggest that certain clubs do a better job in preparing their players for an extended presence at the top level of European soccer. The importance of club participation at youth level is emphasized even when 
individual-level heterogeneity is accounted for, which should capture the unobserved differential in talent among the players in the data set.

\section{Results for Other Covariates}

Other than in the benchmark model, the position dummies are significan (at 0.01 level of significance in all specification indicating that the position played can play a role on career duration of professional soccer players. It is not surprising that the career duration of goal tenders is significantl longer than for any other position, and these results are consistent with the Frick, Pietzner, and Prinz's (2007) finding for German Bundesliga players. It is quite common for goalkeepers to play well into their late thirties and early forties, which is a rarity for players in other positions. Goalkeepers experience less intense training and physical strain in games, which allows them to preserve their bodies and extend their career. Forwards tend to have the shortest career duration, which is also not surprising considering the scrutiny they face and attention they receive due to the fact their productivity is easily measured and monitored via obvious measures like goals scored. One has to be careful interpreting these results because a player's ability to adapt to other positions is not accounted for. International appearances are signif cant in all specifications indicating that making one additional international appearance reduces a player's exit probability by around $2 \%$. This result appears reasonable because players making international appearances are usually the cream of the crop from their respective countries and are most likely to fin firs team opportunities in the top leagues across Europe. 
Granted, this might not always be the case since playing on the national teams of Northern Ireland or Wales is much easier to accomplish than playing for England. In general, players making international appearances for any country are consistent top league participants. Average season appearances also has a predictable and highly significan negative effect on the hazard rate in all specifications Age of entry has a strong positive impact on the hazard rate ranging from an increase in exit probability of $15 \%$ to $31 \%$ for an additional year added to the age of entry compared to the average player (Note that the average age of entry is 18.6 in this data set). Year of entry, a variable that can indirectly capture institutional changes in English soccer, has a negative effect on the probability of exit for each additional year in M2, M3, and M5. However, the statistical significanc (two out of the four spline terms are not significant in M3 and M5 is not sufficien to make any reliable inference about the effect of the covariate. The results from M2 suggest a slight negative effect on the hazard rate meaning that players entering during the latter years in this study have longer expected career duration. This result weakly suggests that many of the institutional changes (i.e. league changes that increased the number of substitutions allowed in a game and increased the number of teams in the top league) in England over the years, increasing the number of places and possibilities for players in the top tier, outweighed the increasing competitive forces. 


\section{Model Selection}

Model specificatio is improved by allowing for nonlinear functional forms for the average appearances and the year of entry variables. Comparing specification M2 and M3 indicates that allowing for nonlinear functional form of the above variables has a significan effect on the magnitude of youth team coefficien estimates (and the resulting hazard ratios) for most clubs, with West Brom and Southampton being least affected. A similar observation can be made when comparing the results from the models with unobserved heterogeneity, M4 and M5, by looking at the position dummies. The productivity measure covariates seem to be most robust across specifications The presence of individual-level unobserved heterogeneity points to other factors that might be important for a player's survival. Some of these factors might be a player's wages and/or transfer fees paid during his career. However, individual talent and tactical knowledge of a particular player is difficul to measure and should provide a certain degree of unobserved heterogeneity. In terms of loglikelihood, model specificatio is greatly improved by adding productivity variables and nonlinear functional forms to the benchmark model. Accounting for unobserved heterogeneity to M2 and M3 specification also results in an improvement, but not to as great an extent as the previous addition of productivity variables. ${ }^{37}$ It is interesting that the M3 specificatio without heterogeneity outperforms, although not to a great extent, the M4 frailty specificatio in terms of log-likelihood, suggesting that mod-

\footnotetext{
37 This is especially the case for the M3 and M5 models where the difference in log-likelihood is
} almost negligible. 
eling nonlinearity contributes more proportionally than accounting for unobserved heterogeneity. The same observation can be reached by comparing the AIC and BIC for the fve specifications with M5 slightly outperforming M3. The Gompertz distribution shape parameter $\gamma$ is larger in the frailty models (M4 \& M5) than in the reference models (M2 \& M3), meaning that the baseline hazard slopes upwards to a greater extent in models with unobserved heterogeneity. The estimated parameter $\theta,{ }^{38}$ a measure of heterogeneity, is 0.342 and 0.217 for the M4 and M5 specifications respectively, and statistically significan at the 0.01 level. Less unobserved heterogeneity in a model is preferred (as is the case for M5), which is additional evidence that the models allowing for nonlinear functional forms of covariates result in an improvement in specificatio and are preferred to the models that have linear functional form for the relevant covariates (M4). Additionally, the less heterogeneity in a model allows for more appropriate interpretation of any observed duration dependence. ${ }^{39}$ Therefore, the M3 specificatio serves as the best model for interpretation of the estimated hazard ratios considering the almost negligible difference in log-likelihood from M5.

Figure 1.5 (B) graphically displays the estimated hazards of the various models versus the empirical hazard. M2 and M3 slightly overpredict the empirical hazard for the most part but they model the slope closely, especially M3. Models with unob-

\footnotetext{
$38 \theta$ is define as the variance of the gamma distribution for unobserved heterogeneity.

39 One has to be careful in the presence of frailty, as the interpretation in terms of hazard ratios is lost because the proportional effect of a given regressor is no longer constant and independent of survival time.
} 
served heterogeneity, M4 and M5, scale up the estimated reference models (M2 and M3) resulting in significan overprediction of the empirical hazard, especially M4. The baseline hazards for the Gompertz specification are presented in Figure 1.5 (A). The shape of the baseline hazards for the parametric specification accurately model duration dependence, suggesting there is positive state dependence. There is no indication that the Gompertz model is mis-specified the underlying time dependency seems to be properly characterized, which will generally result in more precise estimates than the ones produced by semi-parametric and nonparametric models where the underlying time-dependency is left unspecified This hypothesis will be tested by choosing a Cox model with a fl xible baseline in the following sub-section.

\subsubsection{Model Evaluation}

The Cox proportional hazard semiparametric model is estimated, and reported in Table 1.7, as an alternative to the Gompertz parametric model, and the solid youth training reputation of clubs like Arsenal, West Ham, and Leeds United is upheld in the Cox PH model. M3 outperforms the other specification in terms of modeling duration dependence and following the empirical hazard, which is evident in Figure $1.6(\mathrm{~A}, \mathrm{~B})$.The Therneau and Grambsch nonproportionality test ${ }^{40}$ suggests that the $\mathrm{PH}$ assumption is violated in M2 and M3, both globally and at individual covariate

40

$$
T_{k}=\frac{\left\{\sum\left(g_{k}-\bar{g}\right) s_{k}^{*}\right\}^{2}}{d I_{k} \sum\left(g_{k}-\bar{g}\right)^{2}}
$$

where $I_{k}$ is the information matrix where elements for covariate $k$ and $d$ are the event times. The plot of Schoenfeld residuals against time should display a zero slope if the residual exhibits a unsystematic pattern at each failure time. 
level. In addition, the goodness of fi test for M3, which is displayed in Figure 1.7 (A), based on Cox-Snell residuals suggests that the Cox model does not fi the data well. The idea is that if the Cox PH model fit the data, then these residuals should be distributed unit-exponentially. Plotting the integrated hazard based on these residuals against the hazard rate estimates backed out of the Cox model should result in a 45 degree slope if the Cox specificatio is appropriate; The divergence from the 45 degree line midway suggests that the Cox model does not fi the data very well.

The violation of the PH assumption and the unsatisfactory fi of the Cox semiparametric model, coupled with the reasonable estimation of duration dependence by the Gompertz model, suggest that the parametric model provides a better specificatio and fi of the data in this analysis. In selecting the most appropriate model preference is given to the slope of the estimated hazard because it may be used to identify true duration dependence. Figure 1.7 (B) contains the estimated hazards for the M3 Cox and Gompertz specification against the empirical hazard. Even though the estimated hazards for the two competing models are very close, the Gompertz model seems to be more efficien (varies less with the data) for the large portion of the examination period. With respect to duration dependence, the Gompertz model is the better predictor as it mimics the slope of the empirical hazard more closely than the Cox semiparametric model. In addition to visual inference about the slope and efficien y of the estimated hazards, Vuong's test for discriminating between rival non-nested models is performed, using the following hypotheses: 
1. The two distributions are equal.

2. $H_{c}$ is better than $H_{g}$.

3. $H_{c}$ is worse than $H_{g}$.

The Vuong test statistic is simply the normalized ratio of the rival models' average log-likelihoods. The details of the Vuong test are explained in the appendix. The calculated Vuong statistic is sufficientl large and negative ${ }^{41}$ indicating that the Gompertz parametric model is closer to the true specificatio than the Cox model. This test confirm the intuition from the above results and leads us to rely on the Gompertz model to make inference on the effect of youth training on career duration of professional soccer players.

\subsection{Home-Team Spell Duration Results}

This section discusses the results from the analysis of individual player spell duration at his home club (the club that trained the player). The main objective here is to assess which clubs tend to rely on their youth systems more heavily in terms of their firs team composition. None of the parametric models considered seem appropriate. AIC scores suggest that the Generalized Gamma Model has the most desirable properties, but further tests indicate that none of the nested models are satisfactory

41 The calculated Vuong statistic $=-718$ and is statistically significan at all conventional critical values for the normal distribution (i.e. $-1.96=0.05$ level of significance) The high negative value suggests that the alternative hypothesis (look in the appendix) is true. 
for this analysis. In addition, the baseline hazards for the estimated Gamma specifi cations suggest that duration dependence is poorly estimated.

\subsubsection{Semiparametric Model}

Once again, three different specification are estimated for the Cox PH spell duration model: M1: initial conditions only, M2: initial conditions and productivity measure variables, M3: full model including initial conditions, productivity measures and nonlinear functional form for certain covariates.

The results of the estimations are presented in Table 1.8. The results are explained in terms of M3 because, as it will be explained subsequently, this specifi cation is clearly superior to the other two. Two thirds (10 out of 15) of the youth team regressors are statistically significan at conventional levels. This analysis outlines the differences in players firs team participation rates at their home teams. The results suggest that certain clubs are more reliant than others on their youth academies in terms of their firs team composition. However, it is plausible that mediocre players chances to move from lower to higher ranked teams is more restricted than mediocre player movement in the opposite direction. Shorter spells at top clubs are to be expected for quality constrained players, whereas their probability of survival at lower ranked clubs is more likely to be higher. Therefore, it is hard to interpret whether clubs in this analysis rely more or less on their youth system because of choice or necessity due to financial/reputatio constraints. Clubs like Arsenal, West 
Ham and Leeds United seem to maintain the strongest bond with the players they train at youth level. The spell analysis effect is similar to the career duration effect for these clubs, meaning that they not only produce the most durable players overall, but tend to keep them at their parent club the longest as well. Arsenal has a history (particularly in the last 15-20 years) of producing and maintaining quality players from their youth program in their firs team, and they seem to be one of the most competitive and financiall stable clubs in England in the recent era. ${ }^{42}$ On the other hand, a club like Everton, that has the longest history of appearances in the top tier of English soccer, seems to be on the low end of the statistically significan youth team regressors for this analysis, but the career duration of its youth trainees is in the upper third of the clubs examined. This suggests that they haven't been able or willing to hold on to their youth products for a sufficientl long period of time compared to some of the other teams in the analysis. This might not necessarily be a bad thing for the club because they might have cashed in from the sale of a these players in the transfer market. ${ }^{43}$ It is not surprising that the expected spell duration is larger for some of the smaller clubs (i.e. West Ham, Leeds United, and Southampton) in this analysis versus some larger clubs (i.e. Manchester United or Liverpool). Naturally, it is much more difficul for youth trainees to establish themselves at larger clubs like Manchester United due to the more competitive firs team environment. Smaller

42 The current Arsenal manager, Arsene Wenger, has developed a strong youth program at the club and has emphasized relying on home grown talent versus active purchasing in the transfer market. In fact, Arsenal were one of the few English clubs in recent years to report profit instead of losses.

43 A recent example is Wayne Rooney who was sold to Manchester United. 
clubs that are not as financiall endowed have to rely on domestic youth products in order to remain competitive. Therefore, loyalty is not the only factor to consider when interpreting the results of this analysis. Larger clubs might create the same or even higher degree of loyalty with their youth players, but their financia strength and ambitions make it much more difficul for youth players to break into the firs team and remain there for an extended period of time. Hence, the difference may be that smaller clubs are more reliant on their youth system due to their financia inability to compete for top talent in the transfer market. In addition, the notion that these smaller clubs are better known for their youth academies than their professional teams might imply a signaling effect on their behalf. This would imply that these HT duration results might be less a factor of youth training, but rather more to do with a signal that these clubs want to send and maintain their reputation.

Except for wing players, all of the position coefficient are highly significant The goaltenders tend to have the longest expected duration with their home team, while the forwards (benchmark), unsurprisingly, have the shortest expected home spell duration compared to the other positions. A proxy for productivity, ${ }^{44}$ average appearances per season, has a strong and expected negative effect on the hazard rate probability or positive impact on expected spell duration. Age of entry has a strong and expected negative effect on expected spell duration. Year of entry has a statistically significan negative effect on expected spell duration. This suggests that players

\footnotetext{
44 International appearances and average goals per season are counted for the player's entire career
} and are not available for this spell analysis. 
entering the professional soccer market in the latter years of this study have a reduced probability of survival in the top leagues playing for their home team than players in the past. This seems like a plausible result considering that institutional changes in England and Europe have made the domestic soccer markets more integrated and competitive over the years of this study, making it that much harder for players to maintain their places in the firs team. These changes would subject players to more movement in the market and, as a result, shorter expected spell durations during their career. It is interesting that this covariate has opposing effects for career and spell duration results. This is not surprising considering that institutional changes increased the number of possibilities for players to remain in the top leagues, but at the same time the enhanced competitive nature of the market subjected the players to more movement within the market. One has to be careful in interpreting this variable because the majority of the players in the data base were not affected by the changes arising as a result of the Bosman ruling, which has significantl altered the European soccer environment.

In terms of log-likelihood, M2 and M3 are significan improvements on the benchmark specificat on, M1. The proportionality of hazards assumption is violated for M2, both globally and locally for the appearances per season covariate. However, the PH assumption holds up with a high level of significanc for M3, both globally and locally for each covariate. As mentioned earlier, the nonproportionality test detects a number of other specifi ation errors (including nonlinearity of covariates) and 
this appears to be evident here, as M2 appears to be mis-specified In addition, the strong evidence that the $\mathrm{PH}$ assumption holds indicates that the variance of unobservables is negligent and that heterogeneity does not pose an empirical threat to the estimation results. This assumption is further solidifie by the goodness of fi test results. Figure 1.8 $(\mathrm{A}, \mathrm{B})$ provide further evidence that $\mathrm{M} 3$ is superior to the other two specification in terms of modeling duration dependence and model fit The satisfactory goodness of fi test using Cox-Snell residuals for M3 is presented in Figure 1.9. ${ }^{45}$ The test and the resulting graph verify the statements made above about the quality of Cox model specificatio for this spell duration analysis.

\subsection{Endogeneity Testing}

A split sample test is used to test if the model specification for both career durations and home team spell duration suffers from endogeneity bias ${ }^{46}$, which is particularly of interest for the proxies used for player productivity. The test follows the same steps as the one proposed by Huynh, Petrunia and Voia (2010):

1. Randomly split the sample of soccer players in two equal parts.

2. Estimate the career and home team duration models using the covariates $\left(x^{(1)}\right)$ from the firs sample and retrieve the estimated coefficient $\left(\hat{\beta}_{1}^{(1)}\right)$.

45 The 45 degree slope is closely matched by the integrated hazard based on these residuals.

46 We consider Model 3 as the tested model as it was the model that performed better on predicting the home team and career durations. 
3. Use the estimates from sample one, $\hat{\beta}_{1}^{(1)}$, and the sample two information $\left(x^{(2)}\right)$ to create predicted durations, then with the data from sample two, generate a variable which is the difference between the actual and the predicted durations: $\log \left(t^{(2 *)}\right)=\log \left(t^{(2)}\right)-x^{(2)} \hat{\beta}_{1}^{(1)}$.

4. The new outcome variable $\log \left(t^{(2 *)}\right)$ is then regressed against the same covariates from the two models using the data from sample two: $\log \left(t^{(2 *)}\right)=$ $-x^{\prime(2)} \beta_{1}^{(2)}+\log \left(u^{(2)}\right)$

5. A $\chi^{2}(m)$ test is constructed, where $m$ is the number of variables, with the null hypothesis of no bias or $H_{0}: \beta_{1}^{(2)}=0$. If there is a systemic bias induced by the unobserved heterogeneity assumption, the test statistic would be rejected.

More details about the motivation and constrained of this test statistic can be found in the appendix. Note that the same test is used for both duration problems as no formal test can be used for the different parametric and semiparametric duration models. This test however can give an idea of what variables are potential candidates to be endogenous given that the test is performed on an approximately equivalent linear duration model specification This linear specificatio can be viewed as an upper bound for the endogeneity bias. As the two preferred models are nonlinear, the effect of the potential endogeneity bias will be lower than in the tested model. The results of the split sample tests are presented in Table 1.9. The results of the test 
suggest that career duration model suffers from less bias than the home team duration model and that the sources of bias may be found in some of the home team dummies.

\subsection{Conclusion}

Little is known in economic literature about the impact of education/training at the early stages of an athlete's career on his survival as a professional, and this chapter is the firs attempt to address this topic to a certain extent. ${ }^{47}$ A unique data set of English trained (at one of the 16 clubs examined) soccer players is used to estimate their career and spell duration. One contribution of this study is that information on youth training program participation is included in the duration models, and local effects are estimated that outline the differences in players careers associated with emergence from the youth academies examined. The results of model estimation indicate that duration varies by club, which might suggest that the different training methods employed and ability to assess talent at youth academies play an important role in the longitude of a player's career. The finding indicate that certain clubs that are renowned for their youth academies outperform other clubs in the study in terms of career duration for the players that come through their youth system. One would expect that median youth trainees from more successful clubs should outlast their counterparts from less successful clubs at the top level of European soccer. However, the results indicate that this is not the case, suggesting that training and talent

47 We note that the analysis in this paper is not fully representative, as only a specifi subsample of youth trainees is selected based on their participation at the professional level. 
scouting differ significantl among soccer clubs and smaller clubs have the ability to outperform or compete with larger clubs by investing in their youth academies. The spell duration results suggest that some smaller clubs outperform larger clubs in terms of producing and holding on to home-grown talent, which would be a necessary condition for them to remain competitive in light of their lagging financia resources that limit their transfer market activity.

One limitation of the data is that the analysis is able to measure only local effects of these youth academies, rather than general. It would be interesting to look at general effects, pending data availability, of European youth academies and include lower ranked clubs in the analysis. ${ }^{48}$ Data on duration and intensity of training at particular academies could enhance the analysis of their general effects. Additionally, assessing the impact of the Bosman ruling on these youth academies, and clubs' subsequent investment in them, would be a worthwhile exercise. One would expect that larger and more successful clubs benefite more from the increased talent pool available after the ruling, due to their superior resources available, and that the production of top-level players started favoring these larger clubs. Furthermore, similar studies can be performed on other European soccer leagues, and other sports that have similar structures. In addition, relevant data for manager turnover can be additionally

48 In general, we would expect that lower ranked clubs serve as feeder clubs to larger top-division clubs. Their most talented players are likely to leave for larger clubs at the firs chance they get (perhaps at 16 when the 90 minute rule is no longer in effect) and it would be debatable which club is to be credited with the player's youth development in case he makes it as a top-level professional. 
collected to examine its effect on player duration, as this could be an important factor in any player's career. 


\subsection{Appendix}

\subsubsection{Web Sites Used in Data Collection}

Table 1.1: Data Collection Web Sites

\begin{tabular}{l}
\hline Website \\
\hline http://www.lerwill-life.org.uk/astonvilla/ \\
http://www.astonvillaplayerdatabase.com \\
http://www.statbunker.com/ \\
http://www.leeds-fans.org.uk/leeds/ \\
http://www.lfchistory.net/Players/ \\
http://www.mcfcstats.com/ \\
http://www.westhamstats.info/westham.php?west=0 \\
http://www.adrianbullock.com/swfc/stats/swfcarch.htm \\
http://www.toon1892.co.uk/ \\
http://www.sporting-heroes.net/football/ \\
http://stats.football365.com/hist/default.html \\
http://www.soccerbase.com/
\end{tabular}

\subsubsection{Parametric Models}

One of the limitations of parametric models is that one has to make an assumption about the shape of the baseline hazard rate, i.e. the shape of the underlying duration dependence. Hence, parametric models can suffer from mis-specificatio if the baseline hazard places overly strong restrictions (parametric assumptions) on the direction and shape of the duration dependence. On the other hand, if the characterization of the underlying duration dependence is accurate, the parametric models will provide more reliable and accurate estimates than semiparametric or nonparametric models. 
A large number of parametric models are considered, but results are reported only for the Gompertz model for career duration. Akaike's Information Criterion (AIC) is used to distinguish between parametric models. ${ }^{49}$

Table 1.2: Duration Models Considered

\begin{tabular}{llll}
\hline Model & $S(t)$ & $S_{\theta}(t)$ & $h(t)$ \\
\hline Weibull PH (G) & $e^{-e^{x \beta} t^{p}}$ & $\left(1+\theta e^{x \beta} t^{p}\right)^{-\frac{1}{\theta}}$ & $e^{x \beta} p t^{p-1}$ \\
Weibull AFT (G) & $e^{-e^{-p x \beta} t^{p}}$ & $\left(1+\theta e^{-x x \beta} t^{p}\right)^{-\frac{1}{\theta}}$ & $e^{-p x \beta} p t^{p-1}$ \\
Gompertz PH (G) & $e^{\frac{e^{-x \beta}}{\gamma}\left(1-e^{\gamma t}\right)}$ & $\left(1-\theta \frac{e^{x \beta}}{\gamma}\left(1-e^{\gamma t}\right)\right)^{-\frac{1}{\theta}}$ & $e^{x \beta+\gamma t}$ \\
Gompertz AFT (G) & $e^{\frac{e^{-x \beta}}{\gamma}\left(1-e^{\gamma t}\right)}$ & $\left(1-\theta \frac{e^{-x \beta}}{\gamma}\left(1-e^{\gamma t}\right)\right)^{-\frac{1}{\theta}}$ & $e^{-x \beta+\gamma t}$ \\
Cox PH (G) & $e^{-e^{x \beta} \Lambda_{0}(t)}$ & $\left(1+\theta e^{x \beta} \Lambda_{0}(t)\right)^{-\frac{1}{\theta}}$ & $e^{x \beta} \lambda_{0}(t)$ \\
Weibull PH (IG) & $e^{-e^{x \beta} t^{p}}$ & $e^{\frac{1}{\theta}\left[1-\left(1+2 \theta e^{x \beta} t^{p}\right)^{\frac{1}{2}}\right]}$ & $e^{x \beta} p t^{p-1}$ \\
Weibull AFT (IG) & $e^{-e^{-p x \beta} t^{p}}$ & $e^{\frac{1}{\theta}\left[1-\left(1+2 \theta e^{-p x \beta} t^{p}\right)^{\frac{1}{2}}\right]}$ & $e^{-p x \beta} p t^{p-1}$ \\
Gompertz PH (IG) & $e^{\frac{e^{x \beta}}{\gamma}\left(1-e^{\gamma t}\right)}$ & $e^{\frac{1}{\theta}\left[1-\left(1-2 \theta \frac{e^{x \beta}}{\gamma}\left(1-e^{\gamma t}\right)\right)^{\frac{1}{2}}\right]}$ & $e^{x \beta+\gamma t}$ \\
Gompertz AFT (IG) & $e^{\frac{e^{-x \beta}}{\gamma}\left(1-e^{\gamma t}\right)}$ & $e^{\frac{1}{\theta}\left[1-\left(1-2 \theta \frac{e^{-x \beta}}{\gamma}\left(1-e^{\gamma t}\right)\right)^{\frac{1}{2}}\right]}$ & $e^{-x \beta+\gamma t}$ \\
\hline
\end{tabular}

Note: (G) denotes a Gamma distribution, $g(v)=\frac{v^{\frac{1}{\theta} \square 1} e^{\frac{\square v}{\theta}}}{\Gamma\left(\frac{1}{\theta}\right) \theta^{\frac{1}{\theta}}}$, with a mean of unity. The population hazard for this case is define as $h_{\theta}(t)=h(t)(1-\theta \ln (S(t)))^{-1}$. (IG) denotes an Inverse Gaussian distribution, $g(v)=\left[\frac{1}{2 \pi \theta v^{3}}\right]^{\frac{1}{2}} e^{-\frac{1}{2 \theta}\left(v-2+\frac{1}{v}\right)}$, with a mean of unity. The population hazard for this case is define as $h_{\theta}(t)=h(t)(1-2 \theta \ln (S(t)))^{-\frac{1}{2}}$. The unconditional density function in both cases is $f_{\theta}(t)=h_{\theta}(t) S_{\theta}(t)$.

\subsubsection{Semiparametric Models}

Semiparametric models, like the Cox Proportional Hazards (PH) model, provide alternatives to parametric models. These models are more general and allow the estimation of slope parameters for the covariates irrespective of what the baseline hazard

49 AIC is a measure of the relative quality a statistical model, and is often used for selection among competing models. The most appropriate model minimizes the Kullback-Leibler distance between the model and the data. The actual measure deals with the trade-off between goodness of fi and complexity of the model. 
looks like. As such, the Cox PH model makes no assumptions about the distribution of survival times and is robust to mis-specificatio of the baseline hazard. A typical semiparametric model is of the form:

$$
h_{i}\left(t_{i} \mid x_{i}, v_{i}\right)=\phi\left(x_{i}\right) \lambda\left(t_{i}\right)
$$

where $\phi\left(x_{i}\right)=\exp \left(x_{i} \beta\right)$ is a function of the observable time-invariant covariates, and $\lambda\left(t_{i}\right)$ is the nonparametric baseline hazard for individual player $i$.

The Cox model assumes that the covariates will have a proportional and constant effect that is invariant to time. According to Box-Steffensmeier and Jones (2004), testing the proportionality of hazards assumption is arguably the primary concern when fittin a Cox model. Residual-based tests using Schoenfeld residuals $^{50}$ are particularly useful when testing the proportional hazards assumption. The Therneau-Grambsch test (1994) is based on scaled Schoenfeld residuals and is applicable both globally and at specifi covariate level. ${ }^{51}$ One has to be careful when interpreting the results because the proportionality of hazards test can yield a false result of nonproportionality if the model is mis-specifie (Therneau and Grambsch 1994, 2000). Therefore, attempting to specify the model correctly should precede

50 If the residual exhibits a unsystematic pattern at each failure time, then this suggests that the covariate effect is not changing with time so the $\mathrm{PH}$ assumption holds. In this case, the plot of Schoenfeld residuals against time should display a zero slope.

51 The test statistic for the nonproportionality test is:

$$
T_{k}=\frac{\left\{\sum\left(g_{k}-\bar{g}\right) s_{k}^{*}\right\}^{2}}{d I_{k} \sum\left(g_{k}-\bar{g}\right)^{2}}
$$

where $I_{k}$ is the information matrix where elements for covariate $k$ and $d$ are the event times. 
any testing of the $\mathrm{PH}$ assumption. If the $\mathrm{PH}$ assumption fails $\mathrm{s}^{52}$ the most common remedy is to interact the covariate that failed the $\mathrm{PH}$ test with the natural log of time and include both (the covariate and the time interaction term) in a new regression.

\subsubsection{Vuong Test for Comparing Two Non-Nested Models}

Vuong (1989) proposed a test ${ }^{53}$ for selecting between two non-nested models. This test is useful in selecting the more appropriate model for the career duration analysis of this chapter, specificall deciding between the parametric Gompertz model versus the semiparametric Cox model. Using the individual densities for the models of interest, we construct the average log-likelihoods for the two rival models. Assuming $H_{c}$ and $H_{f}$ represent the Cox and Gompertz cumulative densities, respectively, the null hypothesis (model equivalence) of Vuong's test is:

$$
H_{0}: E^{0}\left[\ln \frac{c\left(t \mid X_{i} ; \theta_{*}\right)}{g\left(t \mid X_{i} ; \gamma_{*}\right)}\right]=0
$$

The alternative hypotheses are:

$$
H_{c}: E^{0}\left[\ln \frac{c\left(t \mid X_{i} ; \theta_{*}\right)}{g\left(t \mid X_{i} ; \gamma_{*}\right)}\right]>0
$$

52 The $\mathrm{PH}$ assumption needs to pass the test globally and at the individual covariate level for the researcher to be able to rely on the model's assumption.

53 The test makes use of the Kullback-Leibler information criteria (KLIC):

$$
K L I C=E^{0}\left[\ln h^{0}\left(Y_{t} \mid X_{t}\right)\right]-E^{0}\left[\ln f\left(Y_{t} \mid X_{t} ; \theta_{*}\right)\right]
$$

where $h^{0}()$ is the true conditional density of $Y_{t}$ given $X_{t}$, and $\theta_{*}$ are the pseudotrue values of $\theta$ (the estimates of $\theta$ when $f\left(Y_{t} \mid X_{t}\right)$ is not the true model). 


$$
H_{g}: E^{0}\left[\ln \frac{c\left(t \mid X_{i} ; \theta_{*}\right)}{g\left(t \mid X_{i} ; \gamma_{*}\right)}\right]<0
$$

that ascertain that $H_{c}$ is better or worse than $H_{g}$, respectively.

The expected value in the above hypotheses is unknown, but Vuong demonstrates that under fairly general conditions:

$$
\frac{1}{n} L R_{n}\left(\hat{\theta}_{n}, \hat{\gamma}_{n}\right) \stackrel{a . s .}{\longrightarrow} E^{0}\left[\ln \frac{c\left(t \mid X_{i} ; \theta_{*}\right)}{g\left(t \mid X_{i} ; \gamma_{*}\right)}\right]
$$

meaning that the expected value can be consistently estimated by $\left(\frac{1}{n}\right)$ times the likelihood ratio statistic. The actual test is then:

$$
\begin{gathered}
\text { under } H_{0}: \frac{L R_{n}\left(\hat{\theta}_{n}, \hat{\gamma}_{n}\right)}{(\sqrt{n}) \stackrel{D}{\longrightarrow}} N(0,1) \\
\text { under } H_{c}: \frac{L R_{n}\left(\hat{\theta}_{n}, \hat{\gamma}_{n}\right)}{(\sqrt{n}) \hat{\omega}_{n}} \stackrel{D}{\longrightarrow}+\infty \\
\text { under } H_{g}: \frac{L R_{n}\left(\hat{\theta}_{n}, \hat{\gamma}_{n}\right)}{(\sqrt{n}) \hat{\omega}_{n}} \stackrel{D}{\longrightarrow}-\infty
\end{gathered}
$$

where

$$
L_{n}\left(\hat{\theta_{n}}, \hat{\gamma}_{n}\right) \equiv L_{n}^{c}(\hat{\theta})-L_{n}^{g}(\hat{\gamma})
$$

and

$$
\hat{\omega}_{n} \equiv \frac{1}{n} \sum_{i=1}^{n}\left[\ln \frac{c\left(t \mid X_{i} ; \theta_{*}\right)}{g\left(t \mid X_{i} ; \gamma_{*}\right)}\right]^{2}-\left[\frac{1}{n} \sum_{i=1}^{n} \ln \frac{c\left(t \mid X_{i} ; \theta_{*}\right)}{g\left(t \mid X_{i} ; \gamma_{*}\right)}\right]^{2}
$$


represents the variance, which is the expectation of the squared difference in the individual log-likelihoods minus the square of the expectation. For the purposes of this study:

$$
\begin{gathered}
H_{c}: H(t)=S(t)+1=\exp \left[\exp (-x \beta) \Lambda_{0}(t)\right]+1 \\
c\left(t \mid X_{i} ; \theta_{*}\right)=h(t) S(t)=\exp (x \beta) \lambda_{0}(t) \exp \left[\exp (-x \beta) \Lambda_{0}(t)\right]
\end{gathered}
$$

for the Cox model, where $\Lambda_{0}$ is the integrated baseline hazard and $\lambda_{0}$ is the baseline hazard, and

$$
\begin{gathered}
H_{g}: H(t)=S(t)+1=\exp \left[\frac{\exp (x \beta)}{\gamma}(1-\exp (\gamma t))\right]+1 \\
g\left(t \mid X_{i} ; \gamma_{*}\right)=h(t) S(t)=\exp (x \beta+\gamma t) \exp \left[\frac{\exp (x \beta)}{\gamma}(1-\exp (\gamma t))\right]
\end{gathered}
$$

for the Gompertz model.

\subsubsection{Split Sample Test}

As no formal theoretical test for endogeneity bias of nonlinear duration models is found in the literature, in this chapter I use an empirical type test based on Dufour and Jasiak (2001) and introduced by Huynh, Petrunia and Voia (2010) to test for potential correlation between observables and unobservables in our career and home team duration analyses. The full details of applying this test are found Huynh, Petru- 
nia and Voia (2010). The intuition behind the test suggest that some semiparametric or parametric models can be expressed as a linear model or an Accelerated Failure Time (AFT) model if a proper time scale $T^{*}=-\log \left(\Lambda_{0}(T)\right)$ is considered. Here the equivalent time scale $-\log \left(\Lambda_{0}(T)\right)$ define the integrated baseline hazard. As the equivalent time scale is not available and needs to be estimated, I rely on the observed time scale to estimate a linear AFT model. ${ }^{54}$ As this is not a perfect equivalence as we rely on the similarities of the estimated models with the proposed AFT model, we take this analysis as informative and not necessary as a perfect test to measure the biases associated to the career and home team duration analyses. However, the linear specificatio of the tested model provides a benchmark for the potential bias in parameters. This can be explained by the fact that the effect of endogeneity may not be alleviated by the functional form specificatio of the model as it may happen with some nonlinear models, where identificatio can be achieved through the functional form specification The reduced form linear AFT model is define as:

$$
\log (t)=-x^{\prime} \beta+\log (u)
$$

where $t$ is the observed duration and $u$ is the error term. Note that the AFT model has the reverse sign of the parameter estimates than the semiparametric and the parametric models used in our analyses. Under this specificatio each coefficien summarizes

54 The Cox proportional hazard model can be expressed as a linear model or an Accelerated Failure Time (AFT) model if a proper time scale, which def nes the integrated baseline hazard, is considered. As the regression parameters for the Cox proportional hazard and the AFT models are often approximately proportional, qualitative inferences should be robust to misspecificatio errors especially when moderate censoring is present in the data. Both the Cox model and semiparametric versions of the AFT model are models that leave the baseline hazard (or, equivalently, the baseline survival distribution) unspecified) For testing purposes, I rely on the similarities of the two models. 
the proportional effect of absolute changes of the covariate on the survivor function, and $\exp (\beta)$ is definin the time ratio. The split sample test is done as follows: Coefficient are broken into parameters of interest $\left(\beta_{1}\right)$ and the nuisance parameters $\left(\beta_{2}\right)$. $\hat{\beta}_{1}^{(1)}$ denotes the estimate for the parameters of interest from the firs sub-sample. The null hypothesis $H_{0}$ concerns the parameters of interest and allows the nuisance parameter to remain unknown. In this case, the null hypothesis is $H_{0}: \beta_{1}=\hat{\beta}_{1}^{(1)}$ or that the coefficient are the same across the split samples. To test $H_{0}$ using the second sample, the estimates from the firs sample are considered as being the true parameters. 


\subsection{Tables and Figures}

Table 1.3: Summary Statistics

\begin{tabular}{lcccc}
\hline \multicolumn{1}{c}{ Variable } & Mean & Std. Dev. & Min. & Max. \\
\hline HT duration & 4.517 & 3.592 & 0 & 19 \\
Total duration & 6.374 & 4.846 & 1 & 21 \\
HT appearances & 82.108 & 112.166 & 0 & 613 \\
Total appearances & 126.616 & 148.904 & 1 & 714 \\
International caps & 4.921 & 14.804 & 0 & 108 \\
Goals & 15.202 & 33.453 & 0 & 366 \\
Age at entry & 18.602 & 1.751 & 15 & 29 \\
Year of entry & 1974.084 & 13.056 & 1946 & 2003 \\
\hline
\end{tabular}

Table 1.4: Hazard Summary for Players' Career and HT Spell Duration

\begin{tabular}{ccccccc}
\hline \multicolumn{4}{c}{ Career Duration } & \multicolumn{3}{c}{ Home Team Spell Duration } \\
\hline time & $\mathbf{n}$ & exits & $\mathbf{h}$ & $\mathbf{n}$ & exits & $\mathbf{h}$ \\
1 & 1121 & 187 & $16.68 \%$ & 1098 & 227 & $20.67 \%$ \\
2 & 934 & 116 & $12.42 \%$ & 871 & 140 & $16.07 \%$ \\
3 & 818 & 122 & $14.91 \%$ & 731 & 164 & $22.44 \%$ \\
4 & 696 & 93 & $13.36 \%$ & 567 & 120 & $21.16 \%$ \\
5 & 603 & 85 & $14.10 \%$ & 447 & 119 & $26.62 \%$ \\
6 & 518 & 70 & $13.51 \%$ & 328 & 70 & $21.34 \%$ \\
7 & 448 & 52 & $11.61 \%$ & 258 & 53 & $20.54 \%$ \\
8 & 396 & 55 & $13.89 \%$ & 205 & 53 & $25.85 \%$ \\
9 & 341 & 44 & $12.90 \%$ & 152 & 35 & $23.03 \%$ \\
10 & 297 & 50 & $16.84 \%$ & 117 & 30 & $25.64 \%$ \\
11 & 247 & 40 & $16.19 \%$ & 87 & 19 & $21.84 \%$ \\
12 & 207 & 45 & $21.74 \%$ & 68 & 19 & $27.94 \%$ \\
13 & 162 & 34 & $20.99 \%$ & 49 & 13 & $26.53 \%$ \\
14 & 128 & 34 & $26.56 \%$ & 36 & 13 & $36.11 \%$ \\
15 & 94 & 31 & $32.98 \%$ & 23 & 8 & $34.78 \%$ \\
16 & 63 & 26 & $41.27 \%$ & 15 & 7 & $46.67 \%$ \\
17 & 37 & 19 & $51.35 \%$ & 8 & 5 & $62.50 \%$ \\
18 & 18 & 7 & $38.89 \%$ & 3 & 2 & $66.67 \%$ \\
19 & 11 & 7 & $63.64 \%$ & 1 & 1 & $100.00 \%$ \\
20 & 4 & 2 & $50.00 \%$ & & & \\
21 & 2 & 2 & $100.00 \%$ & & & \\
\hline
\end{tabular}

Table 1.5: AIC and BIC Scores for Career Duration

\begin{tabular}{l|ll|ll}
\hline & \multicolumn{2}{|c|}{ AIC } & \multicolumn{2}{c}{ BIC } \\
\hline Model & M2 & M3 & M2 & M3 \\
\hline Exponential & 2609 & 2581 & 2749 & 2742 \\
Weibull & 1877 & 1738 & 2023 & 1904 \\
Gompertz & $\mathbf{1 7 6 1}$ & $\mathbf{1 6 8 7}$ & $\mathbf{1 9 0 7}$ & $\mathbf{1 8 5 3}$ \\
Lognormal & 1886 & 1765 & 2032 & 1931 \\
Loglogistic & 1902 & 1748 & 2047 & 1914 \\
Generalized Gamma & 1854 & 1721 & 2005 & 1892 \\
\hline
\end{tabular}


Table 1.6: Gompertz Model Career Duration Results

\begin{tabular}{|c|c|c|c|c|c|}
\hline & M1 & M2 & M3 & M4 & M5 \\
\hline \multicolumn{6}{|c|}{ Initial Conditions } \\
\hline \multicolumn{6}{|c|}{ Personal Characteristics } \\
\hline Age of entry & $1.151^{* * *}$ & $1.199^{* * *}$ & $1.224^{* * *}$ & $1.31^{* * *}$ & $1.292^{* * *}$ \\
\hline Year of entry & $1.004^{*}$ & $.994^{* *}$ & & 1.310 & \\
\hline Year of entry- 0 & & & .954 & & .943 \\
\hline Year of entry-1 & & & $1.122^{* * *}$ & & $1.113^{* * *}$ \\
\hline Year of entry-2 & & & $.925^{* *}$ & & .948 \\
\hline Year of entry-3 & & & $1.083^{* *}$ & & $1.106^{* * *}$ \\
\hline \multicolumn{6}{|l|}{ Youthteam } \\
\hline Arsenal & $.548^{* * *}$ & $.438^{* * *}$ & $.427^{* * *}$ & $.392^{* * *}$ & $.397^{* * *}$ \\
\hline Chelsea & $.703^{* *}$ & $.446^{* * *}$ & $.465^{* * *}$ & $.489^{* * *}$ & $.501^{* * *}$ \\
\hline Everton & $.709^{* *}$ & $.553^{* * *}$ & $.488^{* * *}$ & $.512^{* * *}$ & $.472^{* * *}$ \\
\hline Liverpool & $.564^{* * *}$ & $.507^{* * *}$ & $.451^{* * *}$ & $.426^{* * *}$ & $.403^{* * *}$ \\
\hline Man U & $.688^{* *}$ & $.589^{* * *}$ & $.634^{* * *}$ & $.573^{* * *}$ & $.611^{* *}$ \\
\hline Man City & .787 & .795 & $.739^{*}$ & .772 & .739 \\
\hline Tottenham & $.690^{* *}$ & $.412^{* * *}$ & $.422^{* * *}$ & $.392^{* * *}$ & $.397^{* * *}$ \\
\hline West Ham & $.634^{* *}$ & $.479^{* * *}$ & $.443^{* * *}$ & $.430^{* * *}$ & $.415^{* * *}$ \\
\hline Aston Villa & .896 & $.607^{* * *}$ & $.634^{* *}$ & .751 & $.706^{*}$ \\
\hline Leeds U & $.505^{* * *}$ & $.474^{* * *}$ & $.382^{* * *}$ & $.423^{* * *}$ & $.362^{* * *}$ \\
\hline Newcastle & 1.083 & $.577^{* * *}$ & $.517^{* * *}$ & $.562^{* *}$ & $.502^{* * *}$ \\
\hline Southampton & $.671^{* *}$ & $.618^{* *}$ & $.614^{* *}$ & $.559^{* *}$ & $.567^{* *}$ \\
\hline Nottingham F & $.669^{* *}$ & $.602^{* * *}$ & $.565^{* * *}$ & $.557^{* *}$ & $.543^{* * *}$ \\
\hline West Brom & .799 & $.557^{* * *}$ & $.553^{* * *}$ & $.548^{* *}$ & $.545^{* * *}$ \\
\hline Coventry City & .754 & $.611^{* * *}$ & $.568^{* *}$ & $.592^{* *}$ & $.549^{* * *}$ \\
\hline \multicolumn{6}{|c|}{$\begin{array}{l}\text { Sheffiel Wed - Benchmark } \\
\text { Position }\end{array}$} \\
\hline Forward & 1.188 & $2.314^{* * *}$ & $2.489^{* * *}$ & $2.248^{* * *}$ & $2.418^{* * *}$ \\
\hline Central Defender & 1.035 & $2.076^{* * *}$ & $2.172^{* * *}$ & $2.055^{* * *}$ & $2.151^{* * *}$ \\
\hline Midfielde & .909 & $1.948^{* * *}$ & $1.942^{* * *}$ & $1.728^{* * *}$ & $1.819^{* * *}$ \\
\hline Winger & $1.419^{* *}$ & $2.355^{* * *}$ & $2.348^{* * *}$ & $2.138^{* * *}$ & $1.77^{* * *}$ \\
\hline Full Back & 1.001 & $1.693^{* * *}$ & $1.86^{* * *}$ & $1.606^{* *}$ & $2.256^{* * *}$ \\
\hline \multicolumn{6}{|c|}{$\begin{array}{l}\text { Goalie - Benchmark } \\
\text { Nationality }\end{array}$} \\
\hline Ireland & $.658^{* *}$ & $1.421^{*}$ & $1.479^{*}$ & 1.433 & $1.494^{*}$ \\
\hline Other & .625 & 1.207 & 1.115 & .936 & .992 \\
\hline \multicolumn{6}{|l|}{ UK - Benchmark } \\
\hline \multicolumn{6}{|c|}{ Productivity Measures } \\
\hline International caps & & $.987^{* * *}$ & $.984^{* * *}$ & $.98^{* * *}$ & $.979^{* * *}$ \\
\hline Average goals & & $.967^{*}$ & $.966^{*}$ & $.956^{*}$ & $.960^{*}$ \\
\hline Average apps & & $.878^{* * *}$ & & $.848^{* * *}$ & \\
\hline Average apps- 0 & & & $.236^{* * *}$ & & $.207^{* * *}$ \\
\hline Average apps-1 & & & $.827^{* * *}$ & & $.784^{* * *}$ \\
\hline Observations & 1121 & 1121 & 1121 & 1121 & 1121 \\
\hline$\gamma$ & .094 & .281 & .296 & .397 & .368 \\
\hline$\theta($ variance of $\gamma)$ & & & & $.342^{* * *}$ & $.217^{* * *}$ \\
\hline$\Delta \log L($ vs. M1) & 0 & 545 & 586 & 567 & 594 \\
\hline
\end{tabular}


Table 1.7: Cox Model Career Duration Results

\begin{tabular}{|c|c|c|c|}
\hline & M1 & M2 & M3 \\
\hline \multicolumn{4}{|c|}{ Initial Conditions } \\
\hline \multicolumn{4}{|c|}{ Personal Characteristics } \\
\hline Age at entry & $1.1340^{* * *}$ & $1.0812^{* * *}$ & $1.2004^{* * *}$ \\
\hline Year at entry & 1.00343 & $.99046^{* * *}$ & \\
\hline Year at entry- 0 & & & .95394 \\
\hline Year at entry-1 & & & $1.1019^{* * *}$ \\
\hline Year at entry-2 & & & $.93755^{* *}$ \\
\hline Year at entry-3 & & & $1.0717^{* *}$ \\
\hline \multicolumn{4}{|l|}{ Youthteam } \\
\hline Arsenal & $.56665^{* * *}$ & $.58522^{* * *}$ & $.47278^{* * *}$ \\
\hline Chelsea & $.70153^{* *}$ & $.45799^{* * *}$ & $.52591^{* * *}$ \\
\hline Everton & $.73372^{*}$ & $.69436^{* *}$ & $.53494^{* * *}$ \\
\hline Liverpool & $.59832^{* * *}$ & .73998 & $.53494^{* * *}$ \\
\hline Man U & $.69301^{* *}$ & $.64518^{* * *}$ & $.66539^{* *}$ \\
\hline Man City & .81303 & .80889 & .74790 \\
\hline Tottenham & $.71118^{* *}$ & $.55023^{* * *}$ & $.46121^{* * *}$ \\
\hline West Ham & $.63538^{* *}$ & $.64693^{* *}$ & $.49416^{* * *}$ \\
\hline Aston Villa & .89724 & .80199 & $.69232^{* *}$ \\
\hline Leeds U & $.52293^{* * *}$ & $.71645^{*}$ & $.41984^{* * *}$ \\
\hline Newcastle & 1.0607 & $.71170^{*}$ & $.56546^{* * *}$ \\
\hline Southampton & $.67954^{*}$ & .76599 & $.66030^{* *}$ \\
\hline Nottingham F & $.69793^{*}$ & $.69942^{*}$ & $.60343^{* * *}$ \\
\hline West Brom & .77666 & $.64168^{* *}$ & $.58881^{* * *}$ \\
\hline Coventry City & .76893 & .75977 & $.61448^{* * *}$ \\
\hline \multicolumn{4}{|c|}{ Sheffiel Wed - Benchmark } \\
\hline \multicolumn{4}{|c|}{ Position } \\
\hline Forward & 1.1670 & $1.6565^{* * *}$ & $2.1073^{* * *}$ \\
\hline Central Defender & 1.0335 & $1.7534^{* * *}$ & $1.9165^{* * *}$ \\
\hline Midfielde & .91732 & $1.6255^{* * *}$ & $1.7404^{* * *}$ \\
\hline Winger & $1.3753^{* *}$ & $1.7889^{* * *}$ & $1.9827^{* * *}$ \\
\hline Full Back & 1.0239 & $1.4678^{* * *}$ & $1.6728^{* * *}$ \\
\hline \multicolumn{4}{|c|}{ Goalie - Benchmark } \\
\hline \multicolumn{4}{|c|}{ Nationality } \\
\hline Ireland & $.67423^{* *}$ & 1.2139 & 1.1662 \\
\hline Other & .69349 & 1.3448 & .98457 \\
\hline \multicolumn{4}{|l|}{ UK - Benchmark } \\
\hline \multicolumn{4}{|c|}{ Productivity Measures } \\
\hline Average apps & & $1.13706^{* * *}$ & \\
\hline Average apps- 0 & & & $.30838^{* * *}$ \\
\hline Average apps-1 & & & $.8010^{* * *}$ \\
\hline International caps & & .99768 & $1.0649^{* * *}$ \\
\hline \multicolumn{4}{|c|}{ Variable Interactions with Log(Duration) } \\
\hline Time-intcaps & & & $.97152^{* * *}$ \\
\hline Time-avgapps & & $.88711^{* * *}$ & \\
\hline Observations & 1121 & 1121 & 1121 \\
\hline$\Delta \log L($ vs. M1) & 0 & 752 & 474 \\
\hline
\end{tabular}

Note: ${ }^{*}, * *$ and ${ }^{* * *}$ indicates statistical significanc at the $0.1,0.05$, and 0.01 levels, respectively. 
Table 1.8: Cox Model HT Spell Duration Results

\begin{tabular}{|c|c|c|c|}
\hline & M1 & M2 & M3 \\
\hline \multicolumn{4}{|c|}{ Initial Conditions } \\
\hline \multicolumn{4}{|c|}{ Personal Characteristics } \\
\hline Age of entry & $1.172^{* * *}$ & $1.104^{* * *}$ & $1.206^{* * *}$ \\
\hline Year of entry & $1.014^{* * *}$ & $1.006^{* *}$ & \\
\hline Year of entry- 0 & & & $1.097^{* * *}$ \\
\hline Year of entry-1 & & & $1.127^{* * *}$ \\
\hline \multicolumn{4}{|l|}{ Youthteam } \\
\hline Arsenal & $.675^{* *}$ & $.726^{*}$ & $.557^{* * *}$ \\
\hline Chelsea & .921 & .841 & .778 \\
\hline Everton & .882 & .848 & $.708^{* *}$ \\
\hline Liverpool & $.671^{* *}$ & .928 & $.605^{* *}$ \\
\hline Man U & .770 & .770 & $.662^{* *}$ \\
\hline Man City & 1.108 & 1.009 & .904 \\
\hline Tottenham & .878 & .790 & $.572^{* * *}$ \\
\hline West Ham & .754 & $.696^{* *}$ & $.566^{* * *}$ \\
\hline Aston Villa & 1.001 & .885 & .820 \\
\hline Leeds U & $.670^{* *}$ & .737 & $.494^{* * *}$ \\
\hline Newcastle & 1.245 & .902 & $.730^{*}$ \\
\hline Southampton & $.656^{* *}$ & $.667^{* *}$ & $.595^{* * *}$ \\
\hline Nottingham F & .824 & .854 & $.711^{*}$ \\
\hline West Brom & 1.009 & .843 & .755 \\
\hline Coventry City & .946 & .907 & .807 \\
\hline \multicolumn{4}{|c|}{ Sheffiel Wed - Benchmark } \\
\hline \multicolumn{4}{|c|}{ Position } \\
\hline Central Defender & $.634^{* * *}$ & $.752^{* * *}$ & $.715^{* * *}$ \\
\hline Midfielde & $.721^{* * *}$ & $.832^{*}$ & $.788^{* *}$ \\
\hline Winger & .915 & .836 & .831 \\
\hline Full Back & $.664^{* * *}$ & $.674^{* * *}$ & $.670^{* * *}$ \\
\hline Goalie & $.720^{* *}$ & $.672^{* * *}$ & $.592^{* * *}$ \\
\hline \multicolumn{4}{|c|}{ Forward - Benchmark } \\
\hline \multicolumn{4}{|c|}{ Nationality } \\
\hline Ireland & .889 & 1.314 & 1.128 \\
\hline Other & .733 & 1.127 & 1.095 \\
\hline \multicolumn{4}{|l|}{ UK - Benchmark } \\
\hline \multicolumn{4}{|c|}{ Productivity Measures } \\
\hline Apps-seas & & $1.067^{* * *}$ & \\
\hline Apps-seas-0 & & & $.420^{* * *}$ \\
\hline Apps-seas-1 & & & $.816^{* * *}$ \\
\hline \multicolumn{4}{|c|}{ Variable Interaction with Log-Duration } \\
\hline Observations & 1098 & 1098 & 1098 \\
\hline$\Delta \log L($ vs. M1) & 0 & 331 & 257 \\
\hline
\end{tabular}


Table 1.9: Split Sample Tests

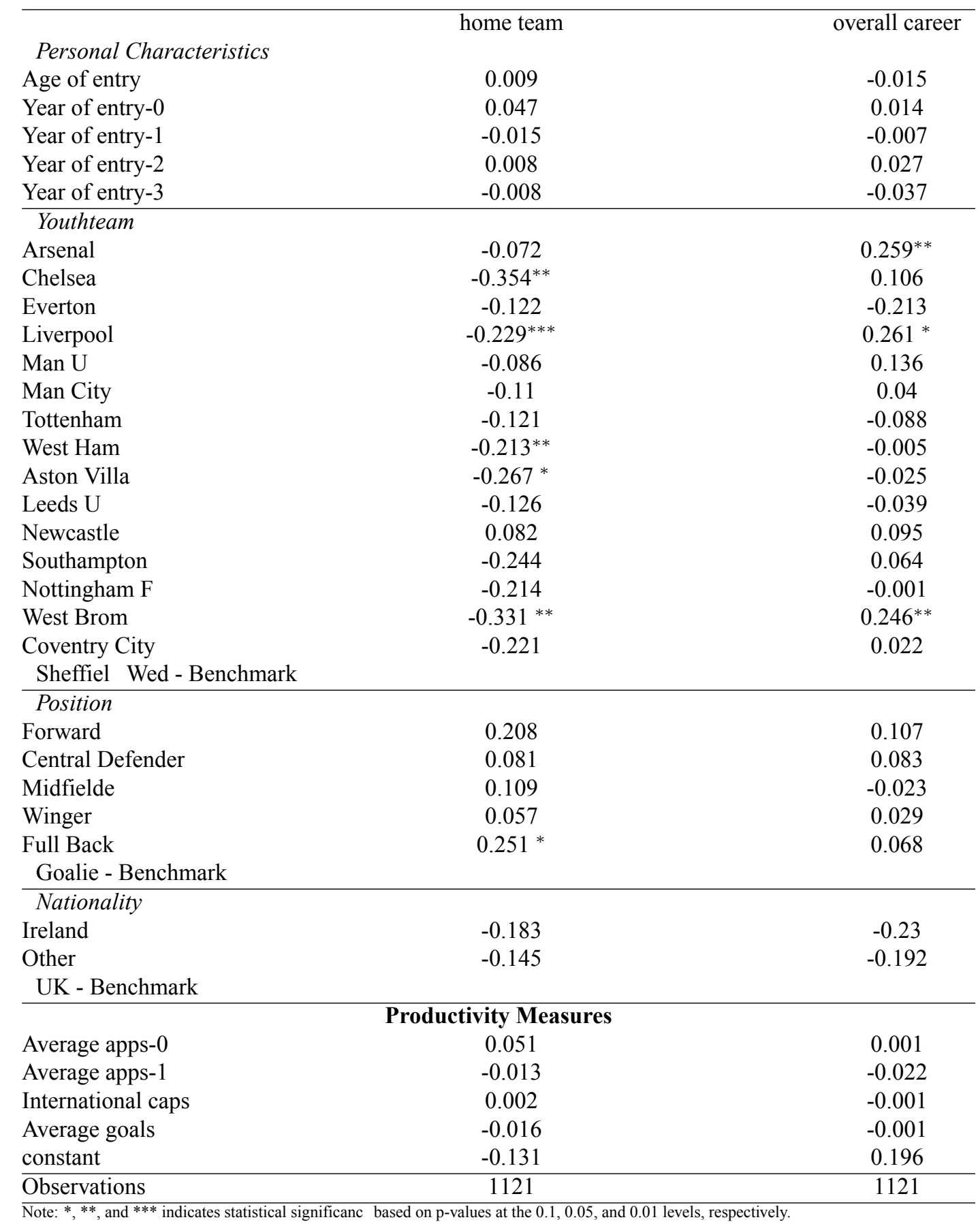




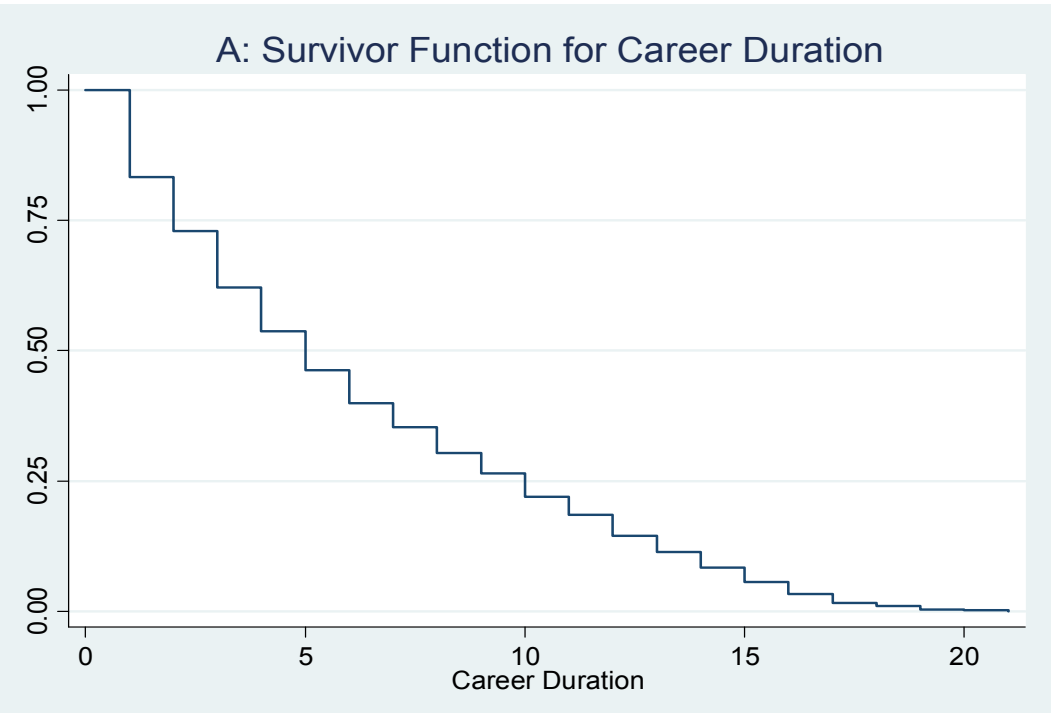

B: Empirical Hazard for Career Duration

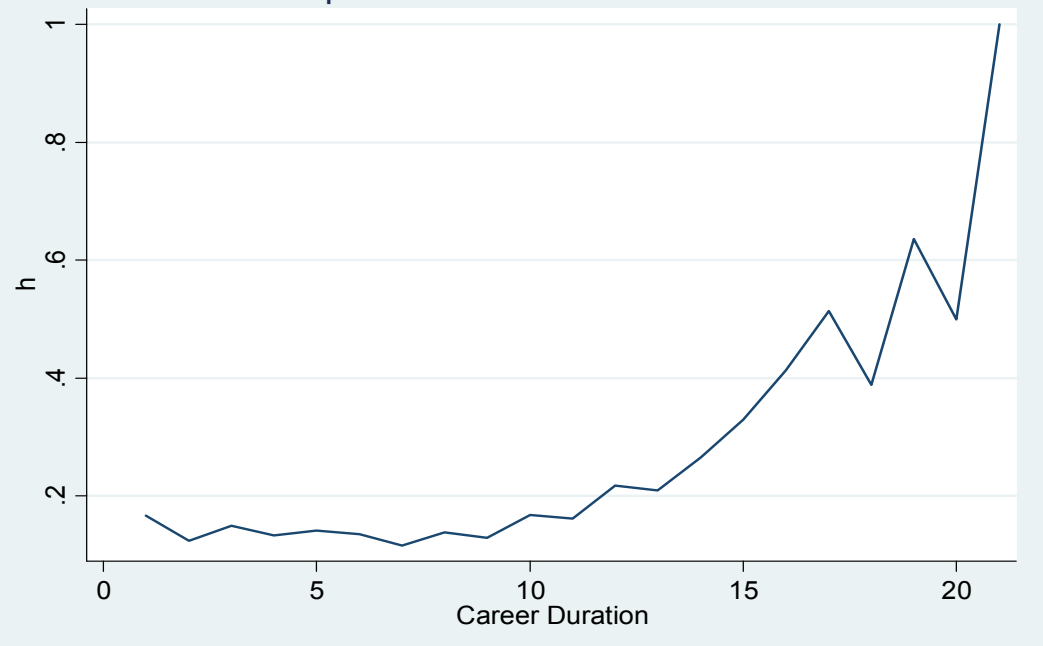

Figure 1.1: (A) Career Duration Empirical Survivor Functiom, (B) Career Duration Empirical Hazard Function 

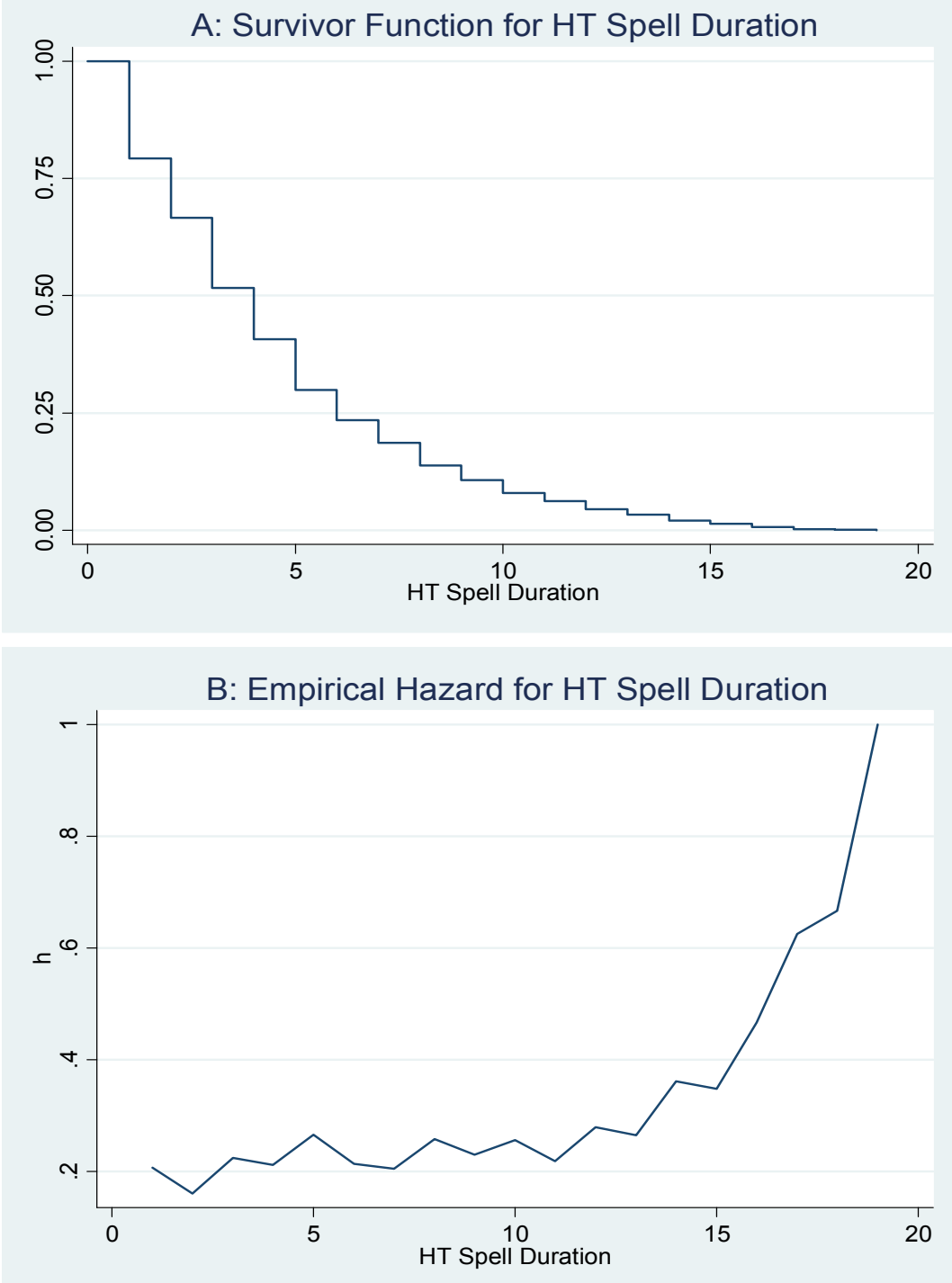

Figure 1.2: (A) HT Spell Duration Empirical Survivor Function, (B) HT Spell Duration Empirical Hazard Function 

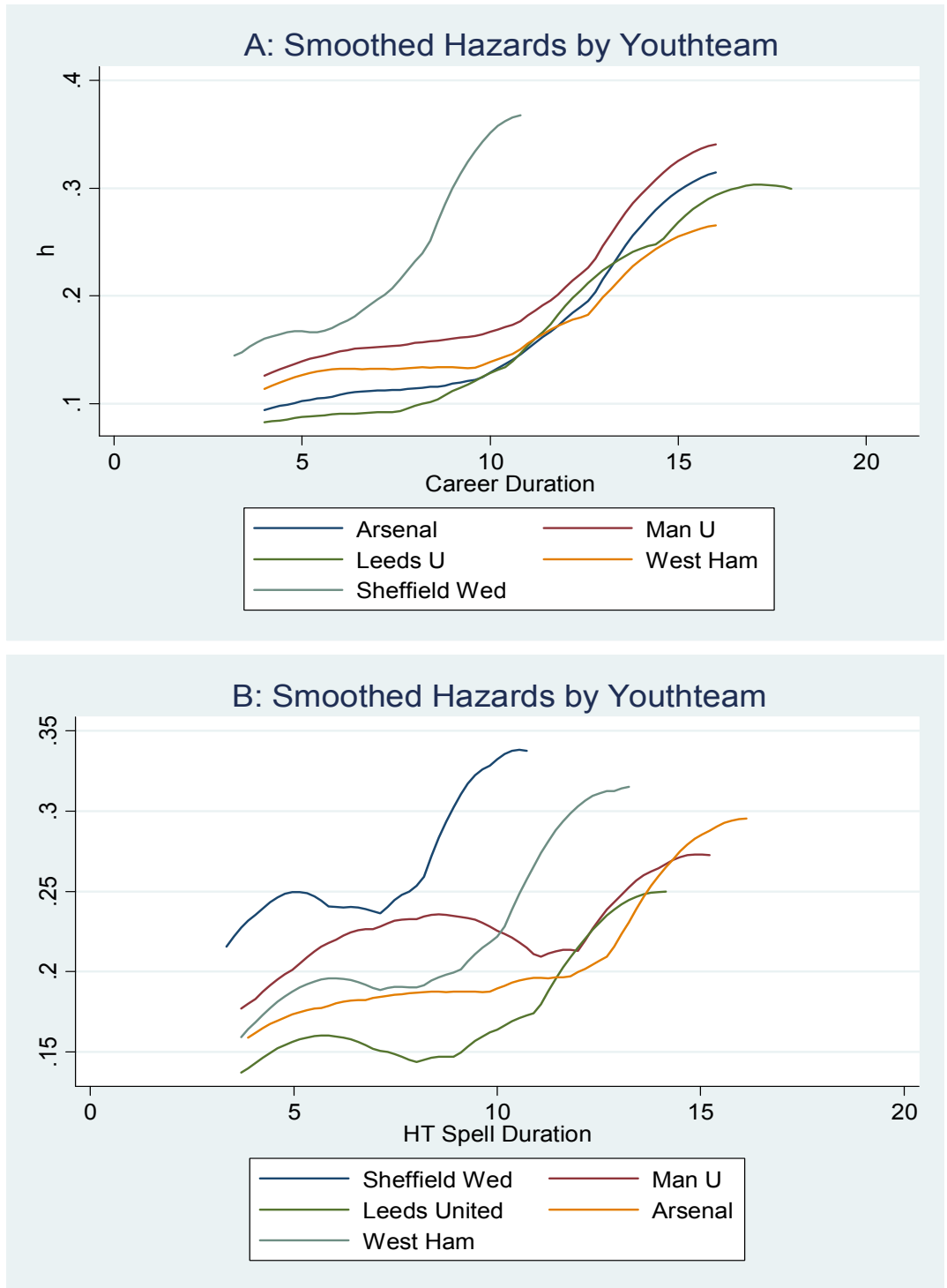

Figure 1.3: (A) Empirical Hazards by Youthteam for Career Duration, (B) Empirical Hazards by Youthteam for HT Spell Duration 

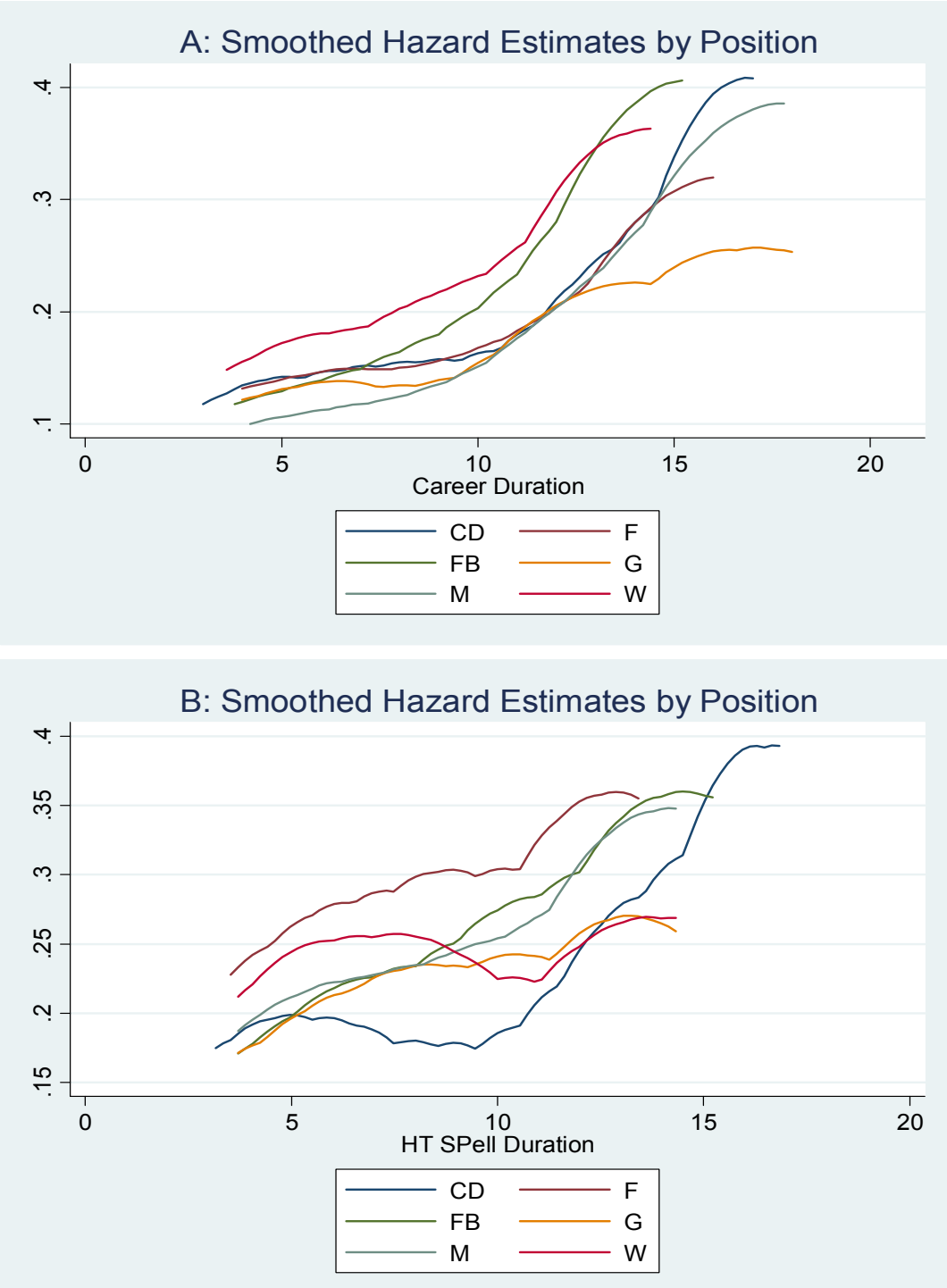

Figure 1.4: (A) Empirical Hazards by Position for Career Duration, (B) Empirical Hazards by Position for HT Spell Duration 

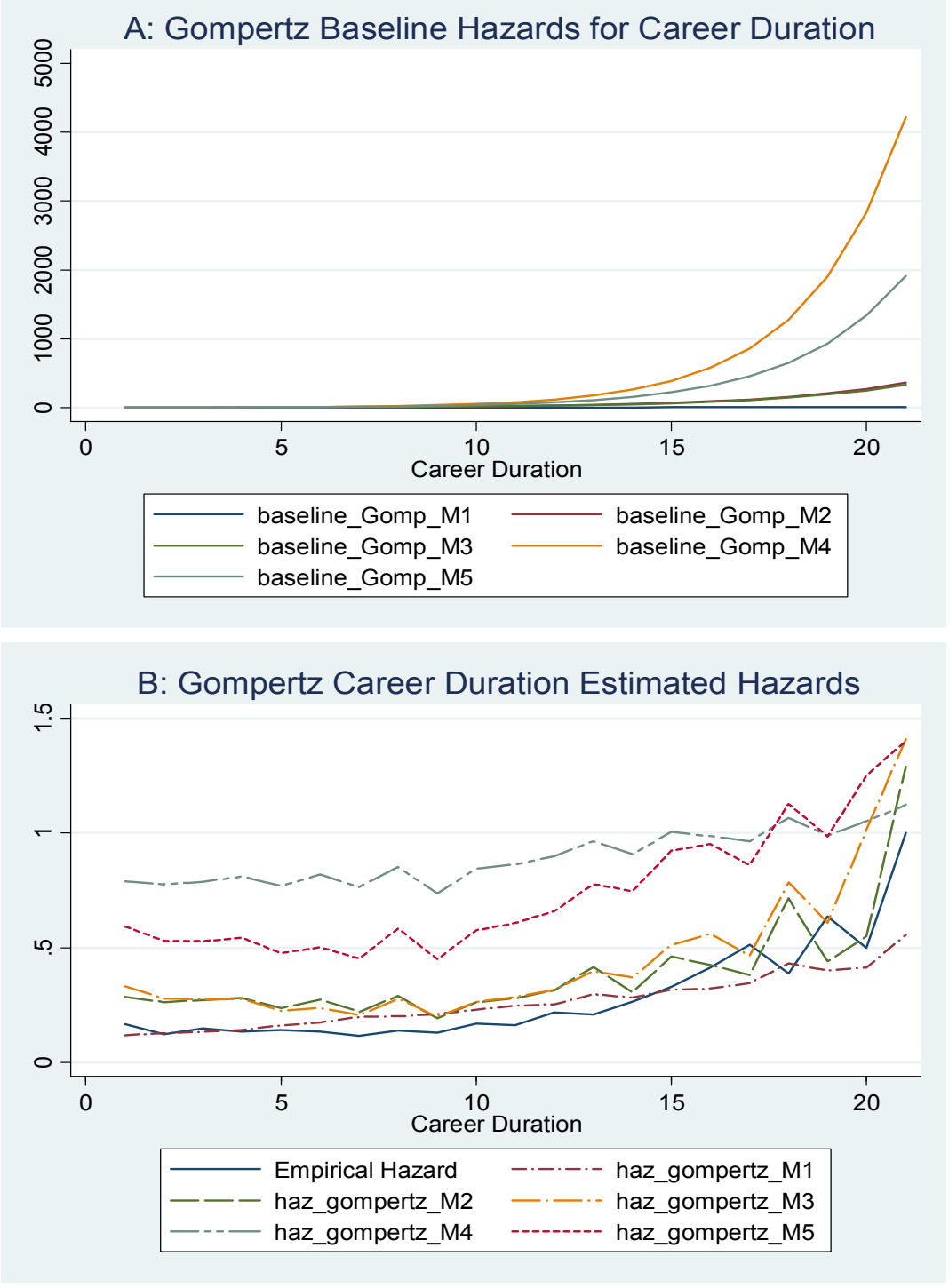

Figure 1.5: (A) Gompertz Career Duration Baseline Hazards, (B) Gompertz Career Duration Estimated Hazards 


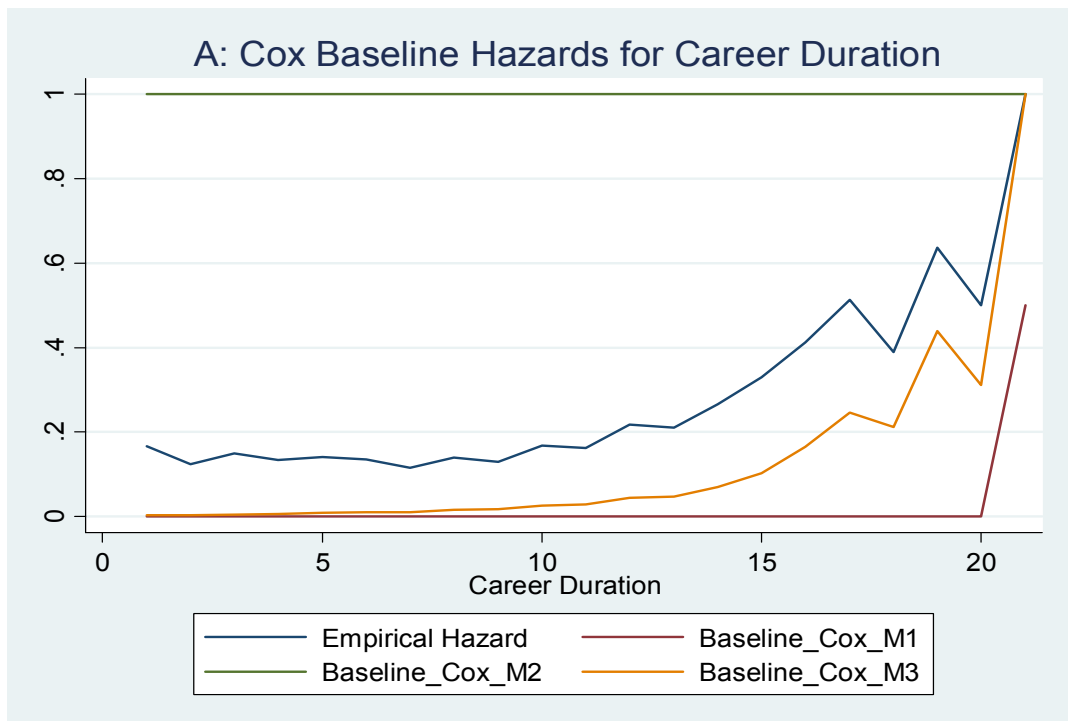

B: Cox Estimated Hazards for Career Duration

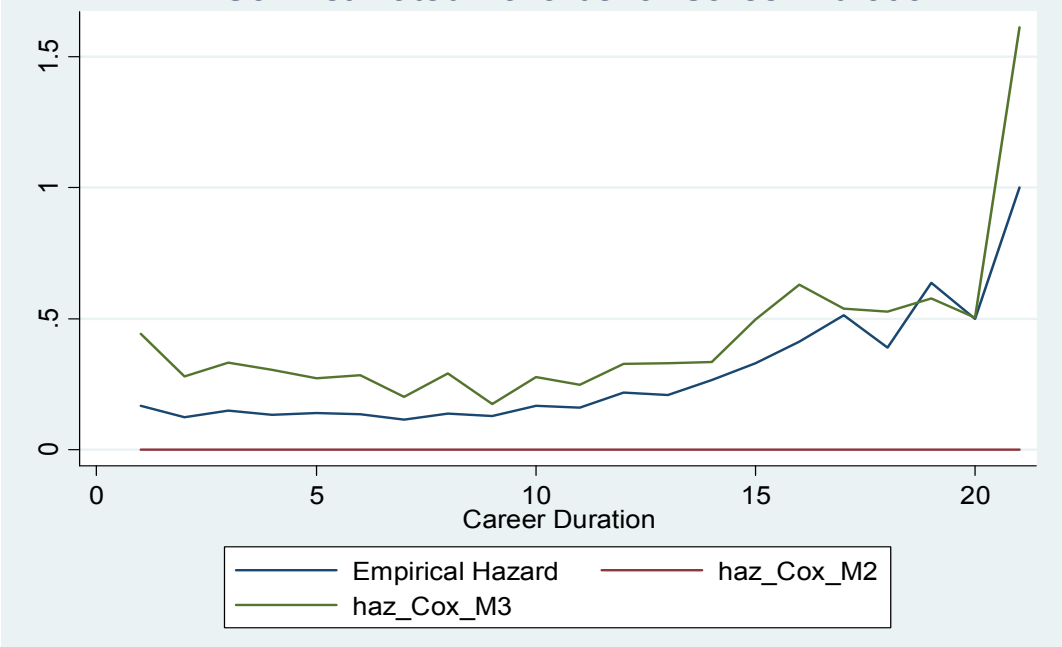

Figure 1.6: (A) Cox Semiparametric Baseline Hazards for Career Duration, (B) Cox Semiparametric Estimated Hazards for Career Duration 

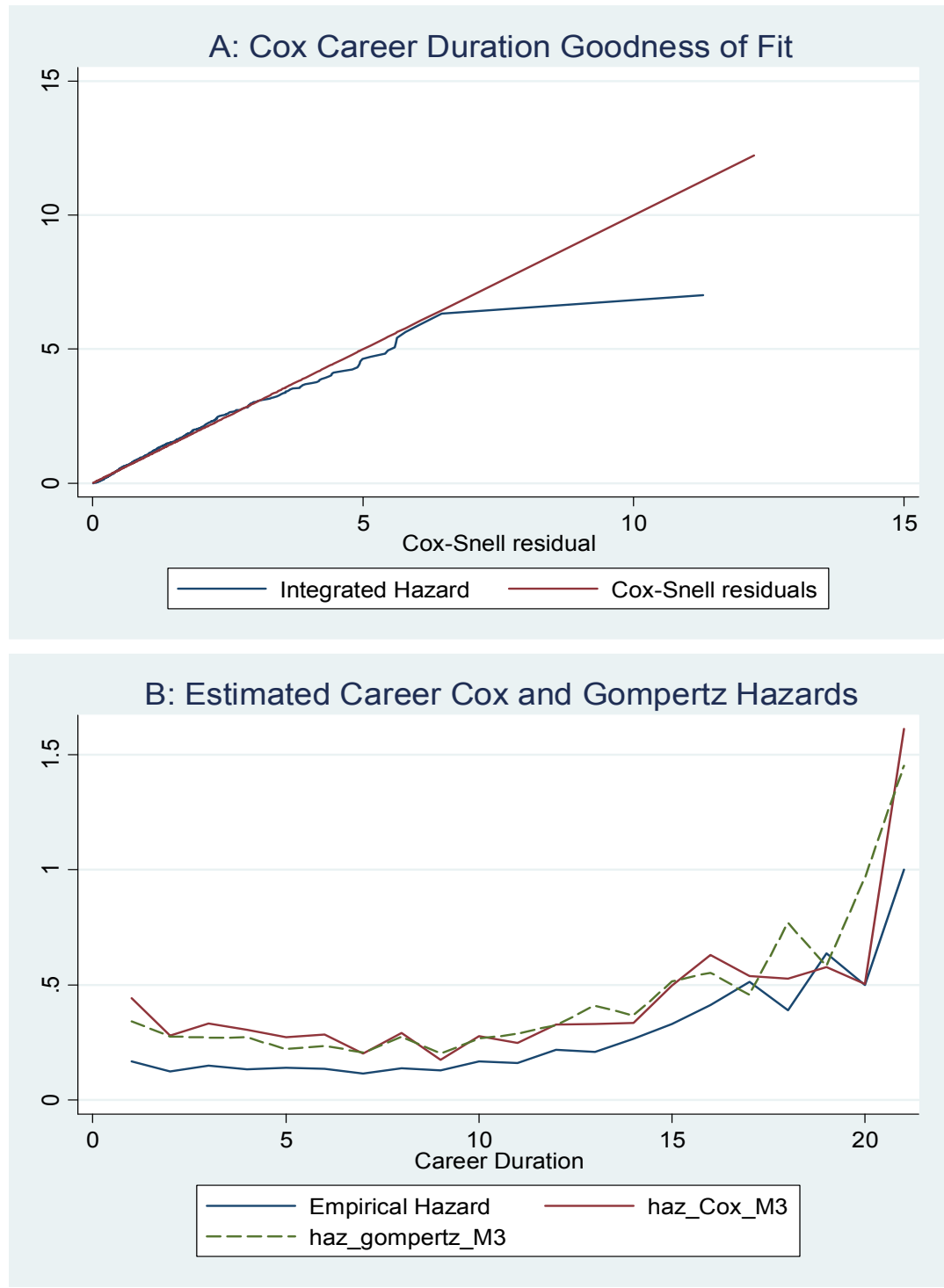

Figure 1.7: (A) Cox Career Duration Goodness of Fit Test, (B) Cox vs. Gompertz Career Duration Estimated Hazards 


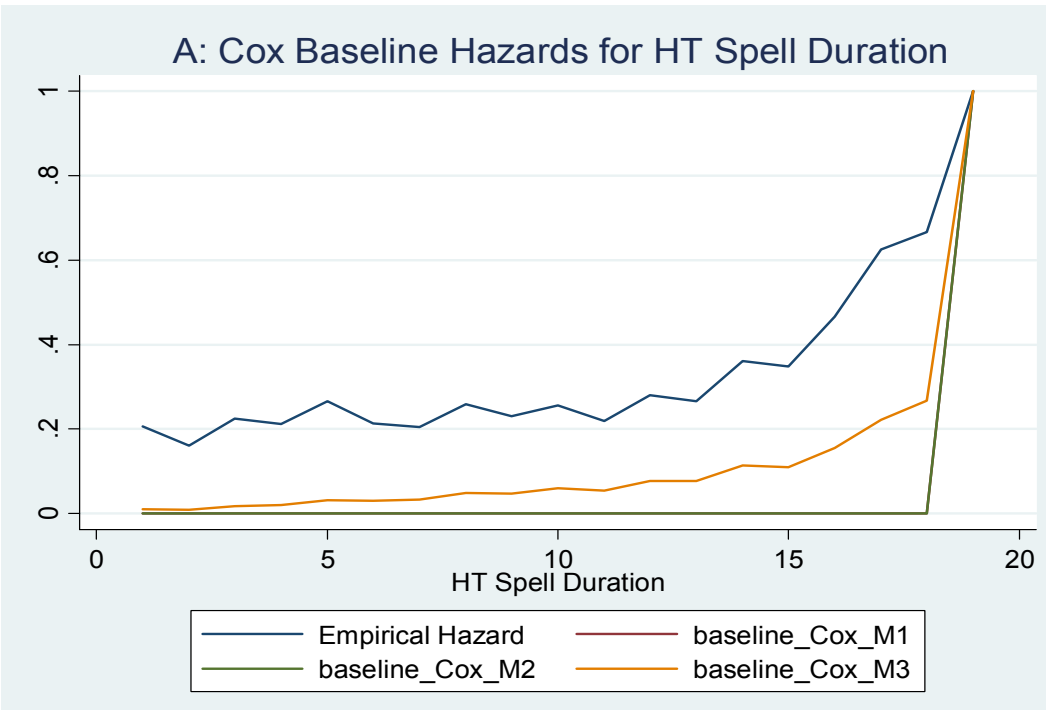

B: Cox Estimated Hazard (M3) for HT Spell Duration

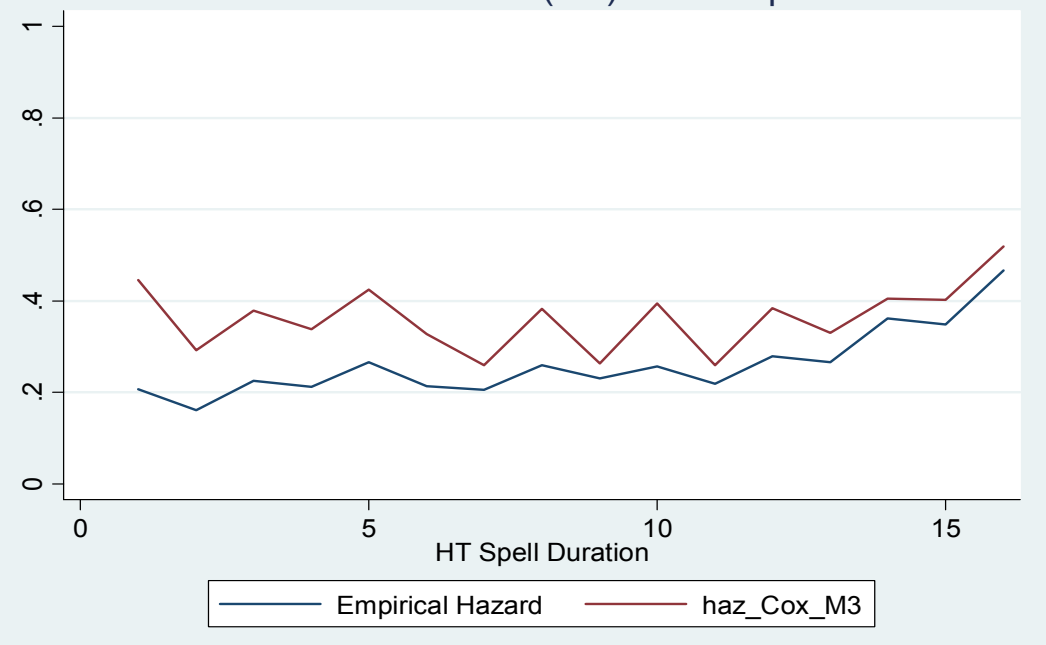

Figure 1.8: (A) Cox Semiparametric Baseline Hazards for HT Spell Duration, (B) Cox Semiparametric Estimated Hazards for HT Spell Duration 


\section{Cox HT Spell Duration Goodness of Fit}

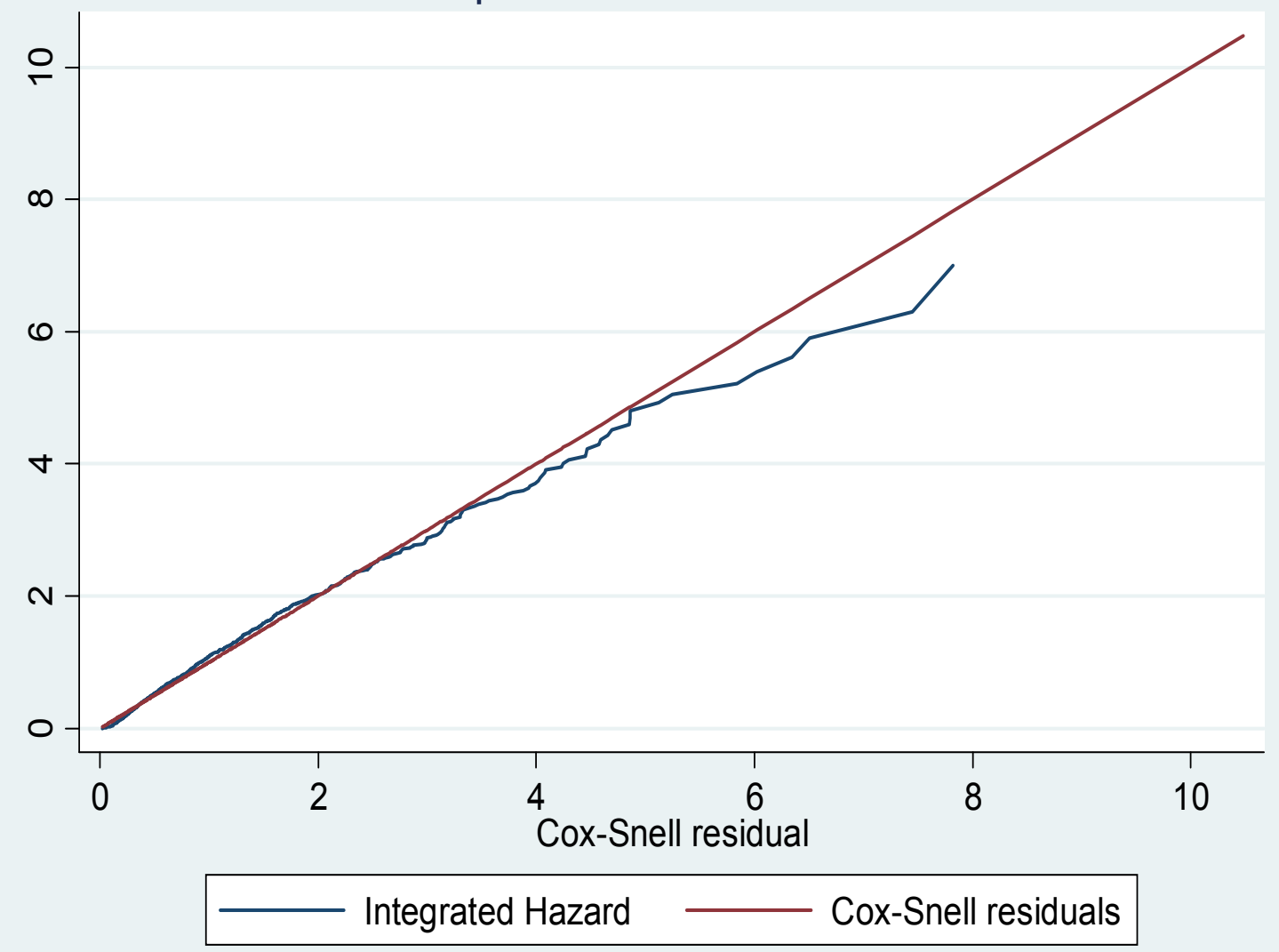

Figure 1.9: Cox HT Spell Duration Goodness of Fit Test 


\section{Chapter 2 \\ Labor market implications of institutional changes in European soccer: The Bosman ruling and its effect on productivity and career duration of players}

\subsection{Introduction}

The Bosman ${ }^{55}$ ruling in European soccer dates back to December 15, 1995. It served as the catalyst for major reform of the international transfer system in European sports, particularly in soccer. Among other changes, the ruling had a significan impact on regulations surrounding foreign player quotas. During the pre-Bosman period, clubs were restricted by the $3+2$ rule in European competitions, meaning they can fiel three foreign players and two "assimilated" foreign players per match. ${ }^{56}$ The judgement resulted in the removal of the "quota system" that was in place; European clubs were allowed to fiel as many foreign nationals from EU countries as they choose..$^{57}$ A distinction can be made between the two periods surrounding the

55 Jean-Marc Bosman was a player for RFC Liège in the Belgian First Division in Belgium whose contract had expired in 1990. He wanted to change teams and move to Dunkerque, a French team. However, Dunkerque refused to meet his Belgian club's transfer fee demand, so Liège refused to let him go. In the meantime, Bosman's wages were reduced as he was no longer a first-tea player. He took his case to the European Court of Justice in Luxembourg and sued for restraint of trade citing FIFA's rules regarding football, specificall Article 17.

56 Clubs were allowed to sign more than fve foreign players, but faced these restrictions for each matchday. Similar restrictions were in place for domestic competitions as well

57 Restrictions remained for the number of foreign players originating from countries outside of the 
Bosman ruling, transitioning from a restrictive to a more open competitive environment in terms of player movement, akin to a move from autarky to free-trade. The post-Bosman period is characterized by a shift in power from clubs to players, which is reflecte in the growth of player salaries in absolute terms and as a percentage of club revenues. Many European soccer experts/analysts have argued that these changes resulted in more uneven competitions across Europe. English press often argue that the local youth development in the country has taken a step back to foreign talent, which is also reflecte in the poor national team results on the international stage. This chapter examines the impact of the Bosman ruling from a player's perspective, and focuses on the positive aspects of a more competitive environment that lead to significan growth in stature of major European soccer leagues. These leagues now attract the best talent from around the world and their popularity is becoming increasingly global. From an economics perspective, free entry has created a more productive and efficien environment exemplifie by increasing economic rent accruing to both players and clubs; not to mention the increase in appeal to the main driver of this industry, the sport fan or end consumer that indulges in the increased quality of this consumption good.

The post-Bosman period led to a greater presence of foreigners in all European leagues, which increased competition for places and the general talent level available

EU. However, in reality clubs would get around these rules as foreign players would get citizenship in a EU country that has more favourable immigration rules, which then allowed them to be considered as domestic players in any other EU leagues. For example, many South Americans easily attained Italian, Spanish, Portugese citizenships. 
for clubs to draw from, at least for higher quality leagues where clubs are not looking only at lower-cost alternatives to domestic journeymen. As a result of increased popularity, revenues from broadcasting rights increased significantl . Transfer values and salaries trended upwards as well, and one can argue that competition for spots in top European leagues intensifie as a result. Whether or not these changes link directly to the Bosman ruling, the magnitude of the changes since the ruling cannot be overlooked. For example, aggregate revenue for all English Premier League clubs has grown from $£ 200$ Million in $1992 / 1993$ to $£ 1.3$ Billion in $2003 / 2004$. Over the same period, wage costs have increased at a faster pace from $£ 100$ Million (48\% of total club revenue) to over $£ 800$ Million ( $61 \%$ of total club turnover). The increase in turnover has been universal across the major European competitions, while the wages/turnover ratio has increased as well in all competitions (particularly in Italy, moving from $57 \%$ in $1995 / 1996$ to $90 \%$ in $2001 / 2002$ ), with the exception of the German Bundesliga where this ratio remained relatively stable around 50\%. The growth in wages/revenues ratios became more apparent from 1995/1996 and on, especially for the English Premier League and Italian Serie A. The English Premier League distanced itself from other European competitions from 1995/1996 to 2001/2002 in terms of revenue growth at an average rate of $22 \%$, from $€ 534$ Million to $€ 1.75$ Billion. The revenue from broadcasting rights increased significantl as well. The num- 
ber of matches broadcasted in England increased from 60 per season from 1992-1997 to 138 when the new deal signed with Sky Sports in $1997 . .^{58}$

Examining whether the Bosman ruling increased the average productivity and/or quality of new market entrants, that would justify the increased economic rents to a certain degree, presents an interesting avenue for analytical exploration. If this wasn't the case, one would have to attribute the increase in rents to other economic phenomena like changes in consumer preferences. The impact of intensifie competition for places can be examined through the use of economic theory. Positive spillover proponents would argue that the increased presence of more talented foreigners in other leagues has positive effects on domestic players, and will lead to a more competitive and productive environment. Competitions, in which rents are allocated as a function of the contestants efforts and ability in trying to win these rents, are a common economic phenomenon. Theoretical aspects of contests are generalizable and applicable to a wide range of activities. Examples of past studies examining strategic aspects of contests come from marketing, litigation, beauty contests, electoral competitions in politics, education filters R\&D contests, military conflict sports, etc. The competitive nature of sports provides a natural environment for the application of contest theory and testing of its predictions. Frank and Cook (1995) argued that competition for limited number of positions in certain labour markets, including a spot on a $\mathrm{Na}$ tional Basketball Association team roster, can lead to costly rent-seeking activities

\footnotetext{
58 All of the data above come from Deloitte \& Touche, Annual Reviews of Football Finance.
} 
of competing players. This chapter surveys some of the contest theory literature that may be relevant and applicable to the research question at hand. All of the relevant literature indicates that the intensifie competition brought upon by the Bosman ruling will result in higher effort and/or talent level of subsequent market entrants, which should translate into higher productivity of those players. Increased productivity has a positive effect on the players probability of winning the contest and extending their presence in the most competitive European soccer leagues, so we expect to see an upward trend in survival for players entering the market subsequent to the ruling.

One of the main contributions of this chapter is the uniquely created data set used to test the main theoretical predictions. Data is gathered for all players entering the English Premier League from $1992 / 1993^{59}$ to the $1999 / 2000$ season. Their careers are carefully tracked for each season spent in a major European competition, and all of the relevant statistics are collected for each player. Nonparametric techniques are applied to test for treatment effects imposed by the Bosman ruling; Regression Discontinuity (RD) Designs are used to estimate the Bosman policy effects on player productivity measures. The Bosman ruling provides a natural cut-off point, not influence or controlled by economic agents, that paves the way for the application of $\mathrm{RD}$ design. The results show a clear and sharp discontinuity in player productivity at the cut-off point. It is evident that post-Bosman market entrants exhibit increased productivity in soccer terms, outlined by a significan jump in the proxy productivity

\footnotetext{
59 This coincides with the establishment of the Premier League in England. The newly established
} league had 22 teams in its inagural season, after which the number of teams was reduced to 20. 
parameters. The results of the RD design are consistent with the positive spillover theoretical predictions regarding a movement to a more competitive and productive player market.

In contest theory, the increased productivity of post-Bosman entrants will have a positive effect on their chances of earning and retaining a spot on a roster in one of Europe's top soccer divisions. This notion is tested empirically through the application of survival analysis, specificall employing the Cox semiparametric model. The analysis is performed separately for players entering the market on either side of the Bosman ruling, and the results are in line with the theoretical predictions that postBosman entrants are more productive and have a higher probability of "surviving" in one of European's elite competitions. Several robustness tests, including stochastic dominance tests and Cliff's delta analysis, are performed to validate the finding of the empirical analysis and compare the hazard rate distributions of the two groups.

Section 2 discusses the theoretical motivation and its implications. Section 3 outlines the contents of the data set and presents some summary statistics of the data. Section 4 presents the results from nonparametric analyses, particularly focusing on regression discontinuity design. Section 5 provides the results of the empirical analysis for both groups of players separately, and against each other using various econometric techniques for robustness purposes. Section 6 concludes the chapter. 


\subsection{Motivating Theories and Review of Literature}

Globalization and opening up of labour markets has created many challenges and debates on the impact of immigration on the home country's economy. Among relevant theories, a few stand out in terms of definin the differing characteristics of post-Bosman entrants. Positive spillover hypothesis proponents suggest that the removal of restrictive practices and policies will lead to a more competitive environment and productive interaction among incumbents and foreign entrants. Another relevant branch of economics, contest theory, suggests that the necessary talent level to enter and survive in top-level leagues would be higher for post-Bosman entrants; The increased productivity of these players could be due to unobserved characteristics like ability and/or data, which is not determinable in this study.

Positive aspects of this labour market research focus on the benefit gained by home economies from skilled-worker immigration, while negative arguments suggest that foreigners depress wages and reduce home-worker employment. Battu et al. (2003) presents the "spillover hypothesis", whereby domestic workers permanently increase their human capital as a result of interaction with skilled co-workers from abroad that bring with them a new skill set. European sports, particularly around the Bosman ruling, provide a natural testing environment for many labour market questions about skilled-labour immigration. Alvarez et al. (2011) empirically test for the existence and strength of productivity spillovers from migrant to indigenous workers in European basketball, focusing on national team performance of host countries. Mi- 
lanovic (2005) and Frick (2009) empirically address a similar question in European soccer, with mixed results on the impact of immigration on improving the competitiveness of national teams on the international stage. All of these studies test the implications of these labour market assumptions and predictions at the team level. It seems natural to question whether these post-Bosman entrants are actually highly skilled or more productive in general. Analyzing the labour market at the player level should shed light on some aspects of the spillover hypothesis, and the impact of structural changes on the competitive environment.

Most literature in sports economics focused on a team's probability of winning a contest based on the efforts undertaken by its players. The labor market characteristics of competitions in professional sports at player level have been largely unexplored in contest theory literature. Frank and Cook (1995) argue that costly rent-seeking competitions for position(s) can occur in certain labor markets, including admission to Harvard or a spot on a roster of a National Basketball Association team. These environments are competitive in a sense that only one or a few contestants win the contest, while others waste their resources. Most players do not make it to the top level of their chosen sport, and this dream can last sufficientl long that their costly efforts in the process create a large enough opportunity cost that outweighs any potential benefit that they can attain. In addition to exposing their bodies to extreme physical strain ${ }^{60}$ the foregone opportunities in other sectors of the econ-

60 Arguments have been made that there are many benefit in young kids participating in competitions and dreaming of being a professional player, such as health. However, the longer this dream becomes 
omy can be substantial because many aspiring young athletes end their educational pursuit relatively early in their life and this decision is often irreversible and costly in the long-run. In fact, only the players that make it to the very top-level leagues in their sports can arguably secure a long-term future for themselves and validate the risk taken early on in their life from an economic perspective. Furthermore, it is not enough to make it to the top-level competitions, but one must aspire to ensure a lengthy stay in that competition in order to generate the type of rents that would compensate his opportunity cost of effort. Therefore, a theory of costly rent-seeking is applicable in player-level sports competitions for a spot and survival at the top-level competitions leagues of that sport. The assumption is that there is a positive relationship between duration at the top level and rent extracted. ${ }^{61}$ Contest theory can aid in the analysis of the player-level productivity effects and characteristics in the context of the changes brought upon by the Bosman ruling in European soccer. This section will briefl cover a few relevant and influentia papers in this fiel that will guide the analysis in the remainder of the chapter.

the health hazards probably outweigh all positive aspects of these activities. In addition to great physical strain that might speed up the aging process, players in many sports take supplemental drugs (legal or not) that may have a negative long-term effect on their health. The risk of injuries also factors in.

61 Rents can include a player's salary, image rights, sponsorhip deals, etc. One can even argue that personal pride and satisfaction is an intangible factor that has an effect on a player's valuation of the prize/rent accociated with playing at the top level. 


\subsubsection{Stein (2002)}

Stein's paper considers rent-seeking contests among asymmetric players, and is based on Hillman and Riley (1989). The asymmetry of players here can be viewed in terms of differing levels of skill and effort among participants, as well as their valuations of the rent. Stein's logic could be applicable in the context of this chapter, as it relates to player competitions for a first-tea spot on a club's roster. The structure is an imperfectly discriminating Tullock contest among $\mathrm{N}$ risk-neutral players trying to win and retain a first-tea place in one of Europe's top soccer leagues. ${ }^{62}$ A player's goal is to ensure the longest possible stay (survival) in the top-level soccer leagues in Europe. Much like Berlinschi, Schokkaert and Swinnen (2013), this chapter stipulates that players differ in terms of their innate skill level $s_{i}{ }^{63}$ and effort $e_{i}$, which are largely observable to club managers, coaches and scouts. ${ }^{64} Y_{i}$ represents the value player $i$ places on the rent/prize; these values can also represent differences in utilities of winning the same rent. The players' valuations of rents and their skill levels are ordered in a decreasing manner of their product: $s_{1} Y_{1} \geq s_{2} Y_{2} \geq \ldots \geq s_{n} Y_{n}>0$, where $s_{i} \in$ $[0,1]$ for simplicity purposes. The probability that player $i$ wins the contest is given

\footnotetext{
62 Stein's paper is a one-shot all-pay contest for a single prize/rent. In reality, players in European soccer are competing for more than one position and in mutltiple top-level leagues. Therefore, its applicability is restricted by these factors but the general conclusions that outline the nature of the competition and player-level effects of changes brought upon by the Bosman ruling are useful.

63 A player's skill level is a combination of his natural talent and human capital or tactical knowledge attained.

64 This is particularly plausible in terms of ex-post evaluation of players after their performance has been observed and assessed at season end.
} 
by the contest success function (CSF):

$$
P_{i}=\frac{s_{i} e_{i}}{\sum_{j=1}^{n} s_{j} e_{j}}
$$

If all players expand the same level of effort, the probability that player $i$ wins the contest is proportional to his innate skill level, $s_{i}$, which can be interpreted as the rate at which effort is converted into effective productivity. This indicates that a player's skill/talent alone is not enough to guarantee him a spot at the most competitive level of European soccer. Rather, it is a combination of skill and effort, assessed against other participants of the contest, that is a major determinant of a player's chances of winning the contest and ensuring a prolonged presence in top European leagues. For the purposes of this discussion, let $a_{i}=s_{i} e_{i}$ defin a player's productivity, which is dependent on his talent or natural ability, as well as his effort in employing that talent. The expected payoff to player $i$ is:

$$
E_{i}(e)=P_{i} Y_{i}-e_{i}
$$

where $e=\left(e_{1}, e_{2}, \ldots, e_{n}\right)$ is a vector spanning the expenditures of all competing players. Maximizing the expected payoff with respect to $e_{i}$ (and setting the derivative equal to 0 ), allows for an elegant solution to be obtained:

$$
a_{i}=\sum a_{j}-\frac{\left(\sum a_{j}\right)^{2}}{s_{i} Y_{i}}
$$

The optimal $a_{i}$ will be ordered in the same manner as $s_{i} Y_{i}$, up to a cutoff point $k$, such that $a_{1} \geq a_{2} \geq \ldots \geq a_{k}>0$, and $a_{i}=0$ for $i>k$. This indicates that players 
can compensate for a lack of natural talent (skill) with increased effort, but only up to a certain threshold level of talent, below which any level of effort is fruitless (due to talent constraints) and their optimal choice is not to compete at all. Summing (2.3) over $i=1,2, \ldots, k$ yields total productivity of competing players:

$$
\sum_{i \leq k} a_{i}=\left(\frac{k-1}{k}\right) \widehat{Y}_{k}
$$

where $\widehat{Y}_{k}=\left[\frac{1}{k} \sum_{i \leq k} \frac{1}{s_{i} Y_{i}}\right]^{-1}$ is the harmonic mean of $s_{i} Y_{i}$ for the sequence of players that are in the competition. Finally, from (2.3) and (2.4) we can solve for a player's probability of winning the contest or extending his stay in a top European competition:

$$
P_{i}=1-\left(\frac{k-1}{k}\right) \frac{\widehat{Y}_{k}}{s_{i} Y_{i}},
$$

which can serve as an index of a player's strength relative to competing players because $P_{1}>P_{2}>\ldots>P_{k}>0$ is ordered in the same fashion as $a_{i}$ and $s_{i} Y_{i}$. A player's composite strength is determined by an interaction of his skill level and his valuation of the rent.

Hillman and Riley (1989) demonstrate that the number of participants in a contest increases as players become more homogeneous, as is the case in the postBosman period due to increased labour mobility and the general talent pool competing for the same number of first-tea spots in a given top-level European league (i.e. English Premier League). The increase in $k$ will have a positive impact on total productivity in the league, and total rent dissipation. This prediction is in line with the 
contest theory literature indicating that reductions in heterogeneity of players can be important for generating high expected effort, or productivity in this case. The removal of barriers prompted by the Bosman ruling has certainly led to an increased number of foreigners in the top European leagues. The increased pool of players competing for spots has narrowed the talent gap among competing players, as well as the differences in player valuations of the rent, leading to a reduction in player heterogeneity and a more productive environment. Gradstein (1991) and Stein (2002) show that rent dissipation is decreasing in the asymmetry between players' talent levels, which is supportable by market data (post-Bosman) indicating significan growth in revenue, player salaries, and stature of the top European competitions. The reduction in asymmetry, brought about by the policy change, eased the participation constraint for foreign players. The assumption of positive spillover effects on average talent levels created by a wider talent pool and an influ of foreign talent is in line with other labor market studies, including Alvarez, et al. (2011) analysis of this effect in European basketball. Therefore, players entering the competition for a first team place subsequent to the Bosman ruling should generally be more productive, which translates into higher probability of survival at the top-level leagues.

\subsubsection{Fu and Lu (2012)}

The Tullock contest is the most popular special case winner-take-all lottery contest, because it provides an intuitive and tractable form of winning probability as a func- 
tion of contestant efforts. However, a ratio-form CSF of this kind does not apply to multi-prize contests, like a competition for more than one first-tea spot in top European soccer leagues. Clark and Riis (1998) generalize the basic Tullock that allows for distribution of multiple prizes, and the resulting "multiple-winner nested-contest model" resembles a sequential series of conditionally independent lotteries until all prizes are given away. $\mathrm{Fu}$ and $\mathrm{Lu}$ (2012) propose a multi-prize contest model that selects prize recipients through a noisy ranking of contestants, following pioneering work from Lazear and Rosen (1981), where output contains a deterministic component (an increasing function of effort, $g_{i}\left(e_{i}\right)$ and a noise term. Contestant are ranked in descending order based on their observed output, and a complete ranking suffice where contestants are awarded a rank based on their rank. This type of structure appears applicable in the context of this chapter; players firs compete for first-tea spots on the team that hires them, but even if they can't secure a spot on that team there are options through the transfer system where they can compete for spots on other team rosters in the league based on their relative rank. ${ }^{65} \mathrm{Fu}$ and $\mathrm{Lu}$ attempt to build micro-foundations from a noisy ranking perspective, and they prove equivalence to generalized lottery contests with multiple-prizes under certain conditions.

In this structure of $I \geq 3$ contestants and $L \geq 2$ prizes, contestants are ranked based on their perceivable performance, ${ }^{66}$ and the probability of contestant $i$ winning

65 This is particularly true for average or below average players on the best teams in the league. Even though they might not be able to secure a regular first-tea spot on those teams, they often transfer to lower ranked teams (or even teams in other top-level European leagues) in the league where they have a relative advantage over at least the average players on those teams.

66 Perceived performance is modelled according to McFadden's $(1973,1974)$ discrete choice frame- 
a prize $V_{l}$ is given by the probability he is ranked at the $l^{\prime} t h$ position. The contest embraces Konrad's (2009) notion that players compete to "get ahead of others". The ex-ante likelihood that contestant $i$ achieves top rank coincides with the popular ratioform CSF (as in Stein's paper above) in winner-take-all contests and is given by:

$$
p(i \mid e)=\frac{g_{i}\left(e_{i}\right)}{\sum_{j \in \mathbf{I}} g_{j}\left(e_{j}\right)}
$$

The $L$ prizes are awarded to the $L$ contestants that contribute the highest $y_{i}^{\prime} s$ based on their ranks. In cases where some $g_{i}\left(e_{i}\right)=0$ (and hence output $y_{i}=0$ ), these players are ranked among the bottom-most in a random manner. Considering the structure here, there is no constraint that the number of contestants, $I$, has to equal the number of prizes or first-tea spots, $L$. Therefore, if we assume that $I \geq L$, only the $i$ contestants ranked below $L$ will secure a first-tea spot in the top leagues, and the rest will be eliminated from the competition. ${ }^{67}$ If we assume $g_{i}\left(e_{i}\right)=s_{i} e_{i}$ (as in Stein's paper cited above, where CSF is the basic Tullock win probability given a player's effort and ability combination) we can get similar results and theoretical predictions as in the single-prize contest mentioned above. The cut-off point in Stein's paper can be interpreted here as a natural participation and prize/spot allocation threshold that stipulates a certain level of combined effort and ability necessary

work, with a deterministic part and noisy signal $\left(y_{i}\right)$ :

$$
\log y_{i}=\log g_{i}\left(x_{i}\right)+u_{i}
$$

where $u_{i}$ reflect the imperfections in measurement of performance and evaluation process. The noisy term is assumed to be drawn from a type I extreme-value distribution.

67 Depending on the number of contestants versus the number of prizes, this could mean that there is a cut-off point for $y_{i}$, with all players producing below this level exiting the competition. 
to secure a first-tea spot or remain in a top-level European soccer competition. ${ }^{68}$ The Bosman ruling opened up the EU leagues and significantl increased the competition for first-tea spots, particularly in the highest ranked leagues that typically provide the highest rents to players. As a result, one can safely assume the average talent level and/or effort of players entering these top-ranked European leagues in the post-Bosman period increased, ${ }^{69}$ resulting in an increase in the rent allocation cut-off point. Hence, the productivity levels and inherent rankings of Post-Bosman entrants are assumed to be higher than their pre-Bosman counterparts, which should result in a comparative advantage ensuring longer survival in the top-leagues for these players.

\subsubsection{Rosen (1985)}

Lazear and Rosen (1981) and Rosen (1985) started the formal study of internal labor market tournaments and structures that reward individuals/employees (or only their subset) based on their performance. The employees exert effort in trying to win a prize. Rosen's paper (1985) studies sequential games of survival generally applicable to career games, assuming rewards are increasing in survival. A player has option

\footnotetext{
68 Stein's set-up is only used here as an example, but the applicability is not constrained to this specificatio because of its relevance to a wider range of ratio-form functions. Essentially, if we assume the number of prizes is less than the number of competing players (very realistic), then a natural cut-off point will arise regardless of which ratio-form win technology is used.

69 While the ruling did not have an impact on domestic players (from a legal perspective), it intensifie competition with more talented foreigners seeking rents abroad. For example, the appeal of salaries in the English Premier League has increased competition for places with foreigners gaining significan share of the player market since the Bosman ruling. The quality of play and average talent level in the league increased gradually as well, and one can see some of the best players in the world appearing in the English Premier League. The assumption is consistent with related work in European basketball by Alvarez, et al. (2011).
} 
value in extending his top-level career, but this option value expires at some point (perhaps due to age or talent limitations compared to other players). This multipleplayer game is also characterized by a rank-order scheme for players based on their assessment of value-added. Consider a game with $m$ type of risk-neutral players; the ability type of the $i$ and $j$ players is indexed by $I$ and $J$, respectively (both take on $m$ possible values). Let $e_{s i}$ and $e_{s j}$ represent the effort expended by the two types of players, with $s$ stages remaining in the game. Also, let $\lambda_{I}$ and $\lambda_{J}$ represent their abilities or natural talent. The probability that player of type $I$ wins against a player of type $J$ is assumed to follow the law:

$$
P_{s}(I, J)=\frac{\lambda_{I} h\left(e_{s i}\right)}{\lambda_{I} h\left(e_{s i}\right)+\lambda_{J} h\left(e_{s j}\right)}
$$

with $h(e)^{70}$ strictly increasing in $e$ and $h(0)=0$, so a player increases his probability of winning by exerting greater effort given own talent and opponent's effort and talent. Rosen assumes a Poisson proportional hazards form with $H_{i}=\lambda_{I} h\left(e_{i}\right)$ representing the probability of exit at $t,{ }^{71}$ given that player i has survived up to $t$. The unconditional duration density is given by $f_{i}(t)=H_{i} \exp \left(-H_{i} t\right)$. Larger values of efforts by players are associated with higher average quality of play. Greater effort increases the probability of surviving, but involves added costs, $c(e)$. In this type of game, the anticipated value of surviving depends on how the player assesses future effort expenditure and behavior, and current actions depend on the behavior of the

70 For simplificatio purposes, the winning technology $h(x)$ is assumed to be the same at each stage.

71 Essentially, $t$ represents the player's duration from the time he entered the top-level European soccer league. Rosen presented this in the context of a racing game to the finis line, but the hazard function can be interpreted as survival function in the context of this paper. 
current opponent and on anticipated actions of possible future opponents. Differentiating player $i$ 's best response function with respect to player $j$ 's effort yields:

$$
\frac{\partial e_{i}}{\partial e_{j}}=\frac{c_{i}^{\prime}\left(\frac{h_{j}^{\prime}}{h_{j}}\right)}{-D\left(\lambda_{I} h_{i}+\lambda_{J} h_{j}\right)}\left(\lambda_{I} h_{i}-\lambda_{J} h_{j}\right)^{72}
$$

Player $i$ 's best response is decreasing when the opponent's effort is sufficientl large. The turning point (beyond this point it does not benefi to keep pace with the opponent because it is too costly) is at $\lambda_{I} h_{i}=\lambda_{J} h_{j}$, which can occur at $e_{i}=e_{j}$ for equally talented players, at some value $e_{i}>e_{j}$ when the opponent is more talented $\left(\lambda_{J}>\lambda_{I}\right)$, and at some value $e_{i}<e_{j}$ when the opponent is weaker $\left(\lambda_{J}<\lambda_{I}\right)$. Once again this suggests that players can stay competitive by compensating for a lack of talent with additional effort, but only until a certain point. If one considers post-Bosman entrants into a top-level European soccer league to be more talented (or perceived to be more talented) or the market to be more competitive in terms of players exerting higher effort, less talented players (pre-Bosman entrants) will become less competitive and exert less effort negatively impacting their probability of survival. Rosen's results extend to n-way (instead of pairwise) comparisons at each stage, under certain conditions like symmetric win technologies and equally talented contestants. Even if players entering the market on either side of the Bosman ruling were equally talented, the increased number of contestants and intensity of competition will generate a real or perceived increase in effort of post-Bosman players that will negatively impact the effort/productivity of some pre-Bosman contestants (beyond the turning point) causing an exit from the market. 
Rosen's paper also considers games with heterogeneous contestants with known talents, and argues that the conditional distribution of survivors in sequential games exhibits a larger mean talent as the game proceeds because the value of continuation is larger for stronger players than weaker ones. The strong are contending for toplevel prizes, while the weak are contending for lower ranking prizes. If we assume that players entering the market after the Bosman ruling are stronger on average, they will compete for survival in the top European leagues with stronger players from the pre-Bosman period, while the weaker pre-Bosman players will gradually exit and compete for positions in lower-level leagues.

Rosen also considers heterogeneous contestants with unknown talents. This could be reflect ve of the situation brought upon by the Bosman ruling and the opening of EU soccer countries to a rather unknown and wide pool of foreign talent. In this scenario all players choose the same effort levels and probability of winning/surviving are based on each type's talent level, so survival probabilities are increasing for stronger players. The weak are eliminated at the largest rate when the there is an equal proportion of weak and strong players in the game. The value of survival depends on a player's assessment of own and opponents' talents at any stage, and the value of information enters the model dynamically. Equilibrium conditions indicate that the value of survival is increasing in own assessment of talent. The value of information is small when contestants are similar in terms of talent. The marginal 
effect of effort on the win probability is decreasing in the extent of heterogeneity in the player population and uncertainty in own assessment of type at each stage.

Looking at the effects of the Bosman ruling from any scenario above suggests that pre-Bosman market entrants' best response functions will be characterized (whether due to higher average talent level or increased effort of post-Bosman players) by lower effort levels and productivity, leading to a higher average hazard rate as compared to the post-Bosman entrants.

\subsubsection{Other Relevant Discussions}

Ryvkin (2009) looks at three different tournament formats (one stage contests, binary

elimination, and round-robin tournaments) or contests and considers the effects of weak heterogeneity of players on equilibrium strategies. He shows that a player's equilibrium effort can be characterized by his own ability and the average ability of all other players, and that a player's reaction to a change in his own relative ability is stronger than to a change in the relative ability of other players. In the dynamic game context, a player's equilibrium effort at stage $t$ decreases in the relative perceived expected opponents' abilities. Even though the tournament is binary in nature, the logic of the effect of the expected abilities of future opponents is relevant. Once the Bosman ruling took effect, it is plausible that the perceived ability of future market entrants revised the expectations of incumbents. The perceived threat from increased ability of future competitors possibly impacted the players in the market up to that 
point, particularly the most recent entrants who haven't established their reputation in the league yet. Ryvkin (2013) mentions that all other things being equal, players with higher ability are expected to perform better, so policies directed at increasing average ability in the competition would be beneficial ${ }^{73}$ The Bosman ruling can be viewed as such a policy, even though it was not implemented as a result of direct intent of the organizers, but was brought upon by exogenous factors. Konrad (2009) provides a terrifi review of literature focusing on the strategic aspects of contests. One source of asymmetry in these contests is represented by a player's cost-of-effort function, and changes in these functions can be unintended and brought upon by a policy change that was not motivated by the contest, which is representative of the Bosman ruling itself. There is also a possibility that the fl xible supply of talent in the post-Bosman period led to an uneven distribution of rents, with superstar players earning far greater rents than average players in the league.

\subsection{Data Set and Summary Statistics}

The empirical study is based on a uniquely collected data set consisting of all players that entered ${ }^{74}$ the English Premier League since its establishment in 1992/1993, ending with the 1999/2000 season. The focus on new entrants aids in assessing players with similar characteristics, outside of the profil changes brought upon

73 Ryvkin argues that, in sports, higher effort exerted by athletes makes competitions more attractive to spectators and results in increased revenues to teams and organizers.

74 Entering the league means making at least one firs team appearance for any team in the English Premier League during the study period. 
by the Bosman ruling. The ruling itself occurred in December of 1995 (but effectively came into practice in January of 1996) and splits the data on entrants almost evenly on either side in terms of the seasons considered here. The career of each player that entered the English Premier League during this period is tracked and all publicly available and relevant statistics are captured for each season spent in a top European league. ${ }^{75}$ The data were collected using numerous internet sources, with the following sites serving as the backbone for the data generating process: http://www.worldfootball.net/, http://www.transfermarkt.co.uk/en, http://www.soccerbase.com/. The information gathered includes: player's name, season of entrance ("year of entry") and each subsequent season spent in a top European league, player's source (whether he was brought up through the youth ranks, transferred in from a lowerlevel domestic club, or transferred in from a foreign club), the associated cost of acquiring his rights ${ }^{76}$ ("player cost"), each top-level club that a player appeared for in his career and relevant transfer fees paid for his rights, the ranking ("TeamPos") for each team that a player appeared for in each season, the share of foreign players in the top-level league for each relevant season ("forshare"), player's position, age at entry ("age of entry") and each subsequent season, nationality (UK, EU, and nonEU), appearances ("apps"), minutes played ("mpg"), goals scored, own-goals, yellow and red cards, international appearances ("intapps") and minutes played ("intmpg"),

75 Top European leagues consist of: English Premier League, Spanish La Liga, German Bundesliga, Italian Serie A, and the French Ligue 1.

76 For example, players brought up through the respective club's youth system are assigned a zero transfer value since the club does not have to bid for their rights in the transfer market. 
the player's transfer status for each season (whether the player stayed with the same club or transferred to another club for free or for a fee), and finall whether and for what reason ${ }^{77}$ a player exited the top-leagues in each season (most frequent reason for exiting was moving to a lower division team, whether it was by way of transfer or relegation).

Table 2.1 breaks down player entries by the Bosman ruling and player source. A total of 861 player entries are observed during the study period, of which 504 entered during the post-Bosman period and 357 entered during the pre-Bosman period. One statistic that particularly stands out is the number of foreign entrants in the postBosman period compared to the pre-Bosman period, which is more than three times higher (242 compared to 79). An increase was to be expected as the Bosman ruling removed restrictions surrounding player movement within the EU countries, but the magnitude of the increase in the firs four years after the ruling certainly stands out. We observe that nearly half of all entrants are foreign sourced in the post-Bosman period compared to $25 \%$ in the pre-Bosman period, which is predicted by the theoretical model as a result of easing the participation constraint for these players. The Bosman ruling did not impact the number of youth entrants in totality, but in relative terms as a percentage of total entrants it did have a negative effect of more than $10 \%$. Players sourced domestically suffered the most as the number of entrants decreased in total and relative numbers. The Bosman ruling removed certain trade barriers and

\footnotetext{
77 Players exiting the market due to injury are excluded from the fina sample to improve the relia-
} bility of the statistical inference. 
significantl increased the talent pool that EU based clubs can draw from. Therefore, it is not surprising that there was a shift toward foreign players and that the number of entrants significantl increased in the post-Bosman period as the talent pool available to clubs grew in size and scope. The theoretical predictions are consistent with these summary statistics and market information mentioned earlier.

Table 2.2 provides some basic summary statistics for certain variables in the data set, in total and broken down by pre and post-Bosman periods. Average appearances were higher by one for the post-Bosman entrants and average international appearances were higher by 0.5 as well for the same group. The average transfer cost for players entering on either side of the Bosman ruling was not materially different, and the highest observed transfer cost was around 40 million British pounds. The youngest player to enter the market during the study period was 16 and the oldest player still active at the top-level was 41 . The average age of post-Bosman entrants was higher by just over one year compared to pre-Bosman entrants. The share of foreign players significantl increased in the post-Bosman period, approximately by $17 \%$ at the mean level. This concept is further approached through a more technical empirical approach that tests the effect of the Bosman ruling on the competitive structure of English and European soccer. 


\subsection{Nonparametric Methods}

Estimating the existence and size of a discontinuous jump can be accomplished by comparing means in small bins of $Z$ to the left and right of $Z_{0}$ or with a regression of various powers of $Z$, an indicator $B$ for $Z>Z_{0}$, and interactions of all $Z$ terms with $B$. A very basic method calculates the mean of each variable of interest for each bin on the time line selected, almost like a histogram. This rather simplistic visual approach paves the way for a more formal analysis below. Figures 2.1 and 2.2 present the means for two productivity proxies (appearances and minutes per game, respectively) for each of the four bins ${ }^{78}$ selected on either side of the Bosman ruling, with a quadratic fi line connecting the given bin means. The graphs suggest there is evidence of a jump in the conditional means for these two covariates around the Bosman ruling, particularly for the sub-sample of players that is nearest to the cut-off on both sides. Other productivity proxies, like international appearances, also display a discontinuity at the cut-off, while other variables examined (i.e. transfer costs) do not show signs of discontinuities.

\subsubsection{Regression Discontinuity (RD) Design}

The relevant theory suggests that there is a discontinuity in player productivity variables as a result of policy changes for players on either side of the cut-off point. The Bosman ruling provides a natural setting for testing the treatment effect and variable

78 The results are consisstent for other bins tested as well, but only the graphs for four bins are presented. 
discontinuities around that specifi cut-off point, which is define by the date the ruling came into effect. Players entering the market subsequent to the Bosman ruling were subject to a different competitive and regulatory environment than players that entered the market ahead of the ruling. All relevant Labour Economics theories mentioned suggest that post-Bosman players should possess different characteristics, perhaps driven by higher ability and/or effort. As such, the post-Bosman entrants are viewed as the treatment group; The institutional changes arising from the ruling are viewed as treatment effects to subjected post-ruling entrants.

The RD approach is appealing because it provides the greatest internal validity among all quasi-experimental methods that estimate a local average treatment effect (LATE) at the cut-off point. In addition, a major advantage of the RD design against competing methods is its transparency using graphical methods. A proper RD design is characterized by a set of assumptions that are relevant in the context of this analysis:

- Two groups: Players entering before and after the institutional change represented by a dummy A.

- Two periods of time around the cut-off point represented by a dummy variable T.

- Players cannot precisely manipulate the assignment variable to influenc whether they receive the treatment or not. 
- Treatment indicator define by the dummy $B=A x T$.

- Forcing and control variables that are continuous around the cut-off point.

The identificatio of the policy impact on player productivity can be estimated by the following reduced form specification

$$
Y_{i}=\alpha+\beta B_{i}+f(X)+\varepsilon_{i}
$$

where $B=0$ for players entering the top tier of English soccer prior to the Bosman ruling, and $B=1$ for players entering subsequent to the ruling. The assumption of continuity of the baseline covariate effect of a player's transfer value around the cut-off is estimated by $f(X)$. Optimal bandwidth is obtained a la Imbens and Kalyanaraman (2009) method that minimizes the squared bias plus variance. The estimation is performed at 4 bandwidth levels around the cut-off, specific lly at year 1, year 2, year 3, and year 4 on either side of the Bosman ruling. The different bandwidths serve as robustness checks in the estimation of the potential jump in the treatment outcomes around the Bosman ruling. Other control variables (i.e. the relative league strength of the team employing the player) are used to test if the Bosman effect is appropriately estimated. The treatment effect is identifie as:

$$
\beta=\frac{\lim _{\varepsilon \downarrow 0} E[Y \mid B=\varepsilon]-\lim _{\varepsilon \uparrow 0}[Y \mid B=\varepsilon]}{\lim _{\varepsilon \downarrow 0} p(Z)-\lim _{\varepsilon \uparrow 0} p(Z)}
$$

If the impact of treatment is immediate we have a sharp RD design (discrete jump at cut-off) and denominator becomes 1 . If the effect is fuzzy around the cut-off, 
the denominator is in the $[0,1]$ interval. The treatment parameter is estimated using local polynomial regressions on either side of the cut-off point:

$$
\min _{\beta} E\left[Y_{i}-\alpha-\beta\left(Z_{i}\right)\right]^{2} K\left(\frac{Z_{i}-Z_{0}}{h}\right)
$$

where $K\left(\frac{Z_{i}-Z_{0}}{h}\right)$ is a triangle Kernel function that gives more weight to the observations closer to the cut-off, and $h$ is the bandwidth level.

The results are presented in Table 2.3. It appears the Bosman ruling generated positive productivity treatment effects as predicted by the theoretical model. Players entering the English Premier league in the post-Bosman period are playing approximately 6-7 more minutes per game and making 2-3 more appearances per season than the pre-Bosman control group. ${ }^{79}$ All of the coefficient are statistically signifi cant at the $5 \%$ level, with all but one significan at the $1 \%$ level. Variability is reduced for larger windows as expected, without visible losses to precision. Controlling for other covariates, like player age at entry and his respective team's ranking in the league, does not impact the results for the tested outcomes. In addition, the continuity assumption is satisfie for players' transfer costs around the cut-off, as there is no evidence of a jump or discontinuity at that point. The table also presents results for international appearances and minutes per game, which display statistically signifi cant results for 1 and 2 year windows at the $1 \%$ level, whereas the results for 3 and 4 year windows are insignifican at any reasonable level. This is not that surprising considering only a relatively small portion of players examined made international

79 The optimal bandwidth for mpg and apps was 2.12 and 2.46, respectively. 
appearances during the study period. Nonetheless, the significan results for 1 and 2 year windows imply positive treatment effects in terms of player productivity even at the international level.

A graphical illustration of the results for minutes per game and appearances is presented in Figures 2.3 and 2.4, respectively. The treatment effect (jump) at the cut-off is evident in all figures and this result is robust to different bandwidth selections around the Bosman ruling. Therefore, the RD design results lend support to the theory predicting higher productivity of players entering the market in the postBosman period versus players entering in the pre-Bosman period. The reduction (or removal in some cases) of barriers to free movement of labor increased the average talent and/or effort levels of new entrants in the English Premier league, resulting in higher productivity for this group. This should in turn lead to an increased probability of survival in top European leagues for the post-Bosman treatment group. This notion will be subject to further empirical analysis in the next section.

\subsubsection{Nonparametric Survival Analysis}

The RD design established that there exists a sharp discontinuity in variables measuring player productivity around the Bosman ruling. More formal models for survival analysis are presented subsequently to test for differences in duration patterns among the two groups examined. Nonetheless, some figure based on nonparametric tools are useful in the analysis process. The empirical survivor function for the 
pre-Bosman entrants is a little steeper, especially in the firs few years, than for the post-Bosman entrants. This might indicate that there are more variations in the talent level of pre-Bosman players, which results in faster exits for the below average players. This intuition is further supported by Figure $2.5,{ }^{80}$ which presents the empirical hazard functions for the two groups of players. The empirical hazard is higher for pre-Bosman entrants versus post-Bosman entrants indicating a higher exit probability for the same group at average player level. The upward sloping hazard curves indicate a positive duration dependence, which is not surprising considering the age driven physical limitations encountered by professional athletes in most competitive sports. In addition to visual examination, nonparametric tests (Log-rank and Wilcoxon) were performed and the results suggest there are sub-group differences in the survivor functions of pre and post-Bosman entrants.

\subsection{Empirical Analysis}

The nonparametric analysis lends support to the theory presented in this chapter, establishing the presence of a sharp discontinuity in player productivity and a difference in empirical survival measures for the two groups of market entrants around the Bosman ruling. The theoretical predictions related to their differences in the probability of winning and retaining a spot in a top-level European league are tested using

80 The negative slope at larger durations is due to outliers in the data with extremely long spell and career duration. 
survival analysis. The duration patterns of the sampled players are estimated using reduced-form hazard models that measure the instantaneous probability of exit at time $t$ conditional on having survived until that time:

$$
h(t)=\lim _{\triangle t \rightarrow 0} \frac{\operatorname{Pr}(t \leq T<t+\triangle t \mid T \geq t)}{\triangle t}
$$

Hazard models are conditioned on individual specifi covariates, including the player productivity measures (mpg, apps, intapps) ${ }^{81}$ dummies for player position and nationality, and relative measures of the strength of team employing the player. This chapter estimates the hazard rate using the $\operatorname{Cox}^{82}$ semiparametric model:

$$
h_{i}\left(t_{i} \mid x_{i}, v_{i}\right)=\phi\left(x_{i}\right) \lambda\left(t_{i}\right)
$$

where $\phi\left(x_{i}\right)=\exp \left(x_{i} \beta\right)$ is a function of the observable time-invariant covariates, and $\lambda\left(t_{i}\right)$ is the nonparametric baseline hazard for individual player $i$. The main advantage of the Cox model is that it makes no assumptions about the distribution of survival times and is robust to mis-specificat on of the baseline hazard, unlike the alternative parametric duration models. The general nature of the model allows the estimation of slope parameters for the covariates irrespective of what the baseline hazard looks like. The model assumes that the covariates will have a proportional and

\footnotetext{
81 Player productivity is difficul to measure uniformly across the different positions in European soccer. For, example goals scored can be a useful measure for forwards, but is almost useless for other positions. However, minutes played and seasonal appearances provide a strong indication of a manager's assessment of player productivity and contribution to the team.

82 The Cox PH model is also representative of Rosen's (1985) Poisson hazard rate specification
} 
constant effect that is invariant to time, and this proportional hazards $(\mathrm{PH})$ notion is tested by conducting residual-based tests using Schoenfeld residuals. ${ }^{83}$

In order to improve robustness and model specification testing for nonlinear functional form of the covariates is performed using restricted cubic spline functions a la Royston and Sauerbrei (2007) ${ }^{84}$ The linear and quadratic functional forms are both special cases of the spline fit which is capable of modelling more complex curve shapes than competing methods. As it turns out, the only covariate that displays a nonlinear fi is TeamPos, which measures the player's relative club strength in the league at each point in time.

It is also very important to distinguish between two sources of duration dependence, true state dependence (TSD) and unobserved heterogeneity or spurious state dependence (SSD). Under SSD, ${ }^{85}$ the amount of time spent by players in a certain state does not affect future probabilities of exiting or remaining in that state. On the other hand, TSD implies past experience plays a role in the probability of surviving in a given state. It's safe to assume that negative TSD will outweigh the positive TSD in the context of this chapter, given physical/age limitations of players (versus the positive effects of experience) will increase their exit probability the longer they are in a given state. The presence of SSD will result in mis-specificatio in models that

83 The Therneau-Grambsch test (1994) applied is based on scaled Schoenfeld residuals and is applicable both globally and at specifi covariate level.

84 For a detailed description of the methodology, see Radoman and Voia (2015, forthcoming). In addition, Keele (2010) provides a basic analysis of the method's advantages and improvements in model specification as well as its lack of applicability in academic literature.

85 SSD implies that observations are conditionally different (heterogenous) in terms of their hazard because individual specifi unobserved characteristics. 
do not account for it, and the $\mathrm{PH}$ property in relevant models (particularly parametric ones) would be lost. This chapter tests for individual-level unobserved heterogeneity via random effects (frailty) models that introduce SSD multiplicatively. ${ }^{86}$ The results of the frailty models indicate that unobserved heterogeneity is insignifican at any reasonable level, which allows for a more substantiative interpretation of the model results based on TSD as the main source of duration dependence.

Considering the $\mathrm{PH}$ nature of the Cox model, the results are presented as hazard ratios rather than individual covariate coefficients Hazard ratios simplify the interpretation of the results. Suppose the value of a certain covariate, $X_{k}$, is changed by an amount $\delta$. The hazard ratio is given by:

$$
\frac{h\left(\bar{t}, X_{k}+\delta\right)}{h\left(\bar{t}, X_{k}\right)}=\exp \left[\left(X_{k}-\left(X_{k}+\delta\right) \hat{\beta_{k}}=\exp \left(\hat{\beta}_{k} \delta\right)\right.\right.
$$

and if $\delta=1$, the above equation reduces to:

$$
\frac{h\left(\bar{t}, X_{k}+\delta\right)}{h\left(\bar{t}, X_{k}\right)}=\exp \left(\hat{\beta}_{k}\right)
$$

Altogether, there are four different model specification (for both groups of players) considered in this chapter:

- M1: Model with productivity parameters only

- M2: M1 plus individual specifi characteristics

86 Unobserved heterogeneity is modeled by the Gamma and the Inverse Gaussian distributions. 
- M3: M2 plus a player's relative team strength indicator

- M4: M2 plus nonlinear functional form for a player's relative team strength indicator

\subsubsection{Results for Pre-Bosman entrants}

The results of all four models estimated are presented in table 2.4. The productivity measures are statistically significan at least at the $5 \%$ level in all specifications with the exception of international appearances in M3 (significan at the $10 \%$ level). As predicted, all three covariates have a negative effect on the hazard rate, meaning an increase in productivity will reduce the players probability of exit from the top European leagues. Looking at M4, playing one additional game per season reduces the exit probability by around $4.2 \%$, while playing one more minute per game reduces the exit probability by $0.45 \%$, and making one international appearance reduces the exit probability by around $7.9 \%$. It is noticeable that as we move from M1 specificatio and control for player characteristics and team strength covariates in the other specifications the impact of first-tea appearances increases (negatively as to reduce the probability of exit) in magnitude (by almost $1 \%$ from M1 to M4) and the impact of international appearances decreases in magnitude (by 5-6\% from M1 to M3\&M4), while the effect of minutes per game increases in magnitude as well (by 16 basis points from M1 to M4). Improving model specificatio has the largest effect 
on the coefficien for international appearances, whose magnitude seems to be overstated in M1 that doesn't control for any non-productivity related covariates. The negative effect of minutes per game increases by around $35 \%$ as well in M4, meaning that playing an additional 10 minutes per game reduces the exit probability of players by around $6 \%$. This implies, as one would predict, that regular first-tea players have a higher probability of survival than brink players that make most of their appearances as substitutes. Therefore, most observed exits in the data should be by players that fail to solidify their first-tea spot on a top-level team's roster, i.e. their level of productivity (they could lack sufficien natural talent and/or effort) in matches and/or training is not satisfactory to the manager and the coaching staff. Linking this to the theoretical predictions, these would be the players that do not satisfy the competition threshold level, and are forced to exit the competition due to insufficien productivity at the top-level of European soccer.

A player's age at entry has a strong and statistically significan (at the $1 \%$ level) negative effect on the hazard rate in all specifications Interpretation suggests that each year added to a player's age results in a $4.3 \%$ increase in exit probability in M4. This result is to be expected because of physical limitations imposed by aging in professional sports, and is consistent with negative TSD that is assumed to dominate positive TSD due to experience gained. Controlling for age is very important within the data set collected considering most youth players enter the market before they turn 19, as opposed to players sourced from other teams or leagues (domestic or 
foreign) whose age at entry can vary significantl and is generally higher than for players sourced directly from youth academies. Position played and nationality of a player do not have a statistically significan effect at any reasonable levels considered. The insignifican result for position dummies is somewhat surprising considering past studies $^{87}$ on this issue and differing physical demands and public scrutiny ${ }^{88}$ faced by players in certain positions. Team position has a strong and statistically significan (at the $1 \%$ level) effect on exit probability in both M3 and M4, suggesting that a team's decline in league standings by one position increases a player's exit probability by around $10 \%$. This is not surprising, especially in the wake of the relegation system where the two or three lowest ranked teams get demoted from the top European competitions, resulting in player exits unless they transfer to another top-level team. Playing for a better ranked team improves a player's probability of survival, whether he maintains a regular first-tea place on that team or he moves to a lower-ranked team in the top division; Average players from top teams have a higher probability of findin a spot on another top-division side in Europe than average players from lesser known teams, because their level of productivity is transferable and perhaps

87 Frick, Pietzner and Prinz (2007), as well as Radoman and Voia (2015, forthcoming) show that career duration of goaltenders is longer than for other positions considered, and forwards tend to have the shortest career duration.

88 Physical demands in soccer vary by position. For example, wingers and full-backs are dependent on speed and once that attribute deteriorates their exit probability should increase; Goaltenders, on the other hand, tend to play at an older age since their position is less physically demanding. In addition to being the main targets for opposition defences and all the injury risk that exposes them to, forwards also face the most public scrutiny as their performance is easily measured by goals scored. 
satisfactory for teams fightin for survival rather than battling for the league title or positions that lead to European competitions.

In terms of log-likelihood, model specificatio is improved when controlling for more covariates, with the highest improvement (against the M1 benchmark) being registered by M4 that accounts for nonlinear functional form of the team position covariate. Unsurprisingly, the AIC and BIC criteria, which is not reported, indicate M4 outperforms the other specification in terms of fit The largest improvement is registered when moving from M2 and controlling for team position in M3 and M4. There is a slight improvement in M4 versus M3, but the magnitude of improvement is significantl lower (15 versus 76) than when moving from M2 to M3. Baseline hazards of all specification are presented in Figure 2.6. M2's baseline is closest to the empirical hazard but both M3 and M4 mimic its shape even though M3 underestimates it and M4 slightly overestimates it. Hence, all three of these specification do a good job modelling the underlying duration dependence. Considering the log-likelihood of M3 and M4 is superior to M2, which indicates an improvement in model specificatio by controlling for team strength, their unsmoothed estimated hazards are presented against the empirical hazard in Figure 2.7. M3 mimics the shape of the empirical hazard better than M4 and is closer to it during the entire range of the graph, which indicates that M3 is superior in terms of graphical analysis. The graphical dominance of M3, as well as the almost negligible difference in log-likelihood with M4, make it the most reliable specificatio to use for this group According to 
the The Therneau-Grambsch test, the PH assumption of the Cox model holds up with at all reasonable levels of statistical significance both globally and for each covariate in M3 (and M4). The model's goodness of fi is tested using Cox-Snell residuals. Figure 2.8 demonstrates that M3 fit the data well, as the integrated hazard based on Cox-Snell residuals reverts to the 45 degree line. Therefore, M3 satisfie all of the empirical criteria for a robust Cox semiparametric model, and estimates the data well.

\subsubsection{Results for Post-Bosman entrants}

Table 2.5 presents the estimated results for all four specifications First-team appearances and minutes per game have a strong and statistically significan (at the $1 \%$ level) predictable negative effect on the hazard rate in all specifications The effect of international appearances is not statistically significan for M3, and is only significant at the $10 \%$ level in M4, while it is significan at the $1 \%$ and $5 \%$ level in M1 and M2, respectively; the significanc of the covariate effectively diminishes with improvements in model specification Interpreting from M4, making one more first team appearance reduces the exit probability for a player by $4.3 \%$, while playing one more minute per game reduces the exit probability of players by $1.05 \%$, and making one international appearance reduces the exit probability by $3.2 \%$. This is very interesting when comparing to the magnitude of the effect of the covariates in the Pre-Bosman results; While the magnitudes of the first-tea appearances effect on 
the hazard rate are similar, the magnitude of the minutes per game effect is more than double than in the Pre-Bosman results. This result lends support to theoretical predictions about higher productivity of the Post-Bosman entrants, and possibly higher overall talent level within this sub-sample; each additional minute spent on the fiel by these players seems to be more valuable and effective in the game of survival. Once again, we can see the magnitude of the international appearances effect decreasing as model specificatio is improved (moving from M1 to M4), but not to the same extent as in the Pre-Bosman results. Unlike in the Pre-Bosman results, moving from M1 to M3 and M4 does not result in noticeable changes in the impact of first-tea appearances on the hazard rate, while the same movement does increase the magnitude of minutes per game effect by $0.55 \%$.

Age of entry has the same predictable positive effect on the hazard rate, with each additional year increasing the exit probability by around $8.5 \%$ in M3 and M4. A player's positional role does not have a statistically significan effect once again, but nationality has a significan effect (at the $5 \%$ level) in all specification that account for it. This result differs from the Pre-Bosman group, and it could be due to the fact that most entrants prior to the Bosman ruling were UK citizens, while the ruling resulted in an influ of foreign players in the English Premier League making the data more reliable to assess the impact of nationality on player durations. Regardless, being a foreign national (either from an EU or non-EU country) reduced the exit probability of players by approximately $16-22 \%$ in M3 and M4. This could be due 
to the superior talent of foreign players as predicted by positive spillover theorists, or the mere perception that foreign players are more talented than domestic players. However, perception can only be deceiving when a player is signed and productivity, which includes talent, should re-surface as a key determinant for success and survival in a top-level league. Team position has a strong and statistically significan (at the $1 \%$ level) effect, indicating that a one position drop in a team's rankings increases a player's exit probability by $9.5 \%$.

Once again, the largest improvement (against the M1 benchmark) in model specification in terms of log likelihood, is observed in M4, which is also consistent with the AIC and BIC criteria. The improvement in M4 versus M3 is larger for this sub-sample than for the pre-Bosman players (30 versus 15$)$, while the improvement from M2 to M3 is about the same (around 70). Figure 2.9 (A) presents the baseline hazards for all specifications and we can see that they all model duration dependence well, particularly M4. Estimated hazards for best-fittin specifications M3 and M4, against the empirical hazard, are presented in Figure 2.9 (B). M4 mimics the empirical hazard better and closer than M3 and it appears to graphically outperform M3. Considering the graphical analysis and the log-likelihood measures, M4 was chosen as the most representative specificatio for this sub-sample. The PH assumption holds up for M4 (and M3) both globally and locally for each covariate. Satisfactory goodness of fi for M4 using Cox-Snell residuals is demonstrated in Figure 2.10. 


\subsubsection{Comparative Results}

The largest observable difference in the productivity measure results for the two groups is in the magnitude of the minutes per game covariate. For post-Bosman players, playing an additional 10 minutes per game decreases their exit probability by around $10.5 \%$ as opposed to around $4 \%$ for pre-Bosman entrants. This suggests that competition for places intensifie after the Bosman ruling and each minute spent on the pitch became increasingly important for a player's survival in top European leagues. This result is reasonable considering the effects of the Bosman ruling on the talent pool available to clubs to draw from, and the fact that the economic rents available to players in England were the highest in Europe making the Premier League more appealing to foreign players. Linking this result with the RD design sharp jump in this covariate, players in the post-Bosman period seem to be more productive, and measures of productivity (minutes per game in this case) become an increasingly important determinant in their probability of survival as competition for places heats up. This is indicative that total effort of these players increases as a result of added competition, which is consistent with contest theory predictions when we move to a more homogenous environment (in terms of talent). These conclusions seem that more reasonable considering that the English Premier League was the richest and fastest growing (in terms of revenues and player salaries) since the Bosman ruling and competition for places in England was certainly more heated than elsewhere. 
With respect to international appearances, the statistical significanc of its effect is negatively correlated with model specification controlling for player and team specifi covariates diminishes the significanc of international appearances, particularly for post-Bosman players. This is somewhat surprising because international appearances signal a highly rated and productive player, and one would expect this variable to have a significan impact on a player's exit probability. A possible explanation for this result is that many players from lesser known soccer countries were entering the top European leagues after the Bosman ruling, and the fact they represent their countries internationally does not separate them from average players in the league that their play club soccer in. The foreigner spots on a team's roster were much more valuable prior to the ruling due to the quota in place, meaning that English Premier League teams would only attempt to sign highly rated foreign nationals in line with their budget and other constraints. For example, an average player from the Czech Republic's national team would probably not be signed by a Premier League side pre-Bosman, but the same/similar player could be signed when the foreigner quota system was removed as a result of the ruling. This or any other lesser-known player (or from a lesser-known country) may not be any different in terms of talent and/or productivity than average English players, while making the English national team would be significantl harder. This would render the effect of international appearances insignifican for average foreign national players or even top players from low-ranked countries. This result is also consistent with the RD design results, where 
international appearances display a significan jump only at windows 1 and 2 around the Bosman ruling. As more and more foreigners entered the English Premier League after the implementation of the ruling, the effect of international appearances diminished and we don't see a discrete jump in this productivity parameter.

Figure 2.11 depicts the best-fittin smoothed empirical hazards ${ }^{89}$ for the two groups of players side-by-side. The estimated hazard for the pre-Bosman entrants is almost everywhere above its post-Bosman counterpart, which means that their probability of exit is higher at almost every point in their career. This result coincides with the empirical hazards and the theoretical predictions suggesting that post-Bosman entrants have a higher probability of winning the contest and surviving for a longer period of time in the elite European soccer markets. The implied higher probability of survival for post-Bosman entrants sheds some light on changes in the European soccer competitive environment arising from this policy change. The RD analysis clearly depicts that players entering the market after the ruling are generally more productive, which is shown to have a clear and statistically significan negative effect on the hazard rate in the models presented for both groups of players. This negative effect is amplifie for post-Bosman players examined. It is quite possible that this group of players is equipped with more innate skill (natural talent), that when combined with a high level of effort, results in increased productivity and survival in the top leagues. In addition, the pre-Bosman estimated hazard displays more variability

\footnotetext{
89 The results are consistent even when other representative specification are considered. Kernel-
} weighted local polynomial smoothing is performed for interpretation purposes. 
than the post-Bosman hazard function, which could be due to competitive pressures resulting from the ruling or that these players were more asymmetric in terms of natural talent. One cannot be sure whether the increased productivity stems more from differences in player ability or player effort, because these variables are diff cult to observe and/or measure but their combined effects should be representative of player productivity. The lack of a statistically significan presence of unobserved heterogeneity in both groups of players might suggest that differences in natural ability are not that great among the players examined, which is not surprising considering that players at that level represent the cream of the crop in terms of innate skill level or talent. ${ }^{90}$ Regardless of the underlying source, it is evident that players entering after the ruling exhibit higher productivity in soccer terms, and it is not surprising that their probability of survival in top European leagues is higher as well.

In addition to the side-by-side estimated hazards graph, statistical tests are performed for comparability and robustness purposes. A two-sample KolmogorovSmirnov test is performed and the results are displayed in Figure 2.12. The empirical distribution function (EDF) for M1 (pre-Bosman model) is larger (with the exception of very low hazards) and displays second-order stochastic dominance (SOSD) over the EDF of M2 (post-Bosman model), which means that M2 has lower hazards than M1. Furthermore, Cliff's delta test, that compares two non-normal distributions, is performed and the results are presented in Figure 2.13. Distribution X represents the

\footnotetext{
90 This is not to say that there aren't differences in talent between the two groups of players, because
} the heterogeneity tests among players are applicable within, and not between, the two groups. 
pre-Bosman group and distribution $\mathrm{Y}$ the post-Bosman group, and the ordinal analysis indicates that the probability that a random variable X's hazard is greater than randomly selected member from group Y, which is portrayed in the graph. Cliffs' delta provides a useful representation of effect size, and the estimated value of -0.39 indicates that group's X's hazards tend to be higher than group Y's, and that there exists some overlap between the distributions. ${ }^{91}$ Taken in totality, there is robust evidence that there are differences in the hazard distributions of the two groups examined, and that post-Bosman players have a higher probability of survival.

\subsection{Conclusion}

The Bosman ruling has received much attention from many different field due to its importance in re-shaping the competitive structure of European sports. This chapter accounts for the effects of the Bosman ruling within a contest theory framework. Moving to a more homogenous competitive environment increases total quality/productivity of new entrants, via increases in talent level and effort exerted by competing players, leading to higher probability of survival in top European leagues.

A uniquely collected data set is applied to test the research questions postulated. The results of the $\mathrm{RD}$ design nonparametric approach clearly indicate that there is a sharp jump in the two main productivity measures, appearances per season

91 Since Cliff's delta is an ordinal analysis, it is robust to normality violations and heterogeneity of variance. On the other hand, Second order stochastic dominance test takes the variance into account as is examplifie in Figure 19. Pre-Bosman hazards are far more variable than post-Bosman hazards. 
and minutes per game. The results of the survival analysis are robust and in support of the relevant theory that post-Bosman entrants have a higher probability of survival or winning a spot for a top-division club. Post-Bosman players seem to be endowed with more talent/ability and/or exert more effort than their pre-Bosman counterparts, at least at an average level. Clubs in the post-Bosman period had a much larger pool of talent to draw from, and the intensifie competition for first-tea places led to more effort being exerted by players in games and training, clearly definin a more efficien and productive environment. Controlling for player specifi and team characteristics is important in improving model specification

It is important to note that this analysis relies on a data set composed of only new entrants into the English Premier League, and cannot assess the impact of the Bosman ruling on all other incumbents in the league. Another limitation of the data is the lack of publicly available information on player salaries and other sources of income. An interesting extension to this chapter could be to examine the impact of the Bosman ruling on a sub-group of players based on their source of entry. For example, the impact of the ruling on club owned youth academies and the career success of the players they produce warrants additional research. Assessing the strength of youth academies and domestically sourced players versus foreign sourced players can address some of the public debates of soccer non-academic experts regarding suggested protectionist policies in European soccer, particularly in England. In addition, performing a similar analysis for other European competitions can be fruitful 
to assess whether there is consistency in the results across the top EU leagues. Another interesting avenue for future research could be to estimate the cut-off point for necessary talent/effort level to enter the top level leagues in Europe pre and post the Bosman ruling, if such data can be collected with confidence 


\subsection{Tables and Figures}

Table 2.1: Player Entries by Bosman and Source

\begin{tabular}{c|cc|c}
\hline \hline Player Source & Pre-Bosman & Post-Bosman & Total \\
\hline Domestic & 130 & 110 & 240 \\
Youth & 148 & 152 & 300 \\
Foreign & 79 & 242 & 321 \\
\hline Total & 357 & 504 & 861 \\
\hline
\end{tabular}

Table 2.2: Sample Summary Statistics

\begin{tabular}{c|cccccc}
\hline \hline Bosman & Apps & IntApps & Age & TeamPos & Player Cost & ForShare \\
\hline Pre-Bosman & 18.8 & 0.8 & 25.4 & 10.8 & 1.22 Million & 32.2 \\
\hline St. Deviation & 11.9 & 2.1 & 4.4 & 6.1 & 3.04 & 2.5 \\
\hline Minimum & 1 & 0 & 16 & 1 & 0 & 30.3 \\
\hline Maximum & 42 & 13 & 39 & 22 & 40.5 & 37.1 \\
\hline Post-Bosman & 19.7 & 1.4 & 26.4 & 10.4 & 1.95 & 49.1 \\
\hline St. Deviation & 11.5 & 2.9 & 4.6 & 5.7 & 3.7 & 4.3 \\
\hline Minimum & 1 & 0 & 16 & 1 & 0 & 37.1 \\
\hline Maximum & 38 & 16 & 41 & 20 & 35.2 & 52.4 \\
\hline Total & 19.3 & 1.1 & 26 & 10.6 & 1.66 & 42.2 \\
\hline St. Deviation & 11.7 & 2.6 & 4.5 & 5.8 & 3.47 & 9.1 \\
\hline Minimum & 1 & 0 & 16 & 1 & 0 & 30.3 \\
\hline Maximum & 42 & 16 & 41 & 22 & 40.5 & 52.4 \\
\hline
\end{tabular}


Table 2.3: Treatment Effects

\begin{tabular}{c|ccccc}
\hline \hline Treatment & Window & Outcome & Coefficien & Standard Error & p-value \\
\hline \hline Bosman & 1 year & $\mathrm{mpg}$ & 4.68 & 1.41 & 0.001 \\
Bosman & 2 years & $\mathrm{mpg}$ & 6.36 & 2.22 & 0.004 \\
Bosman & 3 years & $\mathrm{mpg}$ & 7.04 & 1.79 & 0.000 \\
Bosman & all years & $\mathrm{mpg}$ & 6.38 & 1.54 & 0.000 \\
\hline Bosman & 1 year & apps & 2.08 & 0.76 & 0.006 \\
Bosman & 2 years & apps & 2.76 & 1.19 & 0.021 \\
Bosman & 3 years & apps & 2.64 & 0.96 & 0.006 \\
Bosman & all years & apps & 2.19 & 0.83 & 0.008 \\
\hline Bosman & 1 year & intapps & 0.45 & 0.17 & 0.009 \\
Bosman & 2 years & intapps & 0.74 & 0.27 & 0.006 \\
Bosman & 3 years & intapps & 0.33 & 0.22 & 0.134 \\
Bosman & all years & intapps & 0.13 & 0.19 & 0.505 \\
\hline Bosman & 1 year & intmpg & 5.32 & 2.06 & 0.003 \\
Bosman & 2 years & intmpg & 9.68 & 3.27 & 0.010 \\
Bosman & 3 years & intmpg & 4.24 & 2.62 & 0.106 \\
Bosman & all years & intmpg & 1.41 & 2.27 & 0.535 \\
\hline
\end{tabular}

Table 2.4: Cox Pre-Bosman Results

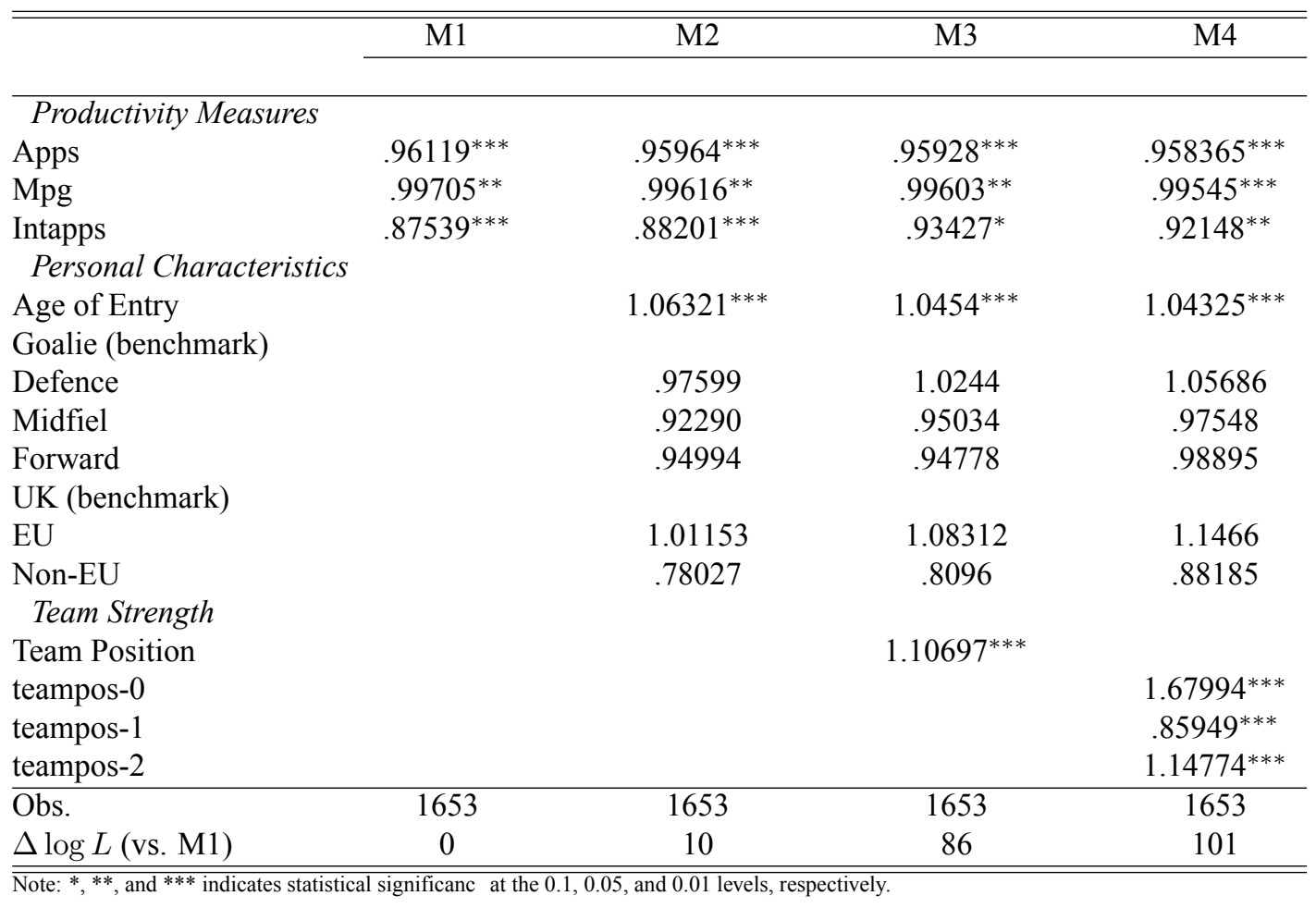


Table 2.5: Cox Post-Bosman Results

\begin{tabular}{|c|c|c|c|c|}
\hline & M1 & M2 & M3 & M4 \\
\hline \multicolumn{5}{|c|}{ Productivity Measures } \\
\hline Apps & $.95758^{* * *}$ & $.95775^{* * *}$ & $.95886^{* * *}$ & $.95793^{* * *}$ \\
\hline MPG & $.99506^{* * *}$ & $.993^{* * *}$ & $.98937^{* * *}$ & $.98954^{* * *}$ \\
\hline Intapps & $.93348^{* * *}$ & $.94985^{* *}$ & .97293 & $.96792^{*}$ \\
\hline \multicolumn{5}{|c|}{ Personal Characteristics } \\
\hline Age of Entry & & $1.09702^{* * *}$ & $1.0877^{* * *}$ & $1.085^{* * *}$ \\
\hline \multicolumn{5}{|l|}{ Goalie } \\
\hline Defence & & .94894 & .85901 & .87785 \\
\hline Midfiel & & .98757 & .89032 & .93060 \\
\hline \multicolumn{4}{|l|}{ UK } & .85608 \\
\hline EU & & $.65924^{* * *}$ & $.83718^{* *}$ & $.84945^{* *}$ \\
\hline $\begin{array}{l}\text { Non-EU } \\
\text { Team Strength }\end{array}$ & & $.67229^{* * *}$ & $.76206^{* * *}$ & $.78536^{* *}$ \\
\hline Team Position & & & $1.09509^{* * *}$ & \\
\hline teampos-0 & & & & $1.50665^{* * *}$ \\
\hline teampos-1 & & & & $.82412^{* * *}$ \\
\hline teampos-2 & & & & $1.19509^{* * *}$ \\
\hline Obs. & 2403 & 2403 & 2403 & 2403 \\
\hline$\Delta \log L$ (vs. M1) & 0 & 32 & 108 & 138 \\
\hline
\end{tabular}

Note: ${ }^{*}, *$, and ${ }^{* * *}$ indicates statistical significanc at the $0.1,0.05$, and 0.01 levels, respectively. 


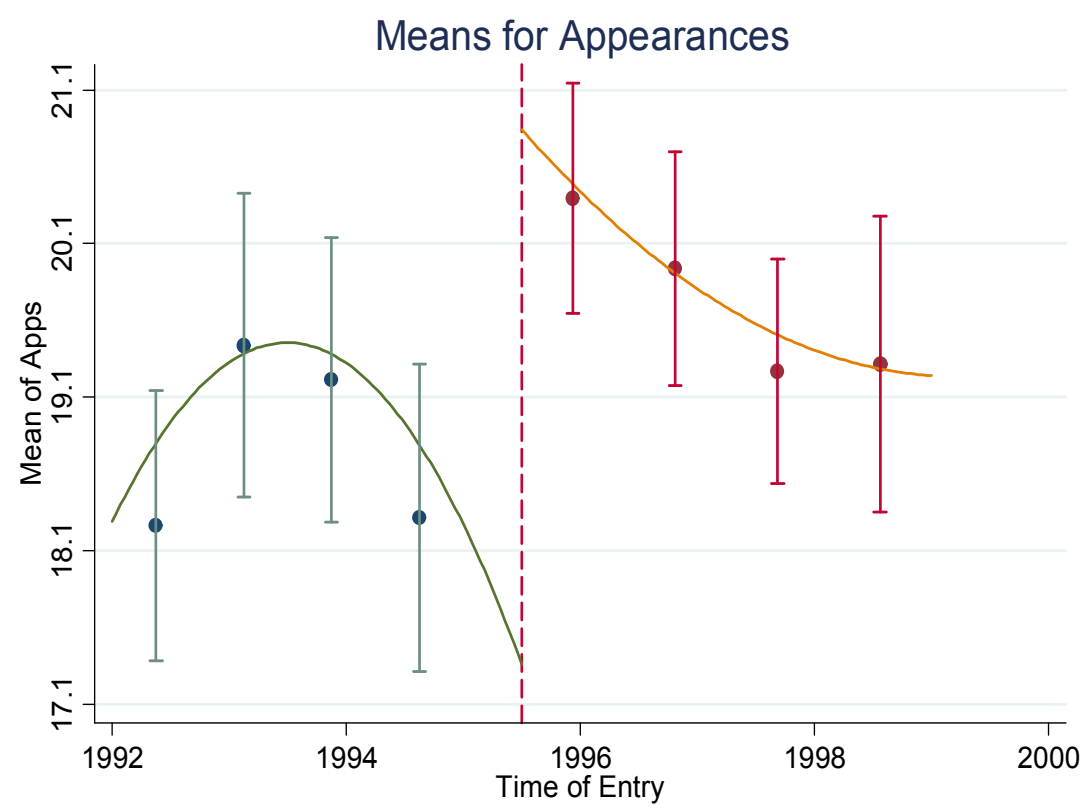

Figure 2.1: Graph of bin means for Apps

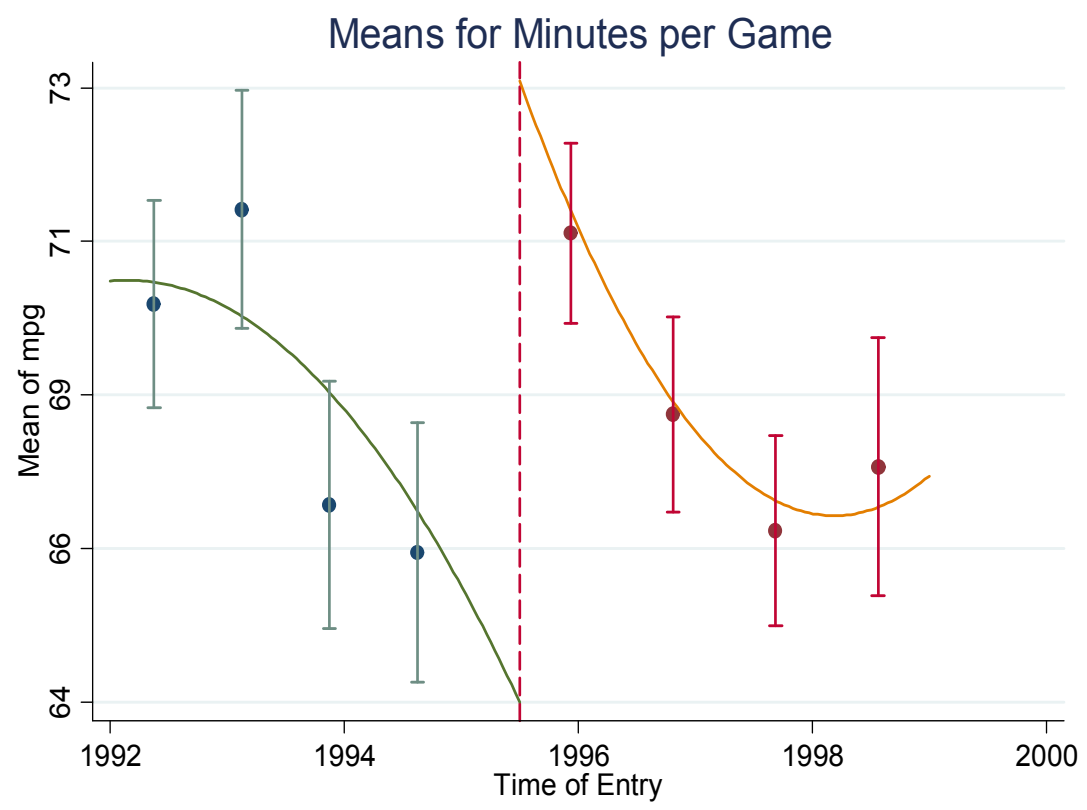

Figure 2.2: Graph of bin means for MPG 

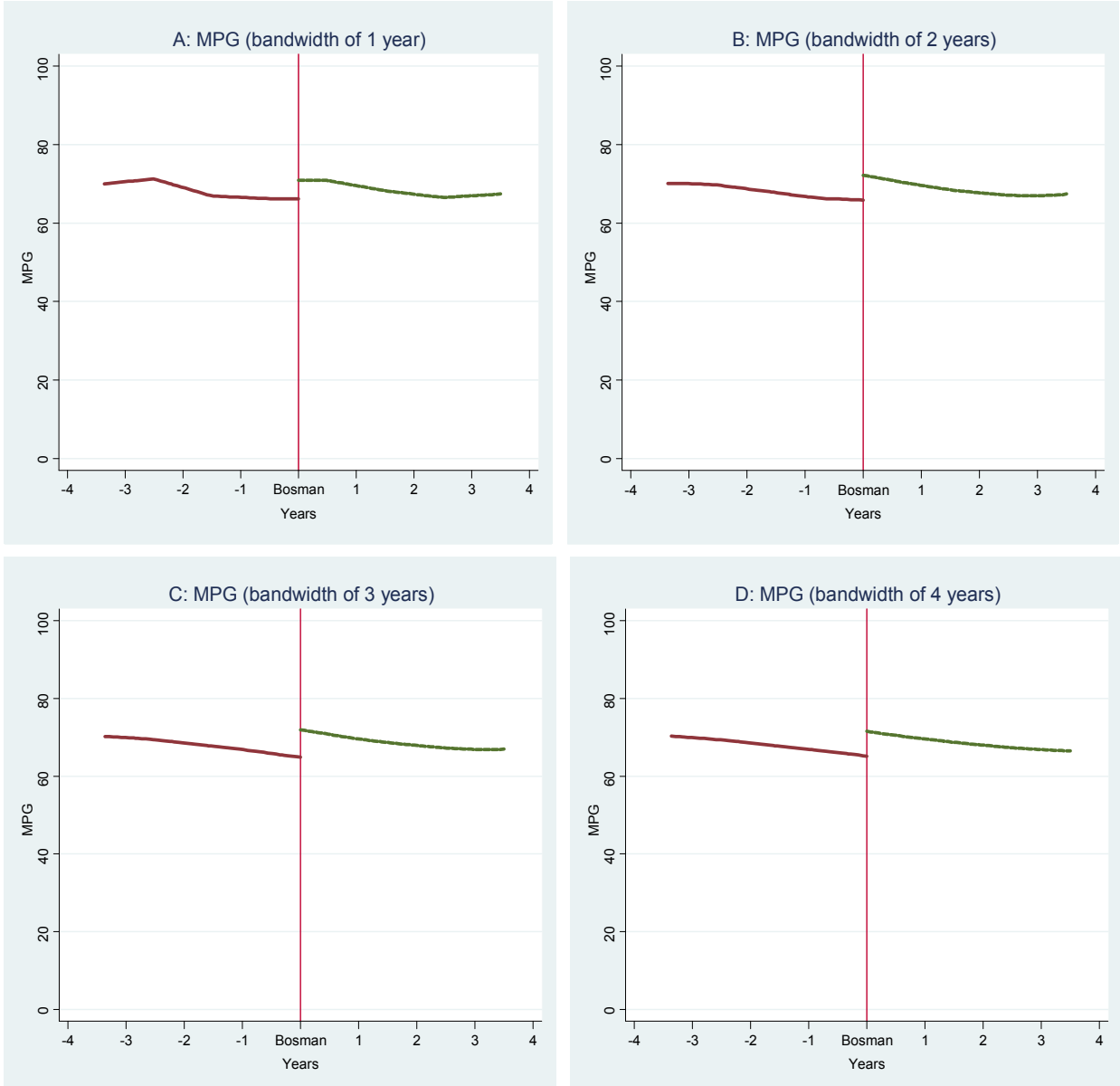

Figure 2.3: (A) Mpg for 1 year bandwidth, (B) Mpg for 2 year bandwidth, (C) Mpg for 3 year bandwidth, (D) Mpg for 4 year bandwidth 

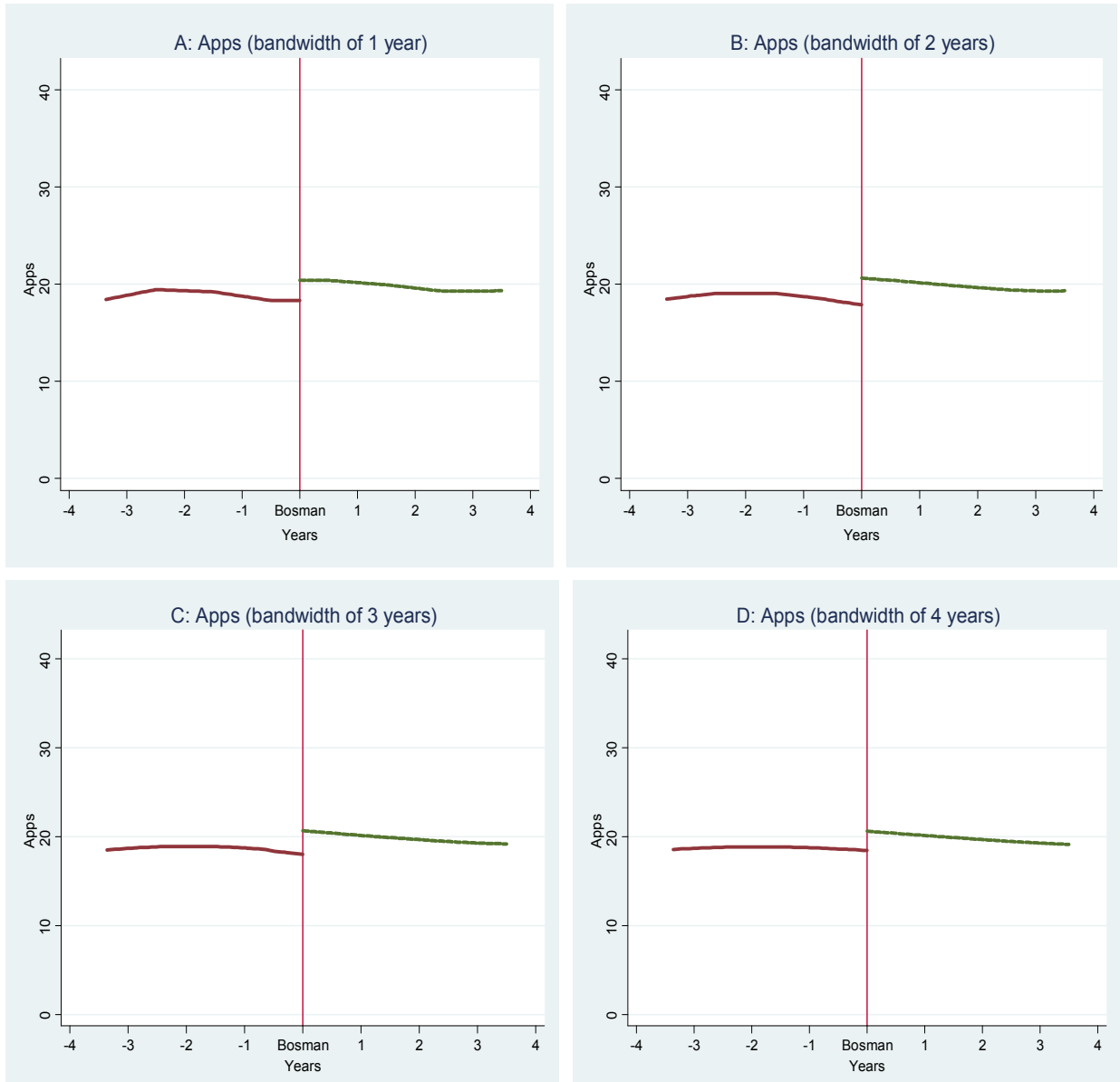

Figure 2.4: (A) Apps for 1 year bandwidth, (B) Apps for 2 year bandwidth, (C) Apps for 3 year bandwidth, (D) Apps for 4 year bandwidth 


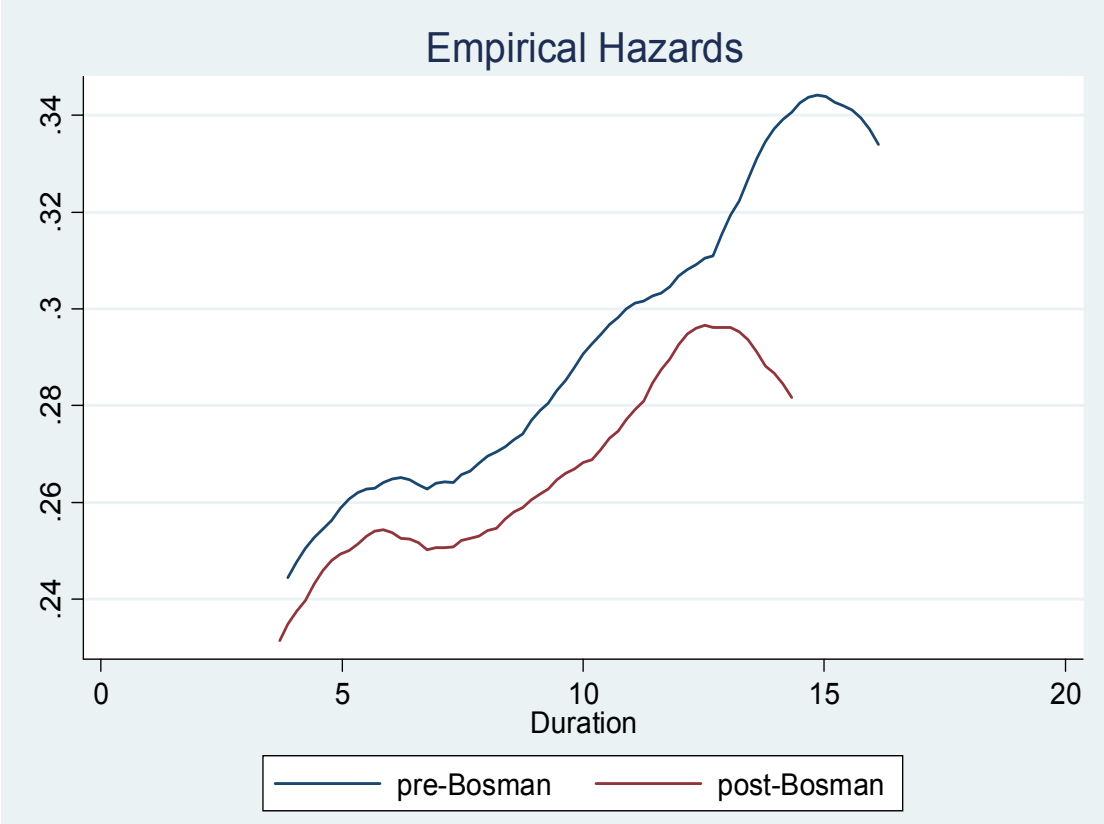

Figure 2.5: Empirical Hazards for Both Groups

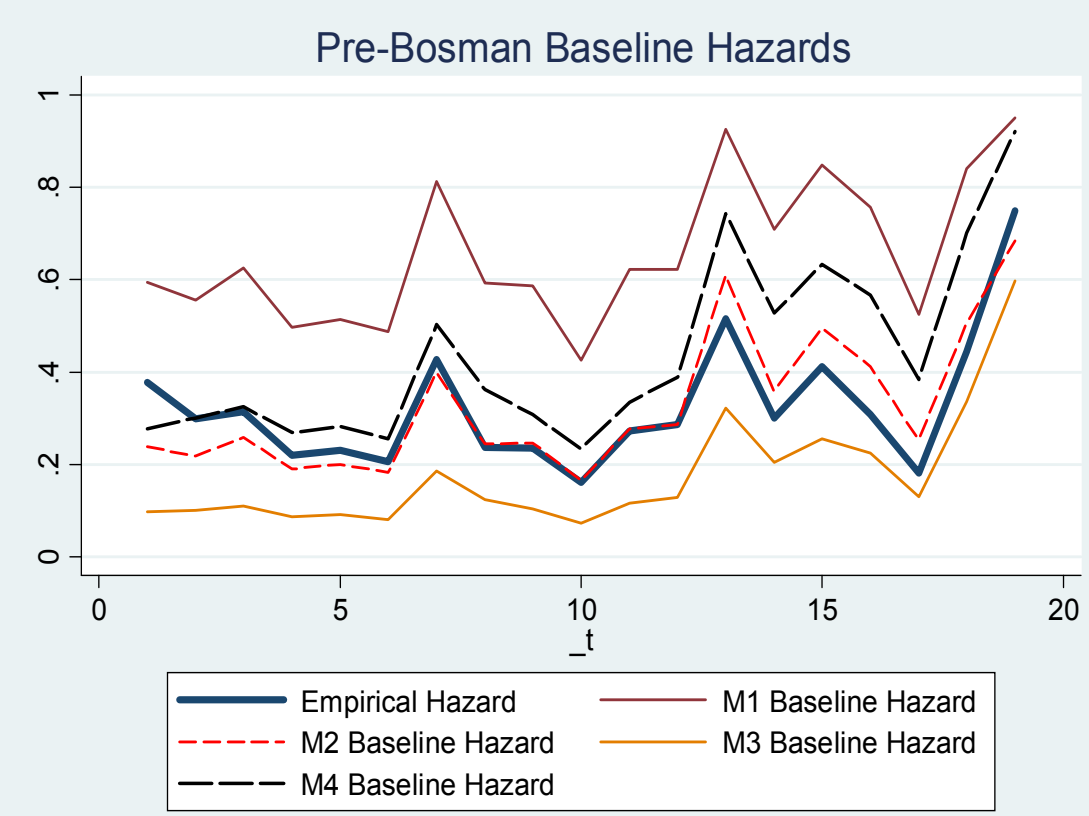

Figure 2.6: Pre-Bosman Baseline Hazards 


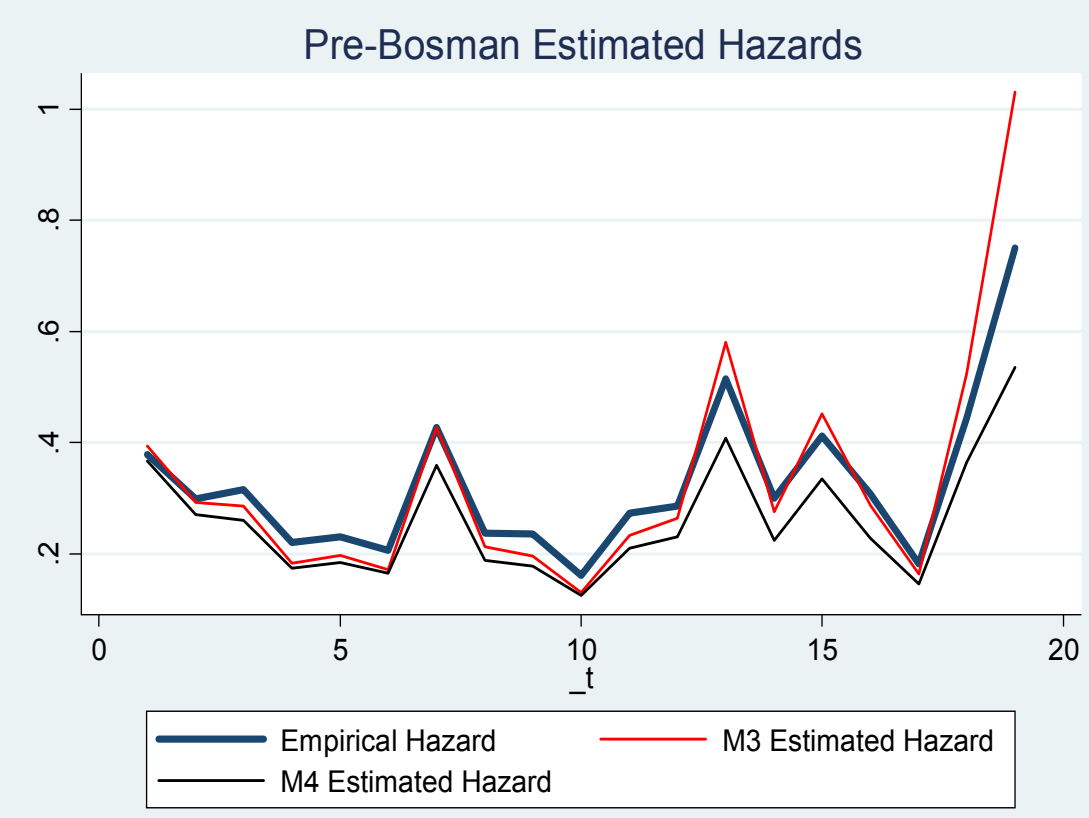

Figure 2.7: Pre-Bosman Estimated Hazards

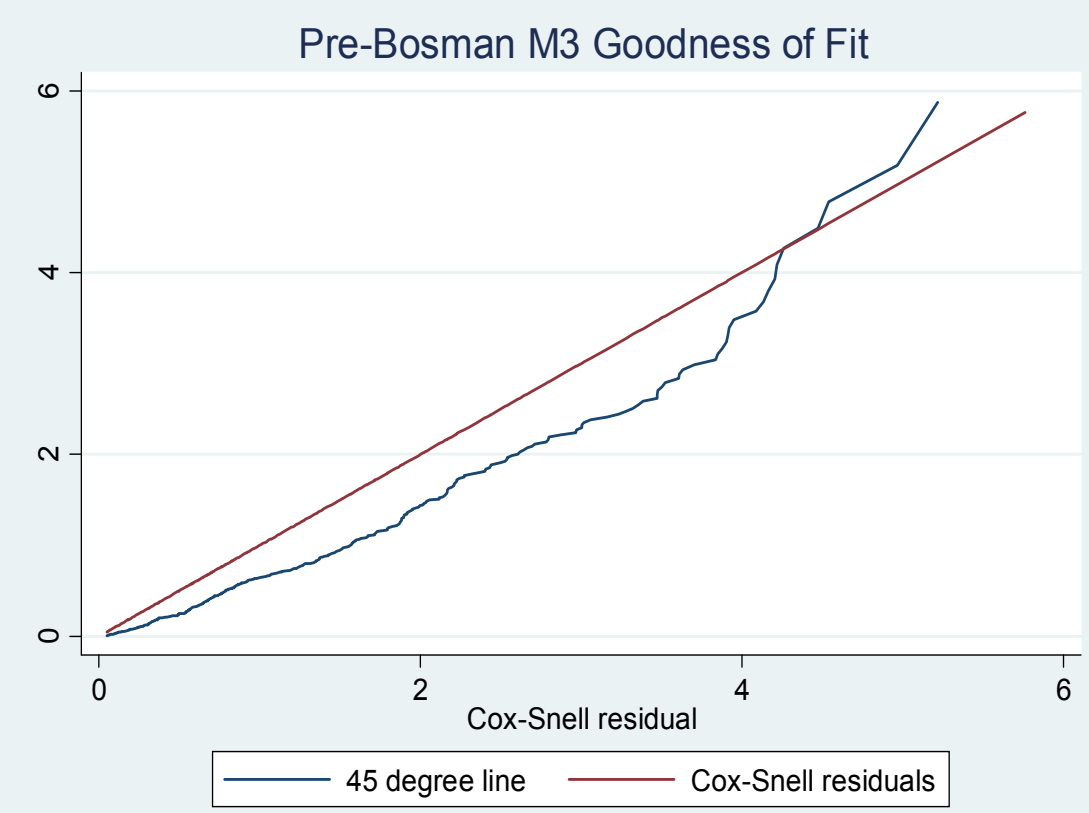

Figure 2.8: Pre-Bosman Goodness of Fit 

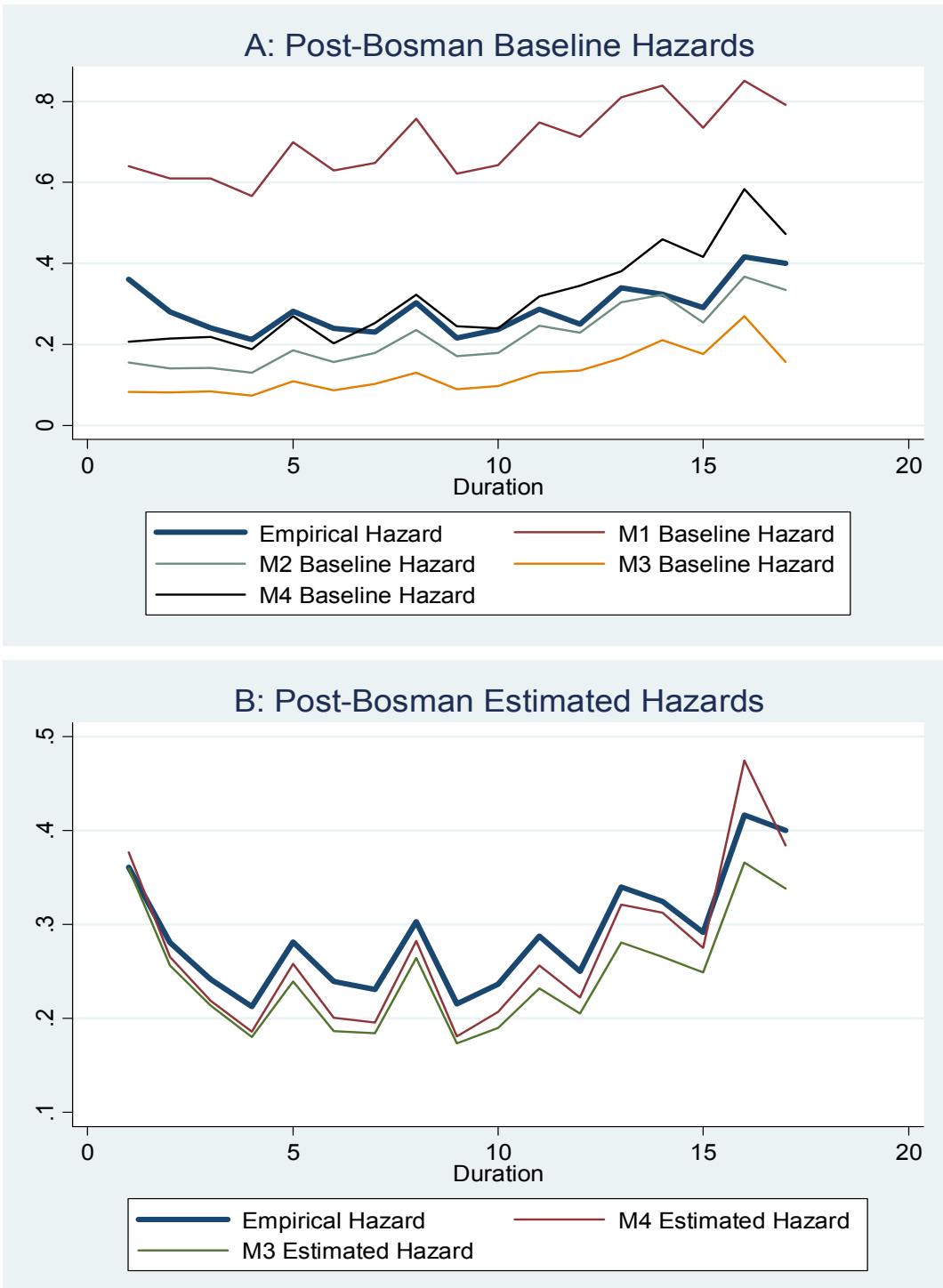

Figure 2.9: (A) Post-Bosman Baseline Hazards, (B) Post-Bosman Estimated Hazards 


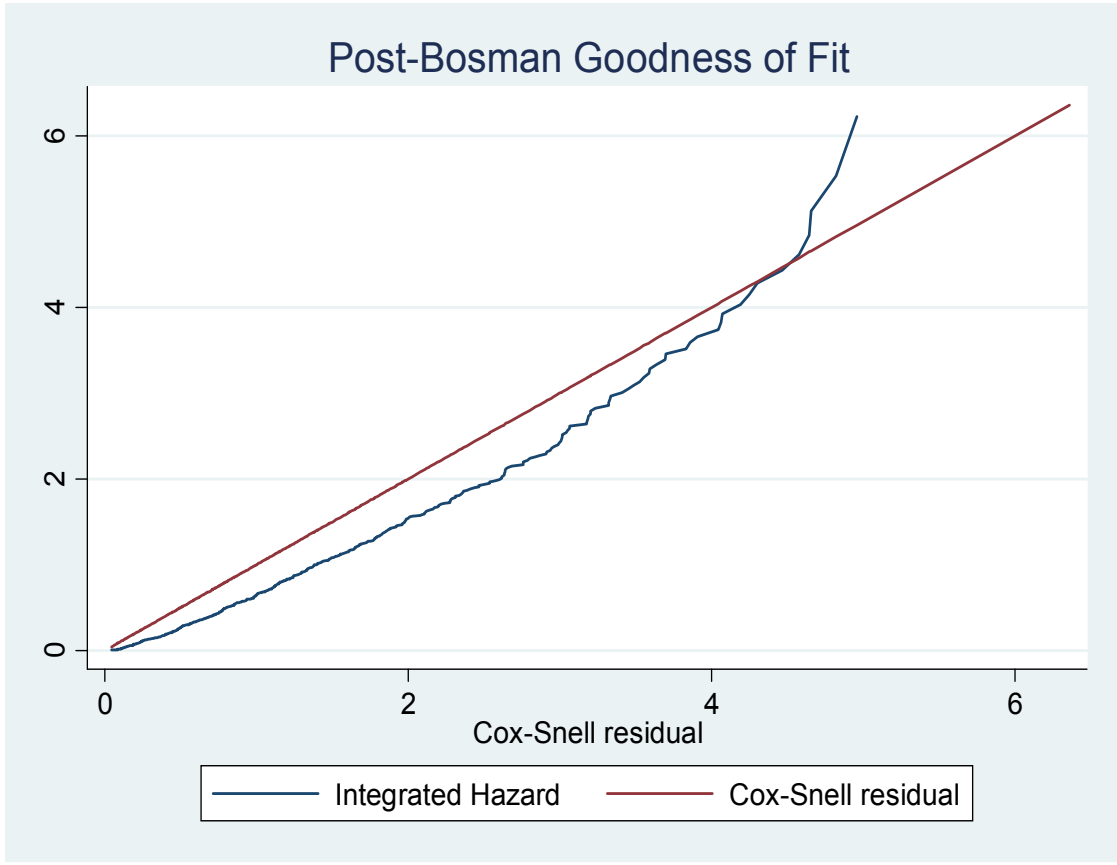

Figure 2.10: Post-Bosman Goodness of Fit Test

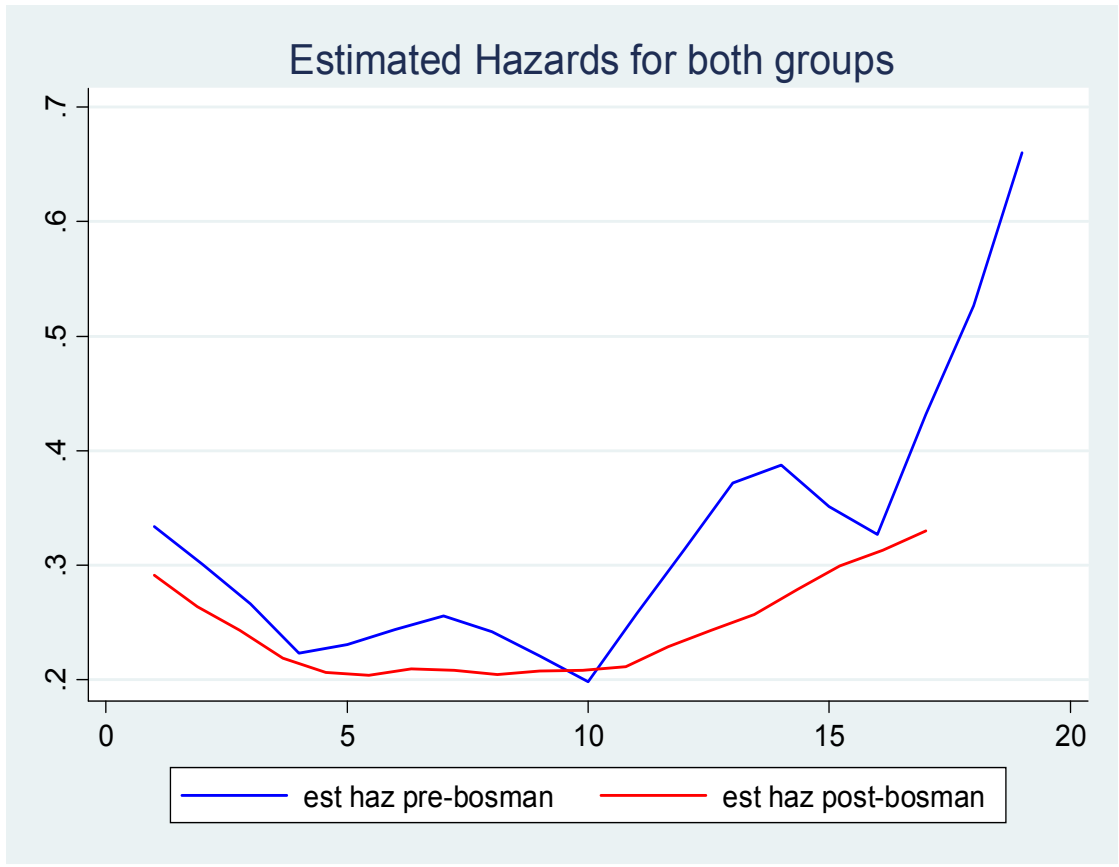

Figure 2.11: Estimated Hazards for Both Groups 


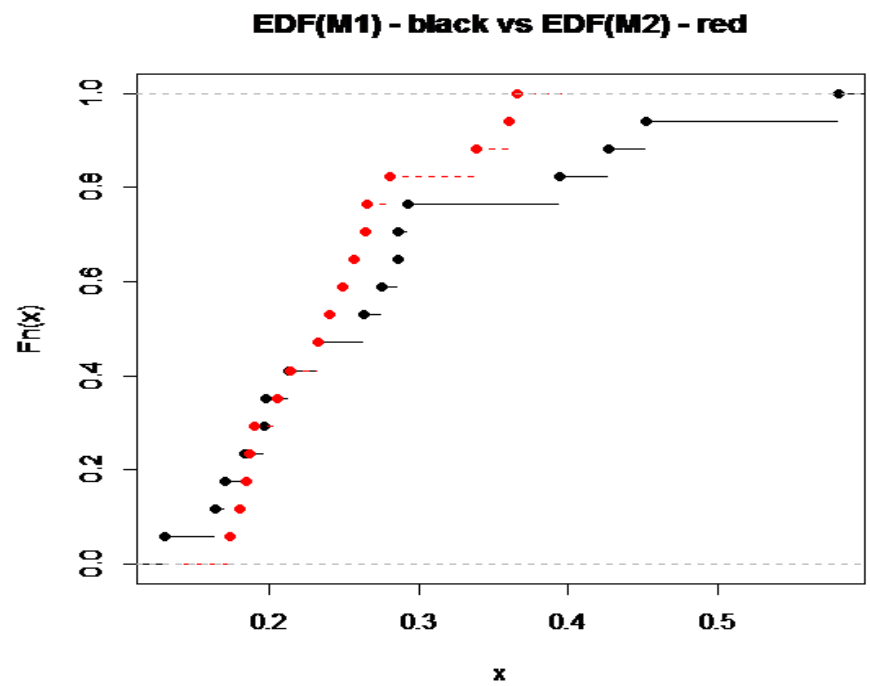

Figure 2.12: Kolmogorov-Smirnov Test for Stochastic Dominance

Ciffis delta (combined) \& 95\% Confidence Interval (2-tailed)

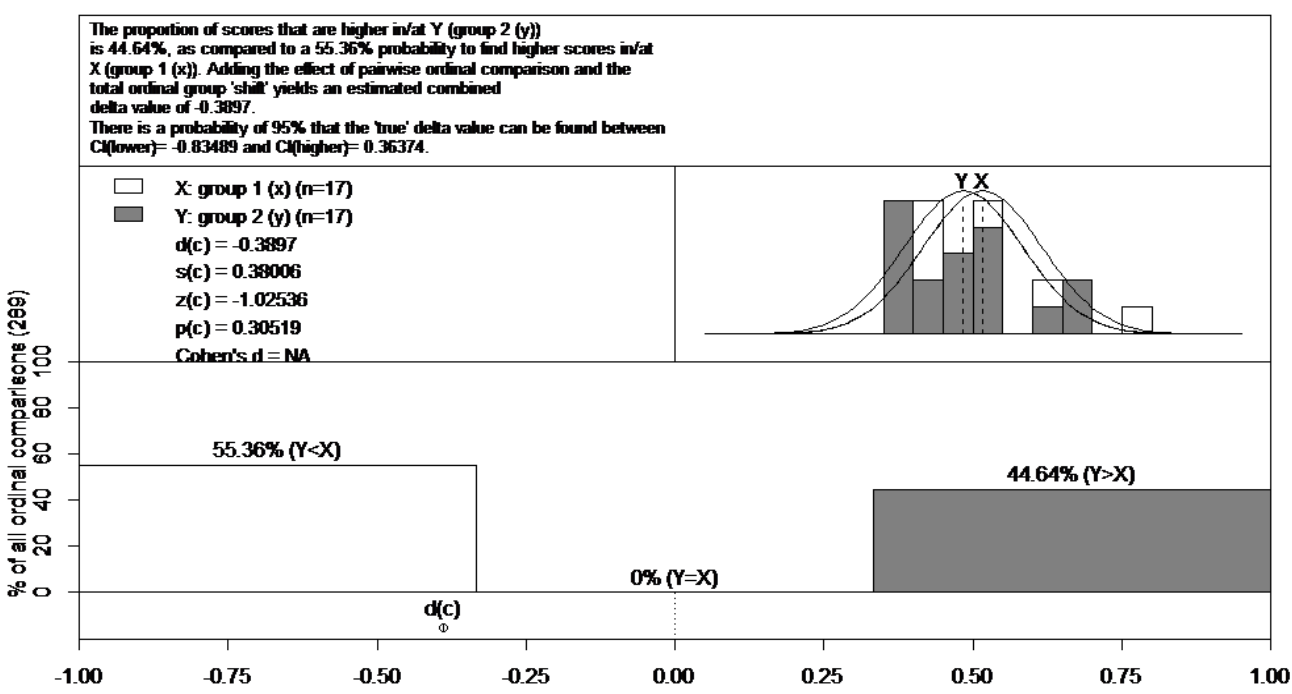

Figure 2.13: Cliff's Delta Analysis 


\section{Chapter 3 \\ Internal Promotion in Competitive Sports: Evidence from the English Premier League}

\subsection{Introduction}

Internal promotion or external hiring is a dilemma faced by firm operating in many distinct sectors of the economy. Heavy investment in training exposes firm to the risk of losing the talented employees they trained to competitors without exhausting the full benefit of their investment. External hiring has its own risks and uncertainties surrounding the worker's ability to integrate effectively within the new firm Unsurprisingly, this economic dilemma is encountered in professional sports around the globe, particularly in European soccer. The soccer clubs in Europe make a spectrum of investment decisions that can significantl affect their long-term success and financia stability, but very few are as important as deciding whether to invest heavily in their youth academies or resort to the transfer market to recruit new players. Providing firs-tea opportunities to players developed in the youth academy/program can be viewed as promoting from within, while hiring first-tea players from other sources can be viewed as external hiring. The increasing importance of this dilemma is evident in the current market where transfer fees for star players 
are in excess of 100 million euros $;{ }^{92}$ building a team by relying solely on the transfer market is unimaginable even for the wealthiest clubs. Developing and nurturing of home-grown talent presents an alternative strategy that could be far less costly but possibly more uncertain in terms of attaining sufficientl talented players in the long-run. Most clubs these days, particularly in top European competitions, rely on both streams for bringing in new players but the weighting of investment in these two alternatives varies by club.

There are significan risks associated with both options that vary by club size and stature, which are further complicated by the transfer market for players under contract in Europe. For example, and much like in any other sector of the economy, soccer clubs may lose the youth players they developed to other clubs, which could be troubling if their goal was to keep them and integrate them in the first-team On the other hand, clubs can invest in youth academies in order to generate profi from the sale of these players in the transfer market if their focus is on profi maximization in the short-run, rather than on win maximization. Bringing in players from external sources carries the inherent risk of them not being able to fi in to the new team and system, which can stem from their personal characteristics to professional ones. The scale of a club and financia resources available are an important determinant in these decisions as well. Larger clubs have more options available to them in this regard, while smaller clubs do not have the financia ability to compete for talent in the

92 Gareth Bale was sold by Tottenham to Real Madrid for a fee that exceeded 100 million euros. Luis Suarez was transferred from Liverpool to Barcelona for over 80 million euros, while James Rodrigues went from Monaco to Real Madrid for around 90 million euros in the summer of 2014. 
transfer market, which might dictate their decision to focus on the youth development channel.

The Bosman ruling (December, 1995) in Europe certainly added an additional level of complexity in these decisions faced by clubs. The talent pool available to clubs expanded significantl as a result of the elimination of the foreign player quota for players originating from EU countries. In addition, players that were out of contract at their club were allowed to move for free (without any transfer fees) to another club, which potentially exposed clubs to greater risk in terms of losing the players they developed through the youth system without any compensation. All of these changes brought interesting dynamics to the internal vs. external hiring decisions of soccer clubs. Therefore, examining the hiring patterns of top-division clubs presents a question worth exploring, especially in the years surrounding the Bosman ruling.

Certain clubs have built a reputation of youth development and training that emphasizes their focus and investment in this channel. Examples of world renowned soccer academies include the likes of FC Barcelona and Real Madrid in Spain, Bayern Munich in Germany, Ajax in Holland, Udinese in Italy, Sporting Lisbon in Portugal, Arsenal in England, etc. The focus of this analysis is on the English Premier League, where some of these most reputable youth academies and clubs reside. Chapter 1 analyzed the historical performance of youth players from 16 top-level English clubs in terms of their career duration in top European leagues. This chapter uses that information to create a unique index for youth development reputation 
of the clubs examined in the current study. In addition to this index and the original data set, this chapter creates a link between existing internal vs. external hiring labor literature and a club's decision to hire new players externally or resort to their internal resources (their youth system). The index establishes an inherent ranking in terms of reputation of youth programs among English Premier League clubs, and the empirical analysis supports the theoretical assumptions that more reputable clubs provide more opportunities to their youth players. The behaviour of clubs is analyzed in a specifi period that accounts for a major institutional change in the sport, the Bosman ruling. The club ordered results are consistent when controlling for the ruling, even though youth sourced players were negatively affected by this change in favor of foreign player hires. Dominant, or more competitive, clubs (in terms of league performance) have a higher probability of selecting foreign players in available first-tea slots, which is amplifie after the Bosman ruling. This result signals their superior resources in the augmentation of their player-recruitment operations after the institutional changes freed up the EU labor market for players. Furthermore, survival analysis is performed that validates the index club reputation when it comes to career duration of youth products at the top level of European soccer. One interesting result suggests that unobserved heterogeneity is not present at player level, while it becomes a significan factor at the youth parent club level. This indicates that there is a unique "schooling" aspect to each youth program that equips players with necessary skills for a lengthy career at the top level. 
The remainder of the chapter is structured as follows: Section 2 outlines the theoretical motivation for the research task at hand, section 3 provides an overview of the data generating process and an overall summary of the data, section 4 describes the sequential empirical methodology employed, section 5 provides the results for the firs step independent probit estimation, section 6 provides the results of the second step bivariate probit estimation, section 7 describes the results of the survival analysis stage of the empirical methodology, and section 8 concludes the chapter.

\subsection{Motivation}

Applying certain labor market theory can aid in explaining the change in the English Premier League competitive environment brought upon by this institutional change, and can explain the different behavior of clubs in these circumstances. One particular angle of labor economics is worth exploring from a sports economics context, particularly European soccer; internal promotion or hiring from external sources. A fundamental question is why do firm invest in training of employees when they might lose them to competitors later on? A similar question can be asked in European soccer regarding club investments in their youth academies when first-tea spaces are very limited and they risk losing quality players that they trained. ${ }^{93}$ Waldman (2013) provides a survey of economic literature on this issue, focusing on tying economic

93 One recent example is Paul Pogba, a player brought up through Manchester United's youth system but who joined Juventus on a almost free transfer when his youth contract expired. His current value exceeds 60 million euros, and Manchester United will not benefi at all from his talents or future transfers. 
theory with empirical evidence. Oyer (2007) argues that there is an insider advantage in getting tenure for economic academic positions in universities (outside the top-ten economic institutions), even though external candidates are generally more productive.

DeVaro and Morita (2013) present a theoretical and empirical analysis of internal promotion versus external recruitment for managerial positions, using a cross section of British firms They argue that a firm s decision to hire managers internally versus externally is influence by the size and the quality of the talent pool available at the lower ranks, which is inherently determined by the firm s own hiring decisions. They argue that a fir with higher return to managerial capability tries harder to fil the management positions by hiring internally, and such firm higher more subordinates at lower levels and provide them with more general training to increase the number of workers with managerial potential. Therefore, these "bottom-heavy" employers have a greater probability of fillin their management positions through an internal hierarchy. According to the authors, their theory potentially explains common empirical finding that large firm are more likely to hire CEOs internally than small firms This logic is applicable in European soccer and explains why certain clubs invest more heavily in their youth training programs, as well as coaching and scouting at that level. Clubs that have built-up a reputation and a track record in terms of youth training should have a higher probability of fillin first-tea spaces via internal sources, particularly from its own youth academy. Hence, this type of 
labor market theory can be examined through the sports economics perspective by examining English Premier League clubs that endogenously determine their own hierarchical structure and weight of investment in youth training.

This chapter builds on the results from chapter 1 and attempts to assess whether clubs with high-performing youth programs maintain their historical reputation and focus on youth players in a narrower time span. What makes this analysis more interesting is that the time span examined encompasses the ever important Bosman ruling, and allows for testing its effects on the hiring behavior of English Premier league clubs. The Bosman ruling represents one of the most significan external shocks in the history of European sports. It freed up the European sports labor market by removing restrictive policies in player contracts and for player movement within the EU. Its effects and implications have been the subject of analysis of many academic studies. The English Premier league is considered to be the richest among top European leagues in terms of club turnover and player salaries, especially after the Bosman ruling. ${ }^{94}$ This ensures a highly competitive environment where top players from around the globe compete for first-tea spots and clubs that have an abundance of choice in their scouting efforts. The high salaries in the league attract world-class players, and the financia resources available to clubs do not constrain them in their player recruitment efforts as much as in other European leagues.

94 Please refer to Chapter 2 for more details on the growing trends in European football. 
In essence, the empirical analysis will attempt to address some important questions that are applicable in a wider labor economics context: (i) Do more youthreputable (or bottom-heavy) clubs give greater opportunities to youth players in their first-team? (ii) How is their behavior affected by significan external shocks, such as institutional changes brought upon by the Bosman case?, (iii) Are smaller clubs able to compete with larger clubs in acquiring foreign talent, or does the Bosman ruling inherently change the focus on internal versus external promotion for clubs of different scale?

\subsection{Data and Summary Statistics}

The data set is composed of 857 players that entered the English Premier League for the firs time ${ }^{95}$ from the $1992 / 1993$ to the $1999 / 2000$ season. Relevant information was gathered from publicly available internet sources, but mainly from the following sites: http://www.worldfootball.net/, http://www.transfermarkt.co.uk/en, and http://www.soccerbase.com. The beginning of the data collection period coincides with the establishment of the Premier League, and the seasons examined incorporate the effects of the Bosman ruling, which occurred almost midway through the analysis period. Players are distinguished by source of entry within three categories: players coming from a club's youth program ("Youth"), players sourced from lower

95 In fact, the data consists of all new entrants in the Premier League that made at least one appearance in the firs team for the club that hired them. This is particularly important when it comes to youth player because clubs have a number of youth players under contract at any time, but only a few are given opportunities in the first-tea so this is a better measure with this regard. 
level ${ }^{96}$ domestic leagues ("Domestic"), and players sourced from foreign leagues ("Foreign"). The data collected for each player also includes: club/team that hired the player and the team's table position/ranking in the league at that point, transfer fee paid for the player's rights (applicable only if players was sourced externally), player's name, position, age, nationality, whether the player entered before/after the Bosman ruling, international experience of player before entry, ${ }^{97}$ experience in toplevel leagues outside of England, ${ }^{98}$ and the share of foreign players in the Premier League for each season. In addition, an index of youth training reputation is created using the results from the firs chapter $^{99}$ in order to examine the consistency of the results and reputations of these clubs during the period affected by the Bosman ruling. The firs chapter is based on 16 clubs and the ordinal index applied in this chapter is generated as follows: category 1 clubs includes the top f ve clubs in terms of career duration of the players brought up through their youth system, ${ }^{100}$ category 2 clubs includes youth programs ranked from $6-10,{ }^{101}$ category 3 clubs contain the

96 Lower level leagues in England include: The Championship, League One, League Two, etc.

97 Data is recorded if a player represented his country at junior or senior levels. If the player represented his country at all levels, only the senior level is recorded because it is the highest achievement attainable at the international level. Obviously, youth players generally do not represent their countries at the senior level before they make a first-tea appearance for their club so their highest attainable level is the junior level at that stage of their career.

98 Players are differentiated by their experience in a top-level league from Europe versus experience in any other top-level league outside these leagues.

99 The data set from the firs chapter encompasses a much longer period of time and only a specifi selection of clubs, which differs significantl from the data in chapter 2 . The overlap of players is not very significant and there are different clubs involved that should not guarantee redundant results.

100 The highest performing f ve clubs (category 1) in all relevant model specification in Radoman and Voia (2015) are: Arsenal, Liverpool, Tottenham, Leeds United, and West Ham.

101 The clubs in this category include: Everton, Chelsea, Newcastle, Nottingham Forrest, and West Brom. 
remaining clubs of the ones examined, ${ }^{102}$ and category 4 includes all clubs not examined. In addition, a separate youth ranking index is created that includes Manchester United (Man U) in the firs category of youth programs. ${ }^{103}$ The main reason behind this is that Man $U$ changed their focus on youth development before and during the study period, especially since the arrival of their legendary manager, Sir Alex Ferguson in the late $80 \mathrm{~s}$, and their historical ranking is not consistent with the current study period. In fact, Man U's golden generation of youth players entered the market during this particular analysis period, so it could be problematic to group them in a lower-ranked category. Another index is created (Team rank) to rank the clubs by their position in the table to establish a certain grouping and differences in club stature. Clubs ranked in the top 7 positions of the Premier league table are grouped in category 1 , while clubs ranked in positions $8-15$ are grouped in category 2 , and the bottom 5 ranked clubs are considered category 3 clubs. The logic behind the ranking is that the top 7 places in the league lead to places in European competitions, which are generally occupied by the most competitive clubs in the league; the bottom 5 clubs are typically the ones fightin for survival in the league by avoiding relegation, and the rest of the clubs can be considered mid-ranking clubs.

102 This category includes the lowest ranked from the 16 clubs examined: Manchester United, Manchester City, Sheffiel Wednesday, Coventry City, Southampton, and Aston Villa.

103 The original index created ranks Man U in category 3 youth programs based on historical performance that does not account for this particular study period with sufficien weight. Therefore, the index is modifie to include Man $\mathrm{U}$ in the top category but as we will see later, the results are not dependent on the index applied. 
Out of the total of 857 players, 357 entered in the pre-Bosman period, while 500 entered in the post-Bosman period. These entries can be broken down further by player source; In the pre-Bosman period there were a total of 131 Domestic entries, 147 Youth player entries, and 79 player entries from Foreign sources, while in the post-Bosman period there were 110 Domestic entries, 149 Youth entries, and 241 Foreign source entries. Unsurprisingly, the number of foreign player entries increased significantl (more than tripled) as a result of the removal of the foreign player quota and foreign player entries represented nearly half of all new entrants to the Premier League in the post-Bosman period versus close to $25 \%$ in the preBosman period. It appears that players sourced from Domestic sources suffered the most as a result of the Bosman ruling in terms of opportunities in the Premier League; they represented $37 \%$ of player entries in the pre-Bosman period versus $22 \%$ in the post-Bosman period. Players sourced from Youth academies were least affected in terms of absolute entries, but their relative percentage of entries was reduced by around $11 \%$ as well. It appears that increased competition resulting from the ruling negatively impacted players sourced domestically and in general, clubs were willing to take on greater risks in terms of giving opportunities to unproven players in the Premier League as the total number of new entrants increased significantl .

Table 3.1 breaks down the pre and post Bosman entries by player source in more detail. Looking at the youth program ranking index we can see a shift in hiring patterns for clubs in all categories as a result of the Bosman ruling. The availability 
of foreign talent in the post-Bosman period resulted in a significan shift towards foreign players for all clubs in the study. It appears that experience in foreign leagues outweighs all other factors associated with unproven players in the Premier League. Consistent with the motivating theory, top ranked youth programs (category 1) continued to give the most first-tea opportunities to unexperienced youth players, but this percentage went down from $55 \%$ to $33 \%$ as a result of the Bosman ruling. This percentage went down from $32 \%$ to $23 \%$ for category 2 clubs, and from $47 \%$ to $24 \%$ for category 3 clubs. Domestically sourced players suffered the most in terms of first-tea opportunities, particularly at top ranked youth programs, who substituted away from them and youth players (to a lesser extent) towards foreign players. The hiring patterns changed at the position level as well; Domestically and Youth sourced players were given more opportunities in the defensive positions in the post-Bosman period, rather than in the midfiel and forward positions. It appears that the more technical positions (M and F), where a higher level of ball, dribbling, passing and shooting skills are required, were allocated to players sourced from foreign leagues. This is consistent with certain viewpoints that foreign players are more technically adept than domestic players in the English Premier League. Furthermore, looking at the hiring patterns by club ranking we can see that top ranked clubs (in terms of table standings) shifted the most towards foreign players. When we consider teams ranked lower in the table, their reliance on foreign players increased as well but to a lesser extent than for top ranked clubs; This is particularly true for the lowest ranked club 
category who were fairly consistent in terms of the first-tea opportunities given to players from all three sources. This might signal the much larger resources available to higher ranked clubs that allow them to expand their scouting networks without limits that could be constraining lower ranked clubs. Lower ranked clubs are usually unable to compete for top talent with more reputable clubs, and the process of establishing a scouting network for unproven or unknown foreign talent is very time and resource consuming. Therefore, this suggests that larger clubs were able to shift and/or reallocate their internal resources more quickly and efficientl than lower ranked clubs, as a result of the Bosman ruling.

Focusing on players sourced from youth programs, representing a nation at the junior international level (typically at the U21, U19, or U18 levels) becomes more prevalent for new entrants in the post-Bosman era. In the pre-Bosman period of this study, 45 out 147 youth players made international appearances prior to making a first-tea appearance in the Premier League, while this is the case for 71 out of 149 youth players in the post-Bosman period. Table 3.2 breaks this analysis down further by the youth program ranking of the clubs in this study. Category 1 ranked youth programs maintained their reputation in terms of producing and giving first-tea opportunities to youth internationals. Being an youth-level international became increasingly important to earn first-tea opportunities in lower ranked youth programs as well, which signals an intensifie competitive landscape in the Premier League after the Bosman ruling that filter out top youth talent more efficientl . 


\subsection{Methodology}

The primary objective of this study is to assess whether clubs with more reputable youth programs (in terms of career duration of their graduates) actually provide greater first-tea opportunities to their youth trainees, with a focus on the years surrounding the Bosman ruling. The secondary objective is to examine whether the ranking established by chapter 1 results upholds using this data set, especially in the wake of a major institutional change in the sport. To achieve this, a methodology that builds on a sequence of steps is developed.

\subsubsection{Probit Estimation}

The firs step involves the use of simple probability models to identify factors that are important in providing first-tea opportunities to youth and foreign players, separately. After doing this, the selection problem is modelled as a choice among three possible alternatives. The second step estimates a multinomial logit model structuring the dependent variable as a choice among three possible alternatives. The third and fina step involves the use of survival analysis to examine the career duration patterns of youth trainees in this data set and determine the appropriateness of prior studies in this particular time-frame.

More formally, the task is to estimate a model that characterizes the probabilities of hiring, or specificall giving first-tea opportunities to youth and foreign players. The observation of youth first-tea selection is characterized as a binary 
variable, $Y_{i}$, where:

$$
Y_{i}= \begin{cases}1 & \text { if a youth player is selected. } \\ 0 & \text { otherwise. }\end{cases}
$$

The realization of $Y_{t}$ is used to defin a latent utility measure of youth selection, $Y_{i}^{*}$. Given the available data, this latent utility ${ }^{104}$ can be modelled as:

$$
Y_{i}^{*}=\beta X_{i}+v
$$

where $X_{i}$ represents a set of variables with player characteristics, club information, and certain macro information that might be relevant for youth selection, which is further explained in the subsequent model specification estimated. The error term, $v$, is assumed to be normally distributed with mean zero and constant variance, $\sigma_{v}^{2}$. Under this assumption of normality, the model becomes a probit model and can be estimated using the reduced form:

$$
Y_{t}=\Phi\left(\beta X_{i}\right)+v
$$

where $\Phi\left(\beta X_{i}\right)$ is the cumulative density function (CDF) associated with the normal distribution.

The probability of foreign player selection, $F_{i}$, is modelled in a similar fashion:

$$
F_{i}= \begin{cases}1 & \text { if a foreign player is selected. } \\ 0 & \text { otherwise. }\end{cases}
$$

The realization of $F_{i}$ is used to defin a latent utility measure of the probability of foreign player selection by an English Premier League club, $F_{i}^{*}$. Given the

104 Utility here refer's to a club's utility when selecting new players into the team. 
available data, the following model is employed:

$$
F_{i}^{*}=\alpha D_{i}+u
$$

where $D_{i}$ represents a set of variables with player characteristics, club information, and certain macro information that might be relevant for youth selection, which is further explained in the subsequent model specification estimated. The error term, $u$, is assumed to be normally distributed with mean zero and constant variance, $\sigma_{u}^{2}$. Under this assumption of normality, the model becomes a probit model and can be estimated using the reduced form:

$$
F_{i}=\Phi\left(\alpha D_{i}\right)+u
$$

where $\Phi\left(\alpha D_{i}\right)$ is a CDF associated with the normal distribution.

Three separate probit specification are estimated for youth players being selected in the first-tea by their respective clubs:

- M1: accounts for ranking of player's youth program (Youthranking), share of foreigners in the league (forshare) at the time of entry, player's position, and a constant term.

- M2: M1 plus a dummy variable controlling for entry pre or post the Bosman ruling (Bosman) and the relevant international experience (Inter. Exp.) of the player at the time of entry. 
- M3: essentially M2 with the addition of Manchester United to category 1 youth programs.

Two separate probit specification are estimated for foreign players being given a first-tea opportunity by clubs in the English Premier League during the study period:

- M1: accounts for team ranking (Team ranking) for the club making the first-tea selection, the age of the player at entry (Age), player position, dummy variable controlling for entry pre or post the Bosman ruling (Bosman) and a constant term.

- M2: M1 plus a control for a player's market value at the time of transfer/acquisition (Transfer Value), and his relevant experience at the international level (Inter. Exp.) at the time of entry.

Before moving on to the next stage, the models in (3.1) to (3.5) are tested for specificatio error and fit under the assumption that the two events arise separately. For robustness purposes, the marginal effects of the parameters in estimated models were compared to the ones under a logit representation that assumes a cumulative standard logistic distribution instead; even though the coefficient were different, the marginal effects in the two models were similar as one would expect. The joint significanc of the Youthranking category coefficients as well as the Team ranking 
coefficient in the foreign selection model, was conducted using the common Wald test.

Furthermore, the threat posed by heteroskedastic error terms in probit estimations has been documented well in academia, particularly in Williams (2009). This could be particularly concerning if the basic model is mis-specified While this is a problem that researchers should al least address, there is no consistent remedy that is implemented in practice. Nevertheless, this chapter estimates a maximum-likelihood heteroskedastic probit model a la Harvey (1976), which is a generalization of the probit model that relaxes the assumption of the homoskedastic error term in the probit model. The results indicate that there is no evidence of heteroskedasticity in the error terms in the probit models estimated. More details on the estimation procedure can be found in the appendix.

\subsubsection{Multinomial Logit Estimation}

Up until now, the club's selection problem has been modelled as a binary choice of hiring youths (or foreigners) versus players from all other streams. In fact, there are three possible sources for clubs to utilize when selecting unproven newcomers to the English Premier League; internal (youth stream), and an external stream that can be broken down by players sourced from foreign leagues and players sourced from lower-level domestic leagues. The data accounts for all three sources, and allows for 
estimation where the dependent variable is categorical:

$$
y_{j}=\left\{\begin{array}{l}
1 \text { if } y=j \\
0 \text { if } y \neq j
\end{array}\right.
$$

The multinomial logit model is typically applied in this type of situation, where the probability that club $j$ selects alternative $j$ is:

$$
p_{i j}=p\left(y_{i}=y_{j}\right)=\frac{\exp \left(Z_{i}^{\prime} \gamma_{j}\right)}{\sum_{k=1}^{m} \exp \left(Z_{i}^{\prime} \gamma_{k}\right)},
$$

where $Z_{i}$ represents a set of explanatory variables described below, and $\gamma_{j}$ represents a set of coefficient to be estimated for different alternatives (player sources). One set of coefficient is normalized to zero and coefficient of other alternatives are interpreted in reference to this base outcome, which is represented by domestically sourced players in this chapter. ${ }^{105}$ The inferences from one model would be identical to the other if the baseline comparison category changes. Two multinomial logit specification are estimated and reported:

- M1: Includes categorical variables for youth program ranking (Youthranking), club positional ranking (Team ranking), international experience (Int. Exp.), share of foreigners at time of entry (forshare), and dummy variables for the Bosman ruling (Bosman) and player position.

- M2: M1 with the inclusion of Man $\mathrm{U}$ in the top ranked Youthranking variable.

105 Depending on which alternative is selected as the base category, the estimated coefficient will be different but the marginal effects will be the same regardless of the base category. 
A stringent assumption of multinomial logit models is that outcome categories for the model have the property of independence of irrelevant alternatives (IIA). A general implementation of the Hausman specificatio test is used to test for the validity of IIA, which tests for any systematic differences in the coefficien estimates from the estimated equations. Under the IIA assumption, we would expect no systematic change in the coefficient if we excluded one of the outcomes from the model. Violation of the IIA assumption can lead to inefficien estimates. The parameters are re-estimated by excluding each of the three alternatives, and a Hausman test is performed against the full model. Another aspect worth considering is that the multinomial logit estimates several equations and requires a larger sample size than a binary choice model; given the sample size here, it might be more appropriate to interpret the results from the binary probit estimations. More information on the implementation of the Hausman test can be found in the Appendix.

In addition to the joint significanc test for the Youthranking coefficients a Wald test is performed to test for the equality of these coefficient across the estimated equations.

\subsubsection{Survival Analysis}

In addition to the contents described earlier, the data extends to capture the entire career path for the youth players entering the market during the study period. Most of their observable statistics for each season at the top-tier of major European leagues ${ }^{106}$

106 In addition to the English Premier league, major European leagues are considered to be: Spanish 
are captured, including: appearances, minutes per game, goals, assists (when available), yellow and red cards, international appearances, international minutes per game, each club that a player represents, the club's ranking, each transfer during the career and fee paid for the player, exit from the league and reason for exit, ${ }^{107}$ etc. This type of detail allows for a substantial survival analysis that tests the reliability of chapter 1 results in this much narrower time frame that captures the ever-important Bosman ruling. The duration analysis in this chapter also serves to reinforce the credibility of the youth ranking index. A dummy variable is created to capture the top 5 ranked youth programs from the prior study (category 1 clubs described in the Data section), and analyze their reputation and ability to produce higher quality players in terms of career duration at the top level of European soccer, as compared to lower ranked programs. Consistent with the previous section, a second dummy variable is created that includes Man $U$ in the top-rated youth programs for this particular study period to assess if this alters the results and conclusions in any way.

Wilcoxon and Log-rank tests are performed on the data to test for observed subgroup differences in the survivor functions of players arising from the more reputable versus less reputable youth programs, and the results suggest that there are differences. Figure 3.1 (A) illustrates the subgroup differences in the empirical survival functions, while Figure 3.1 (B) illustrates the same differences in terms of smoothed

La Liga, German Bundesliga, Italian Serie A, and French Ligue 1.

107 Players exiting due to injury are excluded from the sample to generate more reliable estimates that are not impacted by health or related issues. 
empirical hazards. The empirical survivor function (the empirical hazard function) is higher (lower) at all times for youth players originating from more reputable youth academies, ${ }^{108}$ which lends support to previous studies and provides foundations for a more formal modelling approach.

Survival analysis is based on duration models that are used to estimate the hazard rate. The hazard rate, or the instantaneous probability of exit, is estimated using a semiparametric Cox Proportional Hazards (PH) model. ${ }^{109}$ The Cox PH model makes no assumptions about the distribution of survival times and is robust to misspecifica tion of the baseline hazard. A typical semiparametric model is of the form:

$$
h_{i}\left(t_{i} \mid x_{i}, v_{i}\right)=\phi\left(x_{i}\right) \lambda\left(t_{i}\right)
$$

where $\phi\left(x_{i}\right)=\exp \left(x_{i} \beta\right)$ is a function of the observable time-invariant covariates, and $\lambda\left(t_{i}\right)$ is the nonparametric baseline hazard for individual player $i$. The TherneauGrambsch test for the PH assumption, which is based on scaled Schoenfeld residuals, is applied both globally and at specifi covariate level for each specificatio estimated and the results indicate that there is no evidence the $\mathrm{PH}$ assumption is violated. The nonlinearity of covariates is tested for by applying cubic spline functions, and this nonparametric technique does not result in any changes in the assumed linear functional form of the covariates.

108 This difference would be even greater if Man U was included in the more reputable group (Youthrank $=1)$.

109 For a detailed description of possible models that could be employed and all of the statistical tests mentioned in this subsection, please refer to Radoman and Voia (2015). 
The presence of unobserved heterogeneity (frailty) can result in misspecifica tion for several reasons. However, the magnitude of biases in non-frailty models is reduced when we allow for a fully fl xible specificatio for the baseline hazard, like in the Cox model. It is important to note that if the frailty effect is real, the PH model loses its normal proportional hazards property because the hazard ratios are now conditional on the unobserved frailty. Most scholars suggest that interpreting the sign and significanc of the coefficient should be the limit for substantive interpretation of frailty models. Nevertheless, frailty models are estimated using the Gamma distribution for unobserved heterogeneity:

$$
g(v)=\frac{v^{\frac{1}{\theta}-1} \exp \left(\frac{-v}{\theta}\right)}{\Gamma\left(\frac{1}{\theta}\right) \theta^{\frac{1}{\theta}}}
$$

Three different specification are estimated using the Cox model:

- M1: Includes a dummy variable for more reputable youth programs (without Man U), performance measures (appearances (Apps), international appearances (Intapps), average minutes played per game (Mpg), goals scored (Goals), yellow cards obtained (Yellow), per season), dummy control for entering pre or post the Bosman ruling (Bosman), and position played by the player.

- M2: M1 with the inclusion of Man $\mathrm{U}$ in the more reputable youth program category. 
- M3: M2, additionally accounting for unobserved heterogeneity.

\subsection{Independent Probit Results}

Separate probit estimation is conducted for the probability of selection of players sourced internally (youth) and from foreign leagues. The main assumption of independent estimation is that the error terms in the two models are not correlated. This notion will be tested in the next section, where these independent specification are nested in a bivariate probit model that accounts for correlation in the unobservables.

\subsubsection{Youth Selection}

Table 3.3 presents the results of three probit specification for youth selection. Looking at the log likelihood for each specification there is a significan improvement when moving from M1 to either M2 or M3. Consequently, the AIC and BIC model fi statistics show a significan improvement when moving from M1 to M2/M3. ${ }^{110}$ Controlling for the Bosman ruling and player experience at the international level improves the model specificatio substantially. Including Man U in the category 1 youth programs (M3) improves the log likelihood only slightly from M2, but the significanc of the youth program ranking coefficient is significantl improved to the point that all categories are significan at the $1 \%$ level.

110 The AIC for M1 is 1098, 867 for M2 and 863 for M3. Similarly, BIC for M1 is 24, -194 for M2 and -198 for M3. Both of these criteria suggest that lower values correspond to better model fit 
The results support the theoretical intuition that youth players arising from better ranked youth programs have a higher probability of being selected in the first team of their parent club. Panel A of table 3.6 provides the predicted probabilities associated with arising from the four differently ranked youth programs from M3; the results indicate that category 1 clubs have a $14-15 \%$ higher probability of selecting a youth player in their first-tea than lower ranked youth programs. These clubs invest more heavily in their youth academies and could be assumed to be more "bottom-heavy", so players arising from their youth programs have a higher probability of being promoted to the first-team The results lend support to the theory that more "bottom-heavy", or youth oriented in this case, clubs provide greater opportunities for higher-level positions (first-team to employees from internal sources. The unique youth ranking index created in this chapter measures the degree of "bottomheaviness" by Premier League clubs, and the estimated results are aligned with the index ranking of clubs in terms of opportunities provided to internally sourced players. What could be just as important is that the youth training reputation of these clubs is consistent in the wake of a major institutional change, the Bosman ruling, that presented clubs in Europe with more options in their talent search and strategic/optimization decisions. Even though the overall first-tea opportunities to youth players became scarcer in all clubs, the higher ranked youth programs continued to provide more opportunities to internally sourced players relative to lower ranked 
clubs. Similar results are obtained from M2, except that category 2 youth programs do not have a significan coefficien at a reasonable level of significance

Looking at the player's position effect on selection, the benchmarking was based on the goaltender position but that position's representation in the data was by far the smallest so one has to be careful with the interpretation of these results. There is only one available slot on the first-tea for a goaltender versus other positions that have multiple slots available for rotation, and goaltenders tend to be replaced less often than the other positions. The results indicate that youth players are most often selected in the midfiel and defensive positions and least often in the forward position, even though the coefficien for forwards is not significan at any reasonable level. This suggests that foreign (or other) sources are used more often for the most scrutinized and demanding position in European soccer, the forward position. The effect of the share of foreign players in the league (forshare) changes signs when moving from M1 to M2 or M3, and it increases in significanc as well. Controlling for the Bosman ruling alters the sign of this covariate and results indicate there is a positive effect on youth selection with increases in the share of foreigners in the league. The coefficien for the Bosman ruling was negative and significan at the $1 \%$ level, as anticipated. This indicates, in general, that youth players have a lower probability of being selected after the ruling, which is not surprising considering the talent pool available for clubs to draw from increased significantl as a result of this institutional change. However, the more important result here is that the more reputable 
youth clubs provided more opportunities through internal promotion even in the presence of this external shock. There is a significan effect of international experience on youth player selection as well. The negative correlation between international experience at the senior level and youth selection is not surprising because youth player do not have this sort of experience typically at their tender age. However, and more importantly, international experience at the junior levels has a positive and statistically significan effect (at the $1 \%$ level) on youth selection that is not too different in M2 and M3. This indicates the youth players with international experience at junior levels provide a strong signal to their employers about their potential and future inclusion in the first-team

Tables 3.4 and 3.5 outline the tests performed on the appropriateness of model specificatio and a measure of fit In table $3.4 \mathrm{fi}$ is measured by comparing the mean and standard deviation of actual youth selection against the corresponding moments implied by the estimated probit model. The results indicate a close match between the two distributions, with the means being almost identical. Table 3.5 provides the test of misspecificatio error for the estimated model. The joint significanc test for the Youthranking coefficient indicates they are statistically different from zero. The test regresses the link function of the outcome variable (the probability function) on the predicted probability and the predicted probability squared. The intuition is that the predicted probability function should be statistically significan unless the model is mis-specified Proper specificatio also implies that the squared probability should 
not have predictive power. The associated link test supports the specificatio of the model at the $1 \%$ level of significance while indicating that the squared prediction is not significan at any reasonable level. ${ }^{111}$ There is no indication that the model is mis-specified

\subsubsection{Foreign Selection}

Table 3.7 presents the results of two probit specification for foreign source selection of players. In terms of log likelihood, there is a significan improvement when moving to M2 from M1. Correspondingly, the AIC and BIC statistics are both much lower for M2, 648 and -442 respectively, than for M1, 836 and -267, respectively. Model specificatio is improved when controlling for additional factors, including a player's market (transfer) value and his experience at the international level at the time of entry.

The results indicate that a club's ranking/grouping in the table significantl affects the probability of a foreign player being selected in the first-team The ordering of the team ranking coefficient suggests that higher ranked clubs, or those fight ing for the league title or a place in European competitions, have a tendency to hire and play more foreigners in their first-tea than lower-ranked clubs. Panel B of table 3.6 outlines the predicted probabilities for the team ranking covariate; clubs ranked in the firs tier have an $11 \%$ greater probability of selecting players from foreign sources

111 M2 and M3 results indicate that the model is not misspecifie at the $1 \%$ level of significance However, M1's results are indicative only at the $10 \%$ level, which further outlines the improvement in specificatio when controlling for additional covariates. 
than clubs ranked in the second tier, and $15 \%$ greater probability than clubs ranked in the third and bottom tier. Higher ranked clubs are typically financiall superior, and this indicates that their financia strength allows them to rely on external sources (i.e. the transfer market) for new additions to the first-team This could be an indication that lower-ranked clubs, which can be assumed to be less well-off financiall, cannot compete for talent in the transfer market with larger clubs, even after the Bosman ruling. One would assume that larger clubs had more of an advantage in terms of securing talent from external sources prior to the Bosman ruling when the quota for foreign players restricted the amount of spots for them in the first-team However, it appears that the removal of the quota did not close the gap among these differently ranked clubs in terms of their ability to scout abroad. In fact, the gap grew even further in favor of larger clubs signalling their ability to adapt to the new environment more efficientl .

The age of the foreign sourced player has a positive and statistically significan (at the $1 \%$ level) effect on selection in the firs team. This suggests that experience in foreign leagues is valued more than raw potential of youth by Premier League clubs. Interestingly, the position dummies lose significanc when we move from M1 to M3. M2 results indicate that foreign players are recruited more at the most technical positions, midfiel and forwards, which is consistent with the patters described in the data section of this chapter. Players are selected from foreign sources at the forward position with greatest probability. However, as we move to a better specifie model 
(M3), the loss in significanc of these position coefficient renders them irrelevant for interpretation purposes. The Bosman ruling has a strong and predictable positive effect on foreign player selection, meaning that their probability of selection by one of the Premier League clubs increased significantl after the ruling. The positive effect does diminish as we improve model specificatio in M3. In addition, a foreign player's market (transfer) value has a positive and statistically significan effect on his first-tea selection; the higher the player's transfer value, the higher the probability he gets an opportunity in the first-team A player's senior international experience for his country signals his quality and has a predictable positive effect on his selection, statistically significan at the $1 \%$ level.

The goodness of $\mathrm{ft}$ and misspecificatio tests are conducted in the same fashion as for selection of youth players, but the resulting tables are not reported. There is a close match between the mean and standard deviation of actual foreign player selection against the corresponding moments implied by the estimated probit model, which indicates a good fi of the model. In addition, the link test for both specifi cations indicates that the models are not mis-specifie at the $1 \%$ level of statistical significance The joint significanc test for the Team ranking coefficient indicates that they are statistically different from zero. 


\subsection{Multinomial Logit Results}

In this type of model, we have an unordered categorical dependent variable. The basic idea is that the decision by clubs to hire new and unproven Premier League players from internal or external sources encompasses three possible choices: (i) players from their own youth system, (ii) players sourced from lower leagues, and (iii) players sourced from lower-level domestic leagues. The independent probit modelled youth or foreign selection as a binary choice between internal promotion and external hiring, while the multinomial logit breaks down the externally sourced players in two separate groups for a total of three alternatives available to clubs. The results are structured and interpreted against the base alternative, which consists of domestically sourced players from lower-level leagues.

Table 3.8 outlines the estimation results for youth and foreign sources vs. the base domestic source, and the interpretation here relates to the top panel of the data, which represents the intended segment of the research at hand. The youth program ranking is highly statistically significan for all categories in the index for players sourced internally (Youth), with category 2 being significan at the $5 \%$ level and all others at the $1 \%$ level. The negative coefficient indicate that the relative log odds of youth selection vs. domestically sourced players decreases by 1.20 if the youth player originates from a category 4 vs. category 1 youth training program. Once again, this result reiterates the independent probit results that the probability of youth selection is higher at higher ranked youth clubs. Panel $\mathrm{C}$ of Table 3.6 provides the predicted 
probabilities of youth selection for clubs in all four youth ranking categories for M2; youth players originating from category 1 youth programs have a $31 \%$ probability of being selected in the first-team while the next highest category has a $22 \%$ probability of youth selection. These results are consistent with the independent probit results for youth selection, and similar reasoning is applicable relevant to the motivating theory. The player position dummies are statistically significan for both specifications indicating that youth players have the highest probability of being selected in midfiel position vs domestically sourced players. Representing a country in the junior ranks has a predictable and statistically significan effect on the probability of youth selection, as it provides a strong signal about a player's ability and potential. Unsurprsingly, the Bosman dummy is negative and statistically significan (at the $1 \%$ level) implying the ruling had a negative effect on youth selection.

In terms of log-likelihood, there isn't much difference in the two specification indicating that the results are robust and interpretation is similar whether Man $\mathrm{U}$ is included in category 3 or 1 . Both specification fi the data well in terms of improvement in log-likelihood from a baseline or constant only model. The improvements in the significanc are also evident in the AIC and BIC criteria when we move towards the M1 and M2 specification from the baseline model. Joint significanc of the Youthrank coefficient in all equations indicates the overall effect of the youth ranking index is statistically significan at the $1 \%$ level. In addition, there is no ev- 
idence that the coefficient in the estimated equations are different. ${ }^{12}$ The marginal effects of the youth ranking index categories are all statistically significan and consistent with the ranking implied by the index for M2. This is particularly important because much like the estimated probabilities listed in Table 6, these effects do not vary with the choice of the base category for comparisons, unlike the displayed coefficient (log-rank ratios) that differ for each chosen base category.

Considering the goodness of fit there is a close match between the firs and second moments from the actual selections and the ones implied by the estimated multinomial logit model. ${ }^{113}$ Upon examining the output from the Hausman test, there is no evidence that the IIA assumption has been violated in any of the scenarios tested.

\subsection{Survival Analysis Results}

The results of the semiparametric estimations are presented in Table 3.9. There isn't much difference in $\log$ likelihoods of M1 and M2, but the frailty specificatio (M3) significantl improves the likelihood of the model. The evidence of unobserved heterogeneity is statistically significant but only at the $10 \%$ level. The estimated parameter $\theta$, a measure of heterogeneity or overdispersion, is 0.06 . Typically, a lower measure of heterogeneity is preferred, and the lower it is the more reliable the inter-

112 This is true for all of the estimated coefficients as well as for the youth ranking index tested independently.

113 The results are similar to Table 4 results, and are not presented in the paper. 
pretation of the estimated hazard ratios becomes. Therefore, the rather low value of the overdispersion and its statistical signific nce allow for a more reasonable interpretation of the results. Nonetheless, the results will be interpreted in terms of M1 and $\mathrm{M} 2$, but the general conclusions are consistent among all specifications

Players originating from a more reputable youth program have an exit probability that is $19 \%(26 \%)$ lower in M1 (M2) than players from the rest of the youth programs considered here, which is statistically significan in all specifications This result validates the empirical observations and the benchmark results from chapter 1. Youth program participation and training has an impact on a player's career path, and duration at the top-level of European soccer. The performance measures have a statistically significan and predictable negative effect on the hazard rate in all specification each additional appearance per season reduces the exit probability for a player by $4 \%$, an additional 10 minutes spent on the pitch per game decreases the exit probability by $6-7 \%$, each international appearance reduces the exit probability by $16-17 \%$, and each goal scored decreases the exit probability by $13 \%$. The disciplinary measure, yellow cards, presents an interesting and statistically significan result, which indicates that an additional yellow card per season increases the exit probability by $7 \%$. This means that more disciplined players will tend to have longer careers as this is a valued player virtue among Premier League clubs, which is that much more important for youth players in the tender and early years of their careers. The Bosman ruling dummy is not significan at any reasonable level. Out of the four 
positions considered, it is not surprising that goaltenders tend to have the longest careers. The dummies for defenders and midfielder are significan (at the 5\% and 10\% level, depending on the model) and suggest that defenders have a lower exit probability than midfielders while the forward dummy is not significant This suggests that youth players occupying positions that require lower technical skills have longer careers at the top level.

The results indicate that unobserved heterogeneity is not statistically significan at the player level, but it is significan at the youth program (team) level, which is presented in M3. This suggests that there is something unique to each youth program, rather than at a player talent level. At least it can be said that youth sourced players in this sample, who made at least one appearance in the Premier League, appear to be fairly homogenous, while their youth-club training generates heterogeneity among them that could be a deciding factor in their careers.

Figure 3.2 (A) displays the baseline hazards of all three specifications which show that duration dependence is modelled adequately by all models in terms of mimicking the slope of the empirical hazard. Figure 3.2 (B) shows the estimated hazards for M2 and M3, with M1 omitted due to its nearly identical alignment with M2. Both specification slightly underpredict the empirical hazard and are fairly close to each other, with M2 being marginally higher and closer to the empirical hazard. In addition to the visual techniques, Cox-Snell residuals are used to assess 
the goodness of fi for M1 and M2, which provided evidence of satisfactory fi of the data. $^{114}$

\subsection{Conclusion}

This chapter establishes a connection between relevant labor theory and the hiring/promotion decisions of English Premier League clubs, when it comes to offering first-tea opportunities to new and unproven players. Two proprietary data sets are combined to address the research questions at hand, using a sequential econometric approach that addresses multiple issues. More reputable clubs in terms of producing higher-quality youth players display a higher degree of "bottom-heaviness", and as such provide more opportunities for their youth players through internal promotion. These results are consistent in the wake of the Bosman case ruling, which certainly had an effect on clubs' decision making process. Unsurprisingly, foreign sourced players increased their presence in the league significantl after the removal of the foreign player quota, and became the dominant stream for acquisition of new players. Better ranked, or financiall superior, clubs had a higher probability of selecting foreign players, which speaks to their ability to re-allocate their internal resources more efficientl towards foreign-player scouting than smaller (lower-ranked) clubs when accounting for the Bosman ruling.

114 For specifi detail on the tests mentioned in this section, please refer to Radoman and Voia (2015). 
In addition to the main results and objectives, the survival analysis in this chapter upholds the results established in chapter 1 and demonstrates that the best-ranked youth programs consistently outperform the others in terms of career duration of their youth products in this narrower sample that controls for the Bosman ruling. Unobserved heterogeneity is statistically significan at the parent youth-club level, rather than at the player level, which lends further support to the notion that each youth academy has differential ability in "schooling" their players at the youth level that plays an important role in the careers of otherwise homogeneous players.

One of the limitations of this data is that it does not account for all of the youth players under contract in the clubs examined. This sort of detail, which could become possible and accessible in future years with improvements in data availability, would aid in providing a more reasonable assessment of "bottom-heaviness" of clubs and better address their probability of promoting from within. Such an analysis can be extended to other sports that are structured in a similar fashion, like European basketball or hockey in North America. The internal vs. external hiring decisions and associated labor economics theories have significan potential to be empirically tested further in the sports economy sector. 


\subsection{Appendix}

\subsubsection{Heteroskedastic Probit Model}

Heteroskedastic probit model is a generalization of the ordinary probit model. It generalizes the model by allowing the variance to differ from 1 , and vary as a function of the independent variables in a normal CDF $\Phi$. The variance is assumed to be a multiplicative function of these $m$ independent variables $\mathbf{x}_{i}=\left(x_{1 i}, x_{2 i}, \ldots, x_{m i}\right)$, as in Harvey (1976):

$$
\sigma_{i}^{2}=\left\{\exp \left(x_{i} \gamma\right)\right\}^{2}
$$

Therefore, the estimation of the probability of success becomes:

$$
\operatorname{Pr}\left(y_{i}=1\right)=\Phi\left\{\frac{z_{i} b}{\exp \left(x_{i} \gamma\right)}\right\}
$$

where no constant term can be present in $x_{i} \gamma$, unlike in $z_{i} b$ (linear combination of the independent variables, known as the index function) for the model to be identifiable Relaxing the homoskedastic assumption of the probit model in this manner yields the multiplicative heteroskedastic probit model. This model is difficul to fit and often requires many iterations. The likelihood-ratio test of heteroskedasticity tests the full model with heteroskedasticity against the full model without.

The M3 Youth selection probit model was estimated in this fashion, and all of the covariate coefficient were not statistically significan at the $10 \%$ level. The reported likelihood-ratio $\chi^{2}(10)$ test statistic was $13.57,{ }^{115}$ which is not statistically sig-

115 The estimated likelihood-ratio test statistic for the M2 specificatio (without Man U in category 1) 
nifican at any reasonable level. Therefore, there was no indication that heteroskedasticity is present in the standard probit estimation of the model. Similarly, the reported likelihood-ratio test for the M2 Foreign selection heteroskedastic model was 11.10, which is also not statistically significan at any reasonable level.

\subsubsection{Note on the Hausman Test of IIA}

The Hausman test is a standardized comparison of model coefficients so using it requires that the base outcome be the same in both competing models. The multinomial logit model was estimated three separate times, one as presented in the results ( $m 1)$ and the other two by removing one of the other categories from the model $(m 2$ by removing the youth category and $m 3$ by removing the foreign player category). The direct comparison of $m 1$ and $m 3$ coefficient under the Hausman test returned a $\chi^{2}(13)$ test statistic of 17.33 , which is not statistically significan at any reasonable level indicating that we can not conclude the difference in coefficient is not systematic, upholding the IIA assumption. However, when comparing $m 1$ and $m 2$, the $\chi^{2}(13)$ test statistic is negative $(-1.76)$, which suggests model fitte on this data does not satisfy the asymptotic assumptions of the Hausman test. This kind of result is not uncommon for the Hausman test, particularly in smaller sample sizes; it indicates the Hausman was not well defined The problem is due to the estimator of the variance, which is a feasible estimator only asymptotically.

was 14.74 , which was also not statistically significan at any reasonable level. 
There is an alternative to the Hausman test that overcomes this problem through an alternative estimator of the variance of the difference between the two estimators, using the seemingly unrelated estimation test. This other estimator is guaranteed to be positive semidefinite This alternative estimator also allows a widening of the scope of problems to which Hausman-type tests can be applied by relaxing the assumption that one of the estimators is efficient The estimation of the simultaneous covariance of a series of $k$ estimators is a nonstandard application of the sandwich estimator. This estimator for the covariance of estimators is an application of the cluster modificatio of the sandwich estimator proposed by Rogers (1993). The sandwich estimator of the covariance $V_{12}$ is compared with the estimator of variance $\tilde{V}_{12}$ using the classic Hausman test. Although the earlier Hausman test computed for $m 3$ and $m 1$ was not defined the seemingly unrelated estimation test cannot conclude there are any systematic patters in the estimated coefficient in the two equations. 


\subsection{Tables and Figures}

Table 3.1: Breakdown by Source

\begin{tabular}{c|ccc|ccc}
\hline \hline Variables & \multicolumn{3}{|c|}{ Pre-Bosman } & \multicolumn{3}{c}{ Post-Bosman } \\
\hline Ythrank & Domestic & Youth & Foreign & Domestic & Youth & Foreign \\
\cline { 2 - 7 } 1 & 16 & 40 & 17 & 9 & 35 & 63 \\
2 & 14 & 15 & 18 & 13 & 22 & 60 \\
3 & 28 & 44 & 22 & 28 & 26 & 55 \\
4 & 73 & 48 & 22 & 60 & 66 & 63 \\
\hline Position & & & & & & \\
G & 11 & 6 & 4 & 13 & 7 & 19 \\
D & 48 & 45 & 24 & 44 & 56 & 73 \\
M & 34 & 66 & 28 & 32 & 49 & 75 \\
F & 38 & 30 & 23 & 21 & 37 & 74 \\
\hline Clubrank & & & & & & \\
Top 7 & 32 & 34 & 24 & 15 & 43 & 89 \\
$8-15$ & 42 & 62 & 26 & 47 & 56 & 91 \\
Bottom 5 & 57 & 51 & 29 & 48 & 50 & 61 \\
\hline
\end{tabular}

Table 3.2: Youth Breakdown by International Experience

\begin{tabular}{c|cc|cc}
\hline \hline \multicolumn{2}{c}{} & Pre-Bosman & \multicolumn{2}{c}{ Post-Bosman } \\
\hline Ythrank & Yes & No & Yes & No \\
1 & 20 & 20 & 18 & 17 \\
2 & 4 & 11 & 9 & 13 \\
3 & 10 & 34 & 11 & 15 \\
4 & 11 & 37 & 33 & 33 \\
\hline
\end{tabular}


Table 3.3: Probit Results for Youth Selection

\begin{tabular}{|c|c|c|c|}
\hline & M1 & M2 & M3 \\
\hline \multicolumn{4}{|c|}{ Youthranking - 1 is the benchmark } \\
\hline 2 & $\begin{array}{c}-.42^{* *} \\
(.15)\end{array}$ & $\begin{array}{c}-.37^{* *} \\
(.17)\end{array}$ & $\begin{array}{c}-.42^{* * * *} \\
(.17)\end{array}$ \\
\hline 3 & $\begin{array}{l}-.22 \\
(.13)\end{array}$ & $\begin{array}{l}-.25 \\
(.15)\end{array}$ & $\begin{array}{c}-.41^{* * *} \\
(.16)\end{array}$ \\
\hline 4 & $\begin{array}{c}-.19^{*} \\
(.12)\end{array}$ & $\begin{array}{c}-.37^{* * *} \\
(.14)\end{array}$ & $\begin{array}{c}-.42^{* * *} \\
(.13)\end{array}$ \\
\hline forshare & $\begin{array}{c}-.009^{*} \\
(.005)\end{array}$ & $\begin{array}{c}.05^{* * *} \\
(.01)\end{array}$ & $\begin{array}{c}.05^{* * *} \\
(.01)\end{array}$ \\
\hline \multicolumn{4}{|c|}{ Position - Goalie is the benchmark } \\
\hline defence & $\begin{array}{c}.42^{* *} \\
(.20)\end{array}$ & $\begin{array}{c}.45^{* *} \\
(.22)\end{array}$ & $\begin{array}{c}.45^{* *} \\
(.22)\end{array}$ \\
\hline midfiel & $\begin{array}{c}.54^{* * *} \\
(.20)\end{array}$ & $\begin{array}{c}.59^{* * *} \\
(.22)\end{array}$ & $\begin{array}{c}.60^{* * *} \\
(.22)\end{array}$ \\
\hline forward & $\begin{array}{l}.25 \\
(.20)\end{array}$ & $\begin{array}{l}.33 \\
(.23)\end{array}$ & $\begin{array}{l}.34 \\
(.23)\end{array}$ \\
\hline Bosman & & $\begin{array}{c}-.94^{* * *} \\
(.28)\end{array}$ & $\begin{array}{c}-.95^{* * *} \\
(.28)\end{array}$ \\
\hline \multicolumn{4}{|c|}{ Inter. Exp. - no exp. is the benchmark } \\
\hline 1 (Senior) & & $\begin{array}{c}-2.26^{* * *} \\
(.27)\end{array}$ & $\begin{array}{c}-2.27^{* * *} \\
(.27)\end{array}$ \\
\hline 2 (Junior) & & $\begin{array}{c}.31^{* * *} \\
(.11)\end{array}$ & $\begin{array}{c}.30^{* * *} \\
(.11)\end{array}$ \\
\hline Const. & $\begin{array}{l}-.23 \\
(.29)\end{array}$ & $\begin{array}{c}-1.83^{* * *} \\
(.52)\end{array}$ & $\begin{array}{c}-1.79^{* * *} \\
(.52)\end{array}$ \\
\hline Observations & 857 & 857 & 857 \\
\hline Log Likelihood & -540.18 & -420.91 & -418.74 \\
\hline
\end{tabular}

Table 3.4: Goodness of Fit

\begin{tabular}{c|cc|cc}
\hline \hline Variable & Mean (M3) & St. Dev. (M3) & Mean (M2) & St. Dev. (M2) \\
\hline Youth Selection (actual) & .3442 & .4754 & .3442 & .4754 \\
Fitted prob. of youth selection & .3448 & .2346 & .3447 & .2322 \\
\hline \hline
\end{tabular}

Table 3.5: Link Test for Misspecificatio - Youth

\begin{tabular}{ccc}
\hline \hline Youth Selection & Coefficien (M3) & Coefficien (M2) \\
\hline Prediction & $1.11^{* * *}$ & $1.11^{* * *}$ \\
& $(.16)$ & $(.17)$ \\
Prediction squared & .07 & .06 \\
& $(.08)$ & $(.08)$ \\
\hline \hline
\end{tabular}

Note: $* * *$ indicates statistical significanc at 0.01 level 
Table 3.6: Predicted Probability Analysis

\begin{tabular}{|c|c|c|c|c|c|}
\hline \multicolumn{2}{|l|}{ Youth (A) } & \multicolumn{2}{|l|}{ Foreign (B) } & \multicolumn{2}{|c|}{ "Multinomial (C) } \\
\hline Ythrank & Margin (M3) & Team rank & Margin (M2) & Ythrank & Margin (M2) \\
\hline 1 & $\begin{array}{l}.34^{* * *} \\
(.04)\end{array}$ & 1 & $\begin{array}{c}.45^{* * *} \\
(.04)\end{array}$ & 1 & $\begin{array}{l}.31^{* * *} \\
(.05)\end{array}$ \\
\hline 2 & $\begin{array}{c}.20^{* * *} \\
(.04)\end{array}$ & 2 & $\begin{array}{c}.34^{* * *} \\
(.04)\end{array}$ & 2 & $\begin{array}{c}.18^{* * *} \\
(.04)\end{array}$ \\
\hline 3 & $\begin{array}{c}.20^{* * *} \\
(.04)\end{array}$ & 3 & $\begin{array}{c}.30^{* * *} \\
(.03)\end{array}$ & 3 & $\begin{array}{l}.22^{* * *} \\
(.05)\end{array}$ \\
\hline 4 & $\begin{array}{c}.19^{* * *} \\
(.03)\end{array}$ & & & 4 & $\begin{array}{l}.21^{* * *} \\
(.04)\end{array}$ \\
\hline
\end{tabular}

Note: $* * * \overline{\text { indicates statistical significanc at } 0.01 \text { level }}$

Table 3.7: Probit Results for Foreign Selection

\begin{tabular}{lcc}
\hline \hline & $\mathrm{M} 1$ & $\mathrm{M} 2$ \\
\hline Team ranking - 1 is the benchmark & $-.36^{* * *}$ & $-.28^{* *}$ \\
2 & $(.13)$ & $(.15)$ \\
3 & $-.59^{* * *}$ & $-.39^{* * *}$ \\
& $(.13)$ & $(.15)$ \\
Age & $.20^{* * *}$ & $.14^{* * *}$ \\
& $(.01)$ & $(.02)$ \\
Position - Goalie is the benchmark & & -.03 \\
def & .20 & $(.22)$ \\
& $(.21)$ & .14 \\
mid & $.44^{* *}$ & $(.22)$ \\
& $(.21)$ & .25 \\
for & $.60^{* * *}$ & $(.23)$ \\
Bosman & $(.21)$ & $.35^{* * *}$ \\
& $.64^{* * *}$ & $(.12)$ \\
Transfer Value & $(.10)$ & $2.96 \mathrm{e}-07^{* * *}$ \\
& & $(5.53 \mathrm{e}-08)$ \\
Inter. Exp. - no exp. is the benchmark & & \\
1 (Senior) & & $1.42^{* * *}$ \\
& & $(.15)$ \\
(Junior) & & .17 \\
Const. & -409.01 & $(.14)$ \\
& & $-4.33^{* * *}$ \\
Obs. & & $(.48)$ \\
\hline \hline **, and *** indicates statistical significanc at the $0.1,0.05$, and 0.01 levels, respectively. & 857 \\
& & -311.45 \\
\hline
\end{tabular}


Table 3.8: Multinomial Logit Results

\begin{tabular}{|c|c|c|}
\hline & M1 & M2 \\
\hline \multicolumn{3}{|c|}{ Base - Domestic Source } \\
\hline \multicolumn{3}{|c|}{ Youth Source } \\
\hline \multicolumn{3}{|c|}{ Youthranking - 1 is the benchmark } \\
\hline 2 & $-.79^{* *}(.36)$ & $-.87^{* *}(.34)$ \\
\hline 3 & $-.83^{* * *}(.31)$ & $-1.12^{* * *}(.31)$ \\
\hline 4 & $-1.20^{* * *}(.30)$ & $-1.33^{* * *}(.29)$ \\
\hline \multicolumn{3}{|c|}{ Team ranking - 1 is the benchmark } \\
\hline 2 & $-.18(.25)$ & $-.05(.25)$ \\
\hline 3 & $-.17(.27)$ & $-.02(.27)$ \\
\hline \multicolumn{3}{|c|}{ Inter. exp. - no exp. is the benchmark } \\
\hline 1 (senior) & $-2.86^{* * *}(.74)$ & $-2.84 * * *(.74)$ \\
\hline 2 (junior) & $.55^{* * *}(.22)$ & $.52^{* *}(.22)$ \\
\hline \multicolumn{3}{|c|}{ Position - Goalie is the benchmark } \\
\hline $\mathrm{D}$ & $.71^{*}(.39)$ & $.73^{*}(.50)$ \\
\hline M & $1.12^{* * *}(.40)$ & $1.14^{* * *}(.40)$ \\
\hline $\mathrm{F}$ & $.72 *(.41)$ & $.74^{*}(.41)$ \\
\hline forshare & $.11^{* * *}(.03)$ & $.11^{* * *}(.03)$ \\
\hline Bosman & $-1.69^{* * *}(.50)$ & $-1.71^{* * *}(.50)$ \\
\hline constant & $-3.40^{* * *}(.95)$ & $-3.44^{* * *}(.94)$ \\
\hline \multicolumn{3}{|c|}{$\begin{array}{l}\text { Foreign Source } \\
\text { Youthranking - } 1 \text { is the benchmark }\end{array}$} \\
\hline 2 & $-.26(.37)$ & $-.23(.36)$ \\
\hline 3 & $-.99^{* * *}(.35)$ & $-1.05^{* * *}(.35)$ \\
\hline 4 & $-1.47^{* * *}(.33)$ & $-1.47^{* * *}(.33)$ \\
\hline \multicolumn{3}{|c|}{ Team ranking - 1 is the benchmark } \\
\hline 2 & $-.42(.27)$ & $-.30(.28)$ \\
\hline 3 & $-.22(.30)$ & $-.11(.31)$ \\
\hline \multicolumn{3}{|c|}{ Inter. exp. - no exp. is the benchmark } \\
\hline 1 (senior) & $2.46^{* * *}(.26)$ & $2.47^{* * *}(.26)$ \\
\hline 2 (junior) & $.13(.27)$ & $.12(.27)$ \\
\hline \multicolumn{3}{|c|}{ Position - Goalie is the benchmark } \\
\hline $\mathrm{D}$ & $.02(.40)$ & $.03(.40)$ \\
\hline M & $.40(.41)$ & $.41(.41)$ \\
\hline $\mathrm{F}$ & $.41(.41)$ & $.42(.41)$ \\
\hline forshare & $.08^{* * *}(.03)$ & $.08^{* * *}(.03)$ \\
\hline Bosman & $-.22(.48)$ & $-.25(.48)$ \\
\hline constant & $-2.84(.97)$ & $-2.97(.97)$ \\
\hline Log Likelihood & -680 & -677 \\
\hline
\end{tabular}


Table 3.9: Cox Semiparametric Survival Results

\begin{tabular}{lccc}
\hline \hline & $\mathrm{M} 1$ & $\mathrm{M} 2$ & $\mathrm{M} 3$ \\
\hline Youthrank & $.81^{* *}$ & $.74^{* * *}$ & $.63^{* *}$ \\
& $(.07)$ & $(.06)$ & $(.11)$ \\
Apps & $.96^{* * *}$ & $.96^{* * *}$ & $.96^{* * *}$ \\
& $(.007)$ & $(.007)$ & $(.01)$ \\
Intapps & $.83^{* * *}$ & $.84^{* *}$ & $.71^{* *}$ \\
& $(.06)$ & $.06)$ & $(.12)$ \\
Mpg & $.994^{* * *}$ & $.993^{* * *}$ & $.993^{* *}$ \\
& $(.002)$ & $(.002)$ & $(.003)$ \\
Goals & $.87^{* * *}$ & $.87^{* * *}$ & $.88^{* *}$ \\
& $(.04)$ & $(.03)$ & $(.06)$ \\
Yellow & $1.07^{* *}$ & $1.07^{* *}$ & $1.09^{*}$ \\
& $(.03)$ & $(.03)$ & $(.06)$ \\
Bosman & 1.04 & 1.03 & 1.11 \\
& $(.08)$ & $(.08)$ & $(.13)$ \\
Player Position - Goalie $i s$ the benchmark & & \\
Defence & $.66^{*}$ & $.68^{*}$ & $.53^{* *}$ \\
& $(.14)$ & $(.15)$ & $(.16)$ \\
Midfiel & $.64^{* *}$ & $.66^{*}$ & $.44^{* * *}$ \\
& $(.14)$ & $(.15)$ & $(.14)$ \\
Forward & .81 & .82 & $.56^{*}$ \\
& $(.18)$ & $(.19)$ & $(.19)$ \\
\hline$\theta$ & & & $.06^{*}$ \\
& & 1537 & $(.05)$ \\
Obs. & 1537 & -1941 & 938 \\
Log Likelihood & -1944 & -1349 \\
\hline \hline Note: ${ }^{* * *}$, and ${ }^{* * *}$ indicates statistical significanc at the $0.1,0.05$, and 0.01 levels, respectively. &
\end{tabular}

Note: ${ }^{*}, * *$, and ${ }^{* * *}$ indicates statistical significanc at the $0.1,0.05$, and 0.01 levels, respectively. 

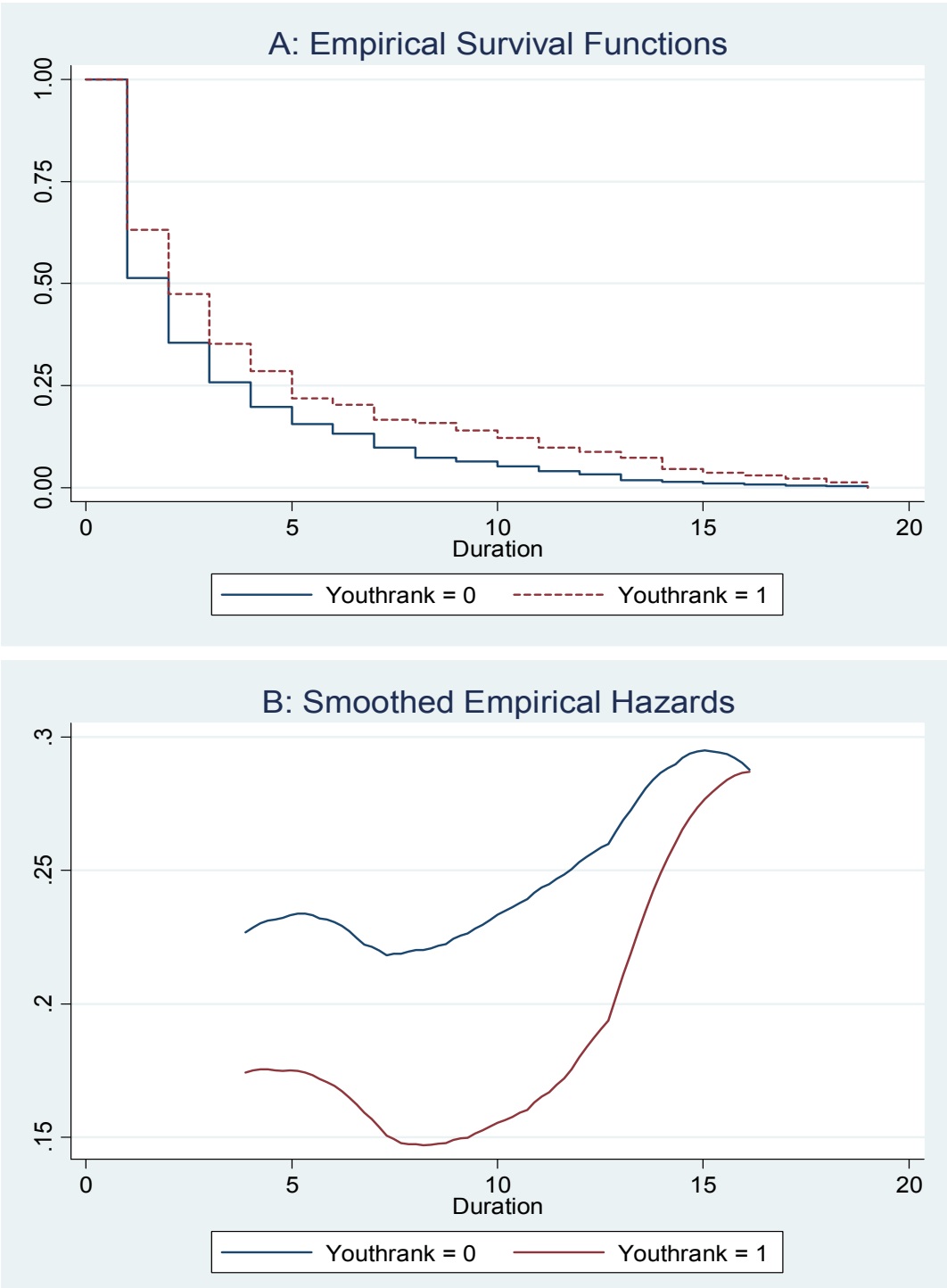

Figure 3.1: (A) Empirical Survivor Functions by Youthteam, (B) Empirical Hazard Functions by Youthteam 

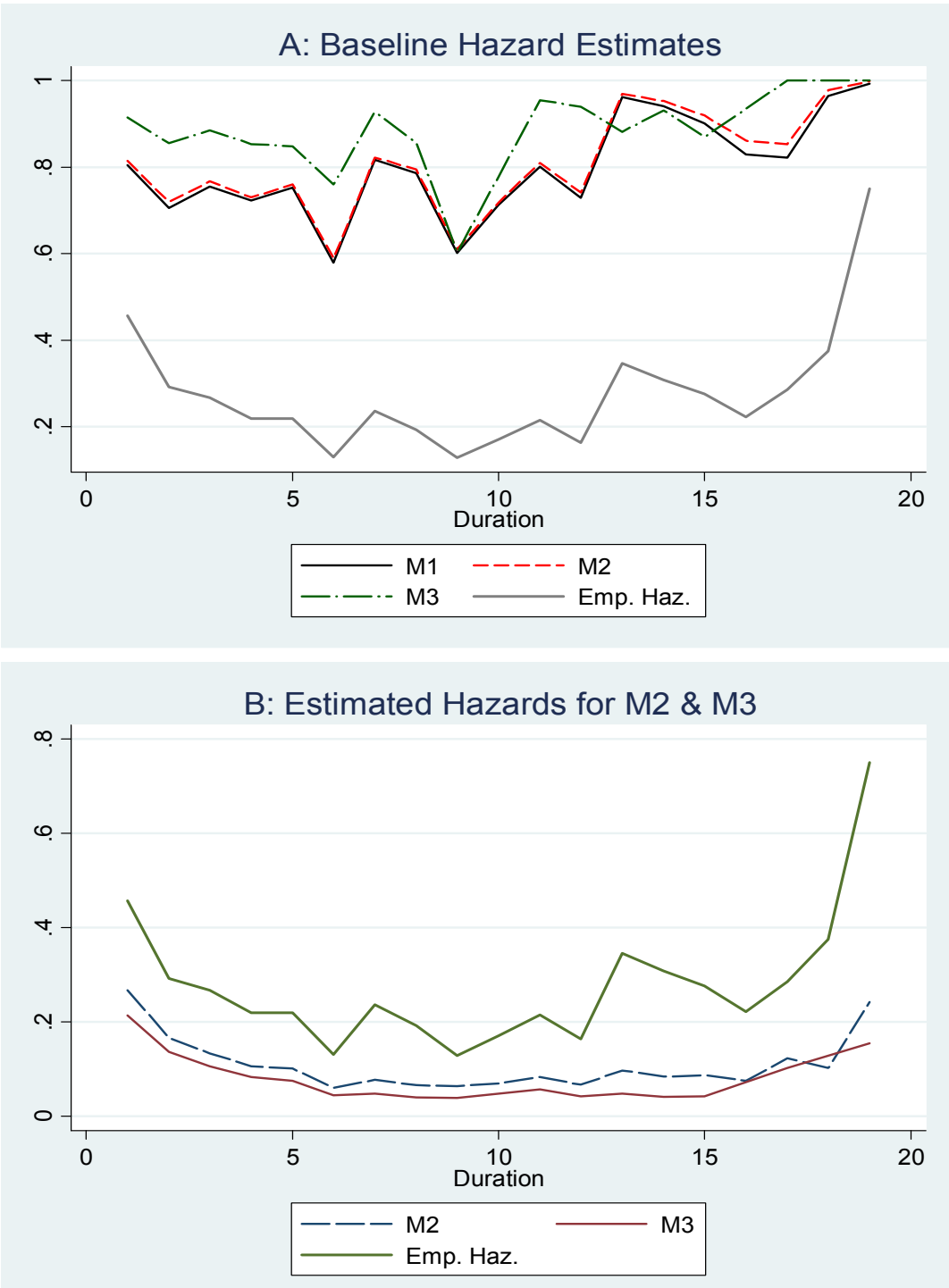

Figure 3.2: (A) Cox Baseline Hazards for Youth Players, (B) Cox Estimated Career Duration Hazards for Youth Players 


\section{Chapter 4 Concluding Remarks}

The thesis examined numerous labour market issues in English and European soccer using two uniquely collected data sets.

Chapter 1 examined the impact of youth training or education on the career and spell duration of soccer players. The results of model estimation suggest that the different training methods employed and ability to assess talent at youth academies play an important role in the longitude of a player's career, while differing spell durations can be relevant in the analysis of behaviour of smaller vs. larger clubs and their ability or will to hold on to their home-grown players. One limitation of the data set and subsequent analysis is that it allows the measuring of local effects of these youth academies, rather than general. Estimating general effects would be an interesting extension, pending data availability. This type of analysis could be possible in different sports in recent times, most likely in North America, considering the larger extent of publicly available data.

Chapter 2 analyses relied on a data set that encompassed a much narrower time frame than the data used in Chapter 1, centered on a major institutional change in European soccer. Players entering on either side of the Bosman ruling were examined and compared, and the results suggest that post-Bosman entrants are generally more productive, which leads to increased career duration in top European leagues. 
The data set is limited in that it account only for new entrants to the English Premier league in that 8-year period, and the effect of the institutional change on the incumbents cannot be assessed. Accounting for incumbents can enrich the analysis, both empirically and theoretically.

Chapter 3 present an analysis that utilizes both data sets in assessing the bottomheaviness of English Premier League clubs and the probability that they would select first-tea players from internal vs. external sources. The results suggest that more bottom-heavy clubs have a higher probability of selecting first-tea players from internal sources, which is in line with relevant theory and studies performed in other industries. Furthermore, additional survival analysis lends support to the results from Chapter 1. Once again, a limitation of the data is that it doesn't account for all youth players under contract at each club, which would allow for a more general and accurate assessment of bottom-heaviness of these clubs and provide more reliable predictions for promoting players from within. This type of data is not yet publicly available, but it is improving in more recent years and could be better represented in North American sports. The question of internal promotion vs. external recruitment definitel deserves more attention in the context of professional sports. 


\section{References}

[1] Abbring, J. H. and G. J. van den Berg (2003): "The non-parametric identificatio of treatment effects in duration models," Econometrica 71, 14911517.

[2] Alvarez, J. et al. (2011): "Impact of Importing Foreign Talent on Performance Levels of Local Co-workers," Labour Economics, 18, 287-296.

[3] Ashworth, J., and Heyndels, B. (2007): "Selection Bias and Peer Effects in Team Sports: the Effect of Age Grouping on Earnings of German Soccer Players," Journal of Sports Economics, 8 (4), 355-377.

[4] Atkinson, S., and Tschirhart, J. (1986): "Flexible Modeling of Time to Failure in Risky Careers," Review of Economics and Statistics, November, 558566.

[5] Battu, H., Belfield C.R., Sloane, P.J. (2003): "Human capital spill-overs within the workplace," Oxford Bulletin of Economics and Statistics, 65 (5), 575-594.

[6] Bayo-Moriones, A. and Ortin-Angel, P. (2006): "Internal promotion versus external recruitment: Industrial plants in Spain," Industrial and Labor Relations Review, 59 (3) 451-470.

[7] Berlinschi, R., Schokkaert, J., and Swinnen, J. (2013). "When drains and gains coincide: Migration and international football performance," Labour Economics, 21, 1-14.

[8] Binder, J., Findlay, M. (2012): "The Effects of the Bosman Ruling on National and Club Teams in Europe," The Journal of Sports Economics, 13(2), 107-129.

[9] Bloom, S. (2012): "Modern Regression Discontinuity Analysis," Journal of Research on Educational Effectiveness, 5(1), 43-82.

[10] DeVaro, J. and Morita, H. (2013): "Internal Promotion and External Recruitment: A Theoretical and Empirical Analysis," Journal of Labor Economics, $31(2), 227-269$. 
[11] Dufour, J.-M., and J. Jasiak (2001): "Finite Sample Limited Information Inference Methods for Structural Equations and Models with Generated Regressors," International Economic Review, 42(3), 815-43.

[12] Elbers, C., and Ridder, G. (1982): "True or Spurious Duration Dependence: The Identifiabilit of the Proportional Hazard Model," Review of Economic Studies, 49, 402-409.

[13] European Club Association (2012): "Report on Youth Academies in Europe," www.ecaeurope.com.

[14] Ferris, S. and Voia, M.C. (2013): "Do business cycle peaks predict election calls in Canada?," European Journal of Political Economy, 29, 109-118.

[15] Franck, H., and Cook, J. (1995): "The Winner-Take-All Society," New York: Free Press.

[16] Frick, B. (2009): "Globalization and factor mobility: the impact of the "Bosman Ruling on player migration in professional soccer," Journal of Sports Economics, 10, 88-106.

[17] Frick, B., Pietzner, G., and Prinz, J. (2007): "Career Duration in a Competitive Environment: The Labor Market for Soccer Players in Germany," Eastern Economic Journal, 33(3).

[18] Fu, Q., Lu, J. (2012): "Micro foundations of multi-prize lottery contests: A perspective of noisy performance ranking," Social Choice and Welfare, 38, 497-517.

[19] Geyer, H. (2010): "Quit Behavior of Professional Tennis Players," Journal of Sports Economics, 11(1), 89-99.

[20] Goddard, J. and Wilson, J.O.S. (2009): Racial discrimination in English professional football: evidence from an empirical analysis of players' career progression, Cambridge Journal of Economics, vol. 33, 295-316.

[21] Gramsch, P.M., and Therneau, T.M. (1994): "Proportional Hazards Tests and Diagnostics Based on Weighted Residuals," Biometrika, 81, 515-526.

[22] Gramsch, P.M., and Therneau, T.M. (2000): "Modeling Survival Data: Extending the Cox Model," New York, Springer-Verlag. 
[23] Groothuis, P.A., and Hill, J.R. (2004): "Exit Discrimination in the NBA: A Duration Analysis of Career Length," Economic Inquiry, 341-349.

[24] Gutierrez, R.G.(2002): "Parametric frailty and shared frailty survival models," The Stata Journal, (2), 22-44.

[25] Harvey, A. (1976): "Estimating Regression Models with Multiplicative Heteroscedasticity," Econometrica, 44 (3), 461-465.

[26] Hastie, T.J., and Tibishirani, R.J. (1990): "Generalized Additive Models," Chapman and Hall, London.

[27] Hausman, J. A., and D. L. McFadden, (1984): "Specificatio tests for the multinomial logit model," Econometrica 52, 1219-1240.

[28] Heckman, J.J., and Singer, B. (1982): "The Identificatio Problem in Econometric Models for Duration Data," Advances in Econometrics, edited by W. Hildenbrand. Cambridge University Press.

[29] Hillman, A., and Riley, J. (1989): "Politically Contestable Rents and Transfers," Economics and Politics, 1, 17-39.

[30] Huynh, K. P.,Petrunia, R.J. and Voia, M.C. (2010): “The Impact of Initial Financial State on Firm Duration Across Entry Cohorts ", Journal of Industrial Economics, Vol.LVIII, no.3.

[31] Imbens, G., and Kalyanaraman K. (2009): "Optimal Bandwidth Choice for the Regression Discontinuity Estimator." NBER WP 14726.

[32] Jenkins, S.P. (2008): "Survival Analysis," Unpublished Manuscript, Institute for Social and Economic Research, University of Essex, Colchester.

[33] Keele, L. (2010): "Proportionality Difficult Testing for Nonproportional Hazards in Cox Models," Political Analysis, 18, 189-205.

[34] Konrad, K. (2007): "Strategy in Contests - An Introduction," Social Science Research Center Berlin.

[35] Konrad, K. (2009): "Strategy and Dynamics in Contests," Oxford University Press, Oxford. 
[36] Lazear, E. P., Rosen, S. (1981): "Rank-order Tournaments as Optimum Labor Contracts," Journal of Political Economy, 89, 841-864.

[37] Lee, D., and Lemieux, T. (2010): "Regression Discontinuity Designs in Economics," Journal of Economic Literature, 48, 281-355.

[38] Milanovic, B. (2005): "Globalization and goals: does soccer show the way?," Review of International Political Economy 12 (5), 829-850.

[39] Noll, R.G (2002): "The Economics of Promotion and Relegation in Sports Leagues: The Case of English Football," The Journal of Sports Economics, May, 169-203.

[40] Okhusa, Y. (2001): "An Empirical Examination of Quit Behavior of Professional Baseball Players in Japan," The Journal of Sports Economics, May, 80-88.

[41] Oyer, P. (2007): "Is there and Insider Advantage in getting Tenure?," American Economic Review, 97 (2), 501-505.

[42] Radoman, M., and Voia, M.: "Youth Training Programs and their Impact on Career and Spell Duration of Professional Soccer Players," Labour: Review of Labour Economics and Industrial Relations, Forthcoming 2015.

[43] Rogers, W. H. (1993): "Comparison of nbreg and glm for negative binomial," Stata Technical Bulletin 16, 7, Reprinted in Stata Technical Bulletin Reprints, 3, 82-84.

[44] Rosen, S. (1985): "Prizes and Incentives in Elimination Tournaments," National Bureau of Economic Research, working paper 1668, Cambridge.

[45] Royston, P., and Sauerbrei W. (2007): "Multivariable Modeling with Cubic Regression Splines: A Principled Approach," The Stata Journal, 7(1), 45-70.

[46] Ryvkin, D. (2009): "Tournaments of Weakly Heterogenous Players," Journal of Public Economic Theory, 11(5), 819-855.

[47] Ryvkin, D. (2013): "Heterogeneity of Players and Aggregate Effort in Contests," Journal of Economics and Management Strategy, 22(4), 728-743. 
[48] Spurr, S., and Barber, W. (1994): "The Effect of productivity on a Worker's Career: Evidence from Minor League Baseball," Industrial and Labor Relations Review, July, 692-708.

[49] Stein, W. (2002): "Asymmetric Rent-Seeking With More than Two Contestants," Public Choice, 113, 325-336.

[50] Szymanski, S. (2003): "The Economic Design of Sporting Contests," Journal of Economic Literature, 41(4), 1137-1187.

[51] Tollison, R. (2012): "The Economic Theory of Rent Seeking," Public Choice, $152,73-82$.

[52] Vijverberg, W. (2011): "Testing for IIA with the Hausman-McFadden Test," CUNY Graduate Center and IZA, Discussion Paper No. 5826.

[53] Vuong, Q. (1989): "Likelihood Ratio Tests for Model Selection and NonNested Hypotheses," Econometrica, 57, 307-333.

[54] Waldman, M. (2013): "Classic promotion tournaments versus market-based tournaments," International Journal of Industrial Organization, 31, 198210.

[55] Waldman, M. (2012): "Theory and evidence in internal labor markets," The Handbook of Organizational Economics, Princeton University Press.

[56] Wilde, J. (2000): "Identificatio of multiple equation probit models with endogenous dummy regressors," Economics Letters, 69, 309-312.

[57] Williams, R. (2009): "Using Heterogeneous Choice Models to Compare Logit and Probit Coefficient Across Groups," Sociological Methods and Research, 37 (4), 531-559.

[58] Zorn, C.J. (2000): "Modeling Duration Dependence," Political Analysis, 8, 367-380. 